\title{
ASYMPTOTICS IN SMALL TIME FOR THE DENSITY OF A STOCHASTIC DIFFERENTIAL EQUATION DRIVEN BY A STABLE LÉVY PROCESS
}

\author{
Emmanuelle Clément ${ }^{1, *}$, Arnaud Gloter ${ }^{2}$ and Huong Nguyen ${ }^{3}$
}

\begin{abstract}
This work focuses on the asymptotic behavior of the density in small time of a stochastic differential equation driven by a truncated $\alpha$-stable process with index $\alpha \in(0,2)$. We assume that the process depends on a parameter $\beta=(\theta, \sigma)^{T}$ and we study the sensitivity of the density with respect to this parameter. This extends the results of [E. Clément and A. Gloter, Local asymptotic mixed normality property for discretely observed stochastic dierential equations driven by stable Lévy processes. Stochastic Process. Appl. 125 (2015) 2316-2352.] which was restricted to the index $\alpha \in(1,2)$ and considered only the sensitivity with respect to the drift coefficient. By using Malliavin calculus, we obtain the representation of the density and its derivative as an expectation and a conditional expectation. This permits to analyze the asymptotic behavior in small time of the density, using the time rescaling property of the stable process.
\end{abstract}

Mathematics Subject Classification. 60G51, 60G52, 60H07, 60H20, 60H10, 60J75.

Received December 12, 2016. Accepted March 5, 2018.

\section{INTRODUCTION}

We consider the following stochastic differential equation (SDE)

$$
X_{t}^{\beta}=x_{0}+\int_{0}^{t} b\left(X_{s}^{\beta}, \theta\right) \mathrm{d} s+\sigma L_{t}
$$

for $t \in[0,1]$, where $\left(L_{t}\right)_{t \in[0,1]}$ is a truncated $\alpha$-stable process with exponent $0<\alpha<2$ and our aim is to study the asymptotic behavior, in small time, of the density of $\left(X_{t}^{\beta}\right)$, the solution of $(1.1)$, as well as its derivative with respect to the parameter $\beta=(\theta, \sigma)^{T}$. This problem plays an important role in asymptotic statistics based on high frequency observations. Indeed, considering the estimation of $\beta$ from the discrete time observations $\left(X_{i / n}^{\beta}\right)_{0 \leq i \leq n}$, and denoting by $p_{1 / n}^{\beta}(x, y)$ the transition density of the discrete time process, the estimation rate of the parameter $\beta$ strongly relies on the asymptotic behavior of the derivative $\nabla_{\beta} p_{1 / n}^{\beta}(x, y)$, as $n$ goes to

Keywords and phrases: Lévy process, density in small time, stable process, Malliavin calculus for jump processes.

${ }^{1}$ Laboratoire MICS, Fédération de Mathématiques FR 3487, Université Paris-Saclay, CentraleSupélec, 91190 Gif-sur-Yvette, France.

${ }^{2}$ Université d'Évry Val d'Essonne, Laboratoire de Mathématiques et Modélisation d'Evry, UMR 8071, 23 Boulevard de France, 91037 Évry Cedex, France.

${ }^{3}$ Université Paris-Est, LAMA, UMR 8050, UPEMLV, CNRS, UPEC, 77454 Marne-la-Vallée, France.

* Corresponding author: emmanuelle.clement@u-pem.fr 
infinity. Based on the results established in the present paper, we derive, in [6], an asymptotic expansion of the $\log$-likelihood ratio and we prove the LAMN property for the parameter $\beta$.

In the last decades, a large literature has been devoted to the existence and regularity of the density to the solution $\left(X_{t}\right)_{t}$, for $t>0$, of a general stochastic equation driven by pure jump Lévy processes. We can mention the works of Bichteler et al. [2], Picard [14], Denis [8], Ishikawa-Kunita [10], Fournier-Printems [9] and more recently the works of Debussche-Fournier [7] and Kulik [13], under Hölder continuity assumptions on the coefficients of the equation and assuming that the equation is driven by an $\alpha$-stable process.

In this paper, the main contributions are obtained by using the Malliavin calculus for jump processes developed by Bichteler et al. [2] and adapted to the particular case of equation (1.1) by Clément and Gloter [5]. Although it requires some strong derivability assumptions on the coefficients of the equation, it leads to some explicit representation formulas for the density and its derivative (see also Ivanenko-Kulik [11]). Let us mention that alternative representations for the density can be obtained by other methods, for example the method proposed by Bouleau-Denis [3] based on Dirichlet forms or the parametrix method used by Kulik [13].

To study the asymptotic behavior of the transition density of $X_{t}^{\beta}$ and its derivative, in small time, we establish some representation formulas. This extends the results of Clément and Gloter [5] where only the derivative with respect to the drift parameter $\theta$ was considered, with the restriction $\alpha>1$. These representation formulas involve some Malliavin weights whose expressions are given explicitly. This permits first to identify in the Malliavin weights a main part and a negligible part in small time asymptotics and then to derive the asymptotics for the density stated in Theorems 2.2 and 2.5. In contrast to [5], the exposition now involves the solution of the ordinary differential equation defined by the deterministic part of (1.1). Moreover, the study of each terms appearing in the Malliavin weights is complicated by the non integrability of the $\alpha$-stable process as $\alpha \leq 1$.

The present paper is organized as follows. Section 2 contains the main results (Thms. 2.2 and 2.5). Section 3 presents the methodology consisting first in a representation of the density by Malliavin calculus where the Malliavin weights can be decomposed into a main part and a negligible part and then in the study of their asymptotic behavior. We prove our main results in Sections 4 and 5. Finally, in Section A, we recall the Malliavin integration by parts setting developed by [2] and used in [5], and give some representations of the transition density, its derivative, as well as its logarithm derivative. We also explicit the iterated Malliavin weights appearing in the expression of the derivative of the density.

\section{Asymptotics FOR THE DENSITy AND ITS DERIVATIVE}

We consider the process $\left(X_{t}^{\beta}\right)_{t \in[0,1]}$ solution to the stochastic equation (1.1) where $\left(L_{t}\right)_{t \in[0,1]}$ is a pure jump Lévy process defined on a filtered probability space $\left(\Omega, \mathcal{G},\left(\mathcal{G}_{t}\right)_{t \in[0,1]}, \mathbb{P}\right), b$ is a real valued function and the parameter $\beta=(\theta, \sigma)^{T}$ belongs to $\mathbb{R} \times(0, \infty)$. We assume that the following assumptions are fulfilled.

$\mathbf{H}_{1}$ : (a) The function $b$ has bounded derivatives up to order five with respect to both variables.

$\left(b_{i}\right)$ The Lévy process $\left(L_{t}\right)_{t \in[0,1]}$ is given by $L_{t}=\int_{0}^{t} \int_{[-1,1]} z\{\bar{\mu}(\mathrm{d} s, \mathrm{~d} z)-\bar{v}(\mathrm{~d} s, \mathrm{~d} z)\}+\int_{0}^{t} \int_{[-1,1]^{c}} z \bar{\mu}(\mathrm{d} s, \mathrm{~d} z)$ where $\bar{\mu}$ is a Poisson random measure, with compensator $\bar{v}(\mathrm{~d} t, \mathrm{~d} z)=\mathrm{d} t \times F(z) \mathrm{d} z$ where $F(z)$ is given on $\mathbb{R}^{*}$ by $F(z)=\frac{1}{|z|^{\alpha+1}} \tau(z), \alpha \in(0,2)$. Moreover, we assume that $\tau$ is a non negative smooth function equal to 1 on $[-1,1]$, vanishing on $[-2,2]^{c}$ such that $0 \leq \tau \leq 1$.

$\left(b_{i i}\right)$ We assume that $\forall p \geq 1, \int_{\mathbb{R}}\left|\frac{\tau^{\prime}(u)}{\tau(u)}\right|^{p} \tau(u) \mathrm{d} u<\infty, \int_{\mathbb{R}}\left|\frac{\tau^{\prime \prime}(u)}{\tau(u)}\right|^{p} \tau(u) \mathrm{d} u<\infty$.

Under these assumptions, $X_{t}^{\beta}$ admits a smooth density for $t>0$ (see Sect. A), and we denote by $p_{t}^{\beta}(x, y)$ the transition density of the Markov process $\left(X_{t}^{\beta}\right)$.

Throughout the paper, we will use the following notation. For a vector $h \in \mathbb{R}^{2}, h^{T}$ denotes the transpose of $h$, and $|h|$ denotes the euclidean norm. For a function $f$ defined on $\mathbb{R} \times \mathbb{R}^{2}$ depending on both variables $(x, \beta)$, here $\beta=(\theta, \sigma)^{T} \in \mathbb{R} \times(0,+\infty)$, we denote by $f^{\prime}$ the derivative of $f$ with respect to the variable $x$, by $\partial_{\theta} f$ the derivative of $f$ with respect to the parameter $\theta$, by $\partial_{\sigma} f$ the derivative of $f$ with respect to the parameter $\sigma$, and $\nabla_{\beta} f=\left(\begin{array}{c}\partial_{\theta} f \\ \partial_{\sigma} f\end{array}\right)$. 
The regularity assumption $\mathbf{H}_{1}(\mathrm{a})$ on the drift coefficient $b$ is a sufficient condition to obtain the representations of the density and its derivative. This assumption could be weakened but our methodology is based on the Malliavin calculus developed in [2] that requires strong regularity assumptions on the coefficients. From Theorem 10-3 in [2], the computation of the Malliavin operators for a stochastic differential equation needs coefficients with derivatives up to order three. As we iterate the Malliavin operators we need derivatives up to order five (see Lem. A.6). Note that we relax the boundedness assumption on $b$ assumed in [5].

Remark 2.1. The assumptions on the Lévy measure are restrictive and one may expect that our results hold with a more general Lévy measure $F(z)=\frac{1}{|z|^{\alpha+1}} g(z)$, where $g$ satisfies $\left(b_{i i}\right)$ and $g(0)=1$. However in our approach the integrability assumptions for the tails of the Lévy process are crucial to ensure that our process belongs to the Malliavin space. Moreover, the exact $\alpha$-stable behavior of the Lévy measure around zero is also largely used (see Lem. 3.1) to study the Malliavin weights asymptotics. The truncation function $\tau$ ensures both the integrability of $\left|L_{t}\right|^{p}, \forall p \geq 1$, and the exact $\alpha$-stable behavior around zero $(\tau=1)$. It permits the careful study of each Malliavin terms appearing in the representation formulas (3.12) and (5.1). All these terms are not yet being in control without these restrictions on the Lévy measure.

Our aim is to study the asymptotic behavior of $p_{\frac{1}{n}}^{\beta}\left(x_{0}, u\right)$ (the density of $\left.X_{\frac{1}{n}}^{\beta}\right)$ and its derivative with respect to the parameter $\beta$. To this end, we introduce the solution to the ordinary differential equation

$$
\varsigma_{t}^{n, \theta, x_{0}}=x_{0}+\frac{1}{n} \int_{0}^{t} b\left(\varsigma_{s}^{n, \theta, x_{0}}, \theta\right) \mathrm{d} s \quad t \in[0,1]
$$

Heuristically, $n^{1 / \alpha}\left(X_{1 / n}^{\beta}-\varsigma_{1}^{n, \theta, x_{0}}\right)$ is close to $\sigma n^{1 / \alpha} L_{1 / n}$ and from assumption $\mathbf{H}_{1}\left(b_{i}\right)$, the rescaled process $\left(n^{1 / \alpha} L_{t / n}\right)_{t \in[0,1]}$ converges in distribution to an $\alpha$-stable process $\left(L_{t}^{\alpha}\right)_{t \in[0,1]}$ (see Sect. 3.1). Our first result shows that the density of $\frac{n^{1 / \alpha}}{\sigma}\left(X_{1 / n}^{\beta}-\varsigma_{1}^{n, \theta, x_{0}}\right)$ converges to the density of $L_{1}^{\alpha}$, as $n$ goes to infinity.

In what follows, we denote by $\varphi_{\alpha}$ the density of $L_{1}^{\alpha}$ where $\left(L_{t}^{\alpha}\right)$ is an $\alpha$-stable process with Lévy measure $v(\mathrm{~d} z)=\frac{1}{|z|^{\alpha+1}} 1_{z \neq 0} \mathrm{~d} z$.

With these notations, we can state our main results. In view of statistical applications, we need some uniformity with respect to the parameter around the true value $\beta=(\theta, \sigma)^{T}$ and consequently we study the asymptotic behavior of $p_{\frac{1}{n}}^{\beta_{n}}$ where $\left(\beta_{n}\right)_{n \geq 1}=\left(\left(\theta_{n}, \sigma_{n}\right)^{T}\right)_{n \geq 1}$ is a sequence converging to $\beta$.

Theorem 2.2. Let $\left(\varsigma_{t}^{n, \theta, x_{0}}\right)$ be the solution to the ordinary differential equation (2.1) and let $\left(\beta_{n}\right)_{n \geq 1}$ be a sequence such that $\beta_{n} \stackrel{n \rightarrow \infty}{\longrightarrow} \beta$. For all $\left(x_{0}, u\right) \in \mathbb{R}^{2}$,

$$
\begin{aligned}
& \text { 1. } \frac{\sigma_{n}}{n^{1 / \alpha}} p_{\frac{1}{n}}^{\beta_{n}}\left(x_{0}, \frac{u \sigma_{n}}{n^{1 / \alpha}}+\varsigma_{1}^{n, \theta_{n}, x_{0}}\right) \stackrel{n \rightarrow \infty}{\longrightarrow} \varphi_{\alpha}(u), \\
& \text { 2. } \sup _{u \in \mathbb{R}} \sup _{n} \frac{\sigma_{n}}{n^{1 / \alpha}} p_{\frac{1}{n}}^{\beta_{n}}\left(x_{0}, \frac{u \sigma_{n}}{n^{1 / \alpha}}+\varsigma_{1}^{n, \theta_{n}, x_{0}}\right)<\infty,
\end{aligned}
$$

where $\varphi_{\alpha}$ is the density of $L_{1}^{\alpha}$.

If the solution to the ordinary equation (1.1) is not given explicitly, we can approximate it by a numerical scheme. The previous convergence will be preserved if the order of the numerical scheme is sufficiently high. This is explained in the next remark.

Remark 2.3. If we assume that the function $b$ is of class $\mathcal{C}^{1+k}$ with respect to $x(k \geq 0)$ and setting $A(f)=b f^{\prime}$ (and $\left.A^{0}(f)=f\right)$ such that $f\left(\varsigma_{t}^{n, \theta, x_{0}}, \theta\right)=f\left(\varsigma_{0}^{n, \theta, x_{0}}, \theta\right)+\int_{0}^{t}(A f)\left(\varsigma_{s}^{n, \theta, x_{0}}, \theta\right) \mathrm{d} s$, we obtain

$$
\begin{aligned}
\varsigma_{t}^{n, \theta, x_{0}} & =x_{0}+\frac{1}{n} \int_{0}^{t} A^{0}(b)\left(\varsigma_{s}^{n, \theta, x_{0}}, \theta\right) \mathrm{d} s \\
& =x_{0}+\frac{t\left(A^{0} b\right)\left(x_{0}, \theta\right)}{n}+\frac{1}{n} \int_{0}^{t} \int_{0}^{t_{1}} \frac{(A b)}{n}\left(\varsigma_{t_{2}}^{n, \theta, x_{0}}, \theta\right) \mathrm{d} t_{2} \mathrm{~d} t_{1}
\end{aligned}
$$




$$
=\varsigma_{t}^{(k), n, \theta, x_{0}}+\frac{1}{n^{k+1}} \int_{0}^{t} \int_{0}^{t_{1}} \cdots \int_{0}^{t_{k}}\left(A^{k} b\right)\left(\varsigma_{t_{k+1}}^{n, \theta, x_{0}}, \theta\right) \mathrm{d} t_{k+1} \cdots \mathrm{d} t_{t_{1}}
$$

with $\varsigma_{t}^{(k), n, \theta, x_{0}}=x_{0}+\frac{t\left(A^{0} b\right)\left(x_{0}, \theta\right)}{n}+\frac{t^{2}(A b)\left(x_{0}, \theta\right)}{2 n^{2}}+\cdots+\frac{t^{k}\left(A^{k-1} b\right)\left(x_{0}, \theta\right)}{k ! n^{k}}$, for $k \geq 1$, and $\varsigma_{t}^{(0), n, \theta, x_{0}}=x_{0}$.

Assuming moreover that the function $b$ has bounded derivatives, we deduce that $\left|\varsigma_{t}^{n, \theta, x_{0}}-\varsigma_{t}^{(k), n, \theta, x_{0}}\right| \leq \frac{C}{n^{k+1}}$.

Then from the proof of Theorem 2.2, if $n^{1 / \alpha} / n^{k+1}$ goes to zero, we can replace $\varsigma_{1}^{n, \theta_{n}, x_{0}}$ by $\varsigma_{1}^{(k), n, \theta_{n}, x_{0}}$ in the statement of the theorem and we obtain

$$
\frac{\sigma_{n}}{n^{1 / \alpha}} p_{\frac{1}{n}}^{\beta_{n}}\left(x_{0}, \frac{u \sigma_{n}}{n^{1 / \alpha}}+\varsigma_{1}^{(k), n, \theta_{n}, x_{0}}\right) \stackrel{n \rightarrow \infty}{\longrightarrow} \varphi_{\alpha}(u), \quad \text { if } \quad k>\frac{1}{\alpha}-1 .
$$

In particular, if $\alpha>1$, the choice $\varsigma_{1}^{(0), n, \theta_{n}, x_{0}}=x_{0}$ is convenient as established in [5].

Remark 2.4. The results of Theorem 2.2 have been obtained by Kulik [13], using the parametrix method.

The next result gives the asymptotic behavior of the derivatives of the density with respect to the parameters $\theta$ and $\sigma$.

Theorem 2.5. Let $\left(\beta_{n}\right)_{n \geq 1}$ be a sequence such that $\beta_{n} \stackrel{n \rightarrow \infty}{\longrightarrow} \beta$. For all $\left(x_{0}, u\right) \in \mathbb{R}^{2}$,

i) $\frac{\sigma_{n}^{2}}{n^{\frac{2}{\alpha}-1}} \partial_{\theta} p_{\frac{1}{n}}^{\beta_{n}}\left(x_{0}, \frac{u \sigma_{n}}{n^{1 / \alpha}}+\varsigma_{1}^{n, \theta_{n}, x_{0}}\right) \stackrel{n \rightarrow \infty}{\longrightarrow}-\partial_{\theta} b\left(x_{0}, \theta\right) \times \varphi_{\alpha}^{\prime}(u)$,

$$
\frac{\sigma_{n}^{2}}{n^{1 / \alpha}} \partial_{\sigma} p_{\frac{1}{n}}^{\beta_{n}}\left(x_{0}, \frac{u \sigma_{n}}{n^{1 / \alpha}}+\varsigma_{1}^{n, \theta_{n}, x_{0}}\right) \stackrel{n \rightarrow \infty}{\longrightarrow}-\varphi_{\alpha}(u)-u \varphi_{\alpha}^{\prime}(u)
$$

ii) $\sup _{u \in \mathbb{R}} \sup _{n}\left|\frac{\sigma_{n}^{2}}{n^{\frac{2}{\alpha}-1}} \partial_{\theta} p_{\frac{1}{n}}^{\beta_{n}}\left(x_{0}, \frac{u \sigma_{n}}{n^{1 / \alpha}}+\varsigma_{1}^{n, \theta_{n}, x_{0}}\right)\right|<\infty$,

$$
\sup _{u \in \mathbb{R}} \sup _{n}\left|\frac{\sigma_{n}^{2}}{n^{1 / \alpha}} \partial_{\sigma} p_{\frac{1}{n}}^{\beta_{n}}\left(x_{0}, \frac{u \sigma_{n}}{n^{1 / \alpha}}+\varsigma_{1}^{n, \theta_{n}, x_{0}}\right)\right|<\infty .
$$

Considering the statistical experiment $\left(\mathbb{R}^{n}, \mathcal{B}_{n}, \mathbb{P}_{n}^{\beta}\right)$ corresponding to the observation of $\left(X_{i / n}^{\beta}\right)_{1 \leq i \leq n}$, Theorems 2.2 and 2.5 permit to prove in [6] the $L^{2}$-regularity property of the transition density $p_{1 / n}^{\beta}(x, y)$ :

$$
\sum_{i=1}^{n} \mathbb{E}\left[\int_{\mathbb{R}}\left\{p_{\frac{1}{n}}^{\beta+r_{n} h}\left(X_{\frac{i-1}{n}}^{\beta}, y\right)^{1 / 2}-p_{\frac{1}{n}}^{\beta}\left(X_{\frac{i-1}{n}}^{\beta}, y\right)^{1 / 2}-\frac{1}{2} h^{T} r_{n} \frac{\nabla_{\beta} p_{\frac{1}{n}}^{\beta}\left(X_{\frac{i-1}{n}}^{\beta}, y\right)}{\left(p_{\frac{1}{n}}^{\beta}\right)^{1 / 2}\left(X_{\frac{i-1}{n}}^{\beta}, y\right)}\right\}^{2} \mathrm{~d} y\right] \stackrel{n \rightarrow \infty}{\longrightarrow} 0,
$$

with rate $r_{n}=\left(\begin{array}{cc}n^{\frac{1}{2}-\frac{1}{\alpha}} & 0 \\ 0 & n^{-\frac{1}{2}}\end{array}\right)$. In this application, the sequence $\left(\beta_{n}=\left(\theta_{n}, \sigma_{n}\right)^{T}\right)$ is $\left(\beta+r_{n} h\right)$. The $L^{2}$ regularity property (related to the $L^{2}$-differentiability of $\beta \mapsto\left(p_{\frac{1}{n}}^{\beta}\right)^{1 / 2}$ ) is the first step to obtain an asymptotic expansion of the $\log$-likelihood ratio $\log \frac{d \mathbb{P}_{n}^{\beta+r_{n} h}}{d \mathbb{P}_{n}^{\beta}}\left(X_{1 / n}^{\beta}, \ldots, X_{1}^{\beta}\right)$ (see Thm. 2.1 in [6]) and to deduce the Local Asymptotic Mixed Normality property (see Jeganathan [12]). We proved that the LAMN property holds (Cor. 2.4 in [6]) for the parameter $\beta$ with rate $r_{n}$ and information matrix $\mathcal{I}=\left(\begin{array}{cc}\mathcal{I}_{11} & 0 \\ 0 & \mathcal{I}_{22}\end{array}\right)$ where $\mathcal{I}_{11}=\frac{1}{\sigma^{2}} \int_{0}^{1} \partial_{\theta} b\left(X_{s}^{\beta}, \theta\right)^{2} \mathrm{~d} s \int_{\mathbb{R}} \frac{\varphi_{\alpha}^{\prime}(u)^{2}}{\varphi_{\alpha}(u)} \mathrm{d} u$ and $\mathcal{I}_{22}=\frac{1}{\sigma^{2}} \int_{\mathbb{R}} \frac{\left(\varphi_{\alpha}(u)+u \varphi_{\alpha}^{\prime}(u)\right)^{2}}{\varphi_{\alpha}(u)} \mathrm{d} u$. As a consequence, we deduce that the estimation rate for $\theta$ is $n^{\frac{1}{2}-\frac{1}{\alpha}}$ and that the estimation rate for $\sigma$ is the usual one $n^{-\frac{1}{2}}$. Moreover, the best asymptotic variance of any regular estimator of $\beta$ (that converges in distribution with rate $r_{n}$ ) is the inverse of the information matrix $\mathcal{I}$. 
The proofs of Theorems 2.2 and 2.5 are based on the representations of the density and its derivative obtained by using Malliavin calculus and on the study of the asymptotic behavior of the Malliavin weights. This is given in the next sections.

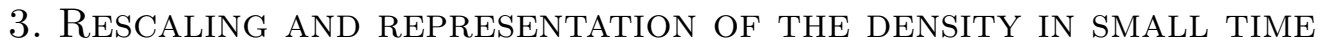

In this section, we give a representation of the density and identify in this representation the main terms and the remainder terms. This decomposition is a key step for the convergence study and is mainly based on the rescaling described in the next subsection.

\subsection{Rescaling}

We can observe that the process $\left(n^{1 / \alpha} L_{t / n}\right)$ equals in law to a centered Lévy process with Lévy measure

$$
F_{n}(z)=\frac{1}{|z|^{1+\alpha}} \tau\left(\frac{z}{n^{1 / \alpha}}\right) .
$$

As mentioned previously, this clearly suggests that when $n$ grows, the process $\left(n^{1 / \alpha} L_{t / n}\right)$ converges to an $\alpha$ stable process. In the sequel, it will be convenient to construct a family of Lévy processes $\left(L_{t}^{n}\right)_{n \geq 1}$ with the same law as $\left(n^{1 / \alpha} L_{t / n}\right)$, on a common probability space where the limiting $\alpha$-stable process exists as well, and where the convergence holds true in a path-wise sense, as done in [5].

Let us consider $\mu^{e}(\mathrm{~d} t, \mathrm{~d} z, \mathrm{~d} u)$ a Poisson measure on $[0, \infty) \times \mathbb{R}^{*} \times[0,1]$ with compensating measure $v^{e}(\mathrm{~d} t, \mathrm{~d} z, \mathrm{~d} u)=\mathrm{d} t \frac{\mathrm{d} z}{|z|^{1+\alpha}} \mathrm{d} u$ and we denote by $\tilde{\mu}^{e}(\mathrm{~d} t, \mathrm{~d} z, \mathrm{~d} u)=\mu^{e}(\mathrm{~d} t, \mathrm{~d} z, \mathrm{~d} u)-v^{e}(\mathrm{~d} t, \mathrm{~d} z, \mathrm{~d} u)$ the compensated Poisson random measure. This measure corresponds to the jump measure of an $\alpha$-stable process, where each jump is marked with an uniform variable on $[0,1]$.

We define the Poisson measures $\mu^{(n)}$, for all $n \geq 1$, and $\mu$ by setting :

$$
\begin{aligned}
& \left.\forall A \subset[0, \infty) \times \mathbb{R}, \quad \mu^{(n)}(A)=\int_{[0, \infty)} \int_{\mathbb{R}} \int_{[0,1]} 1_{A}(t, z) 1_{\{u \leq \tau}\left(\frac{z}{n^{1 / \alpha}}\right)\right\}^{\mu^{e}(\mathrm{~d} t, \mathrm{~d} z, \mathrm{~d} u),} \\
& \forall A \subset[0, \infty) \times \mathbb{R}, \quad \mu(A)=\int_{[0, \infty)} \int_{\mathbb{R}} \int_{[0,1]} 1_{A}(t, z) \mu^{e}(\mathrm{~d} t, \mathrm{~d} z, \mathrm{~d} u) .
\end{aligned}
$$

By simple computation, one can check that the compensator of the measure $\mu^{(n)}(\mathrm{d} t, \mathrm{~d} z)$ is $v^{(n)}(\mathrm{d} t, \mathrm{~d} z)=$ $\mathrm{d} t \times \tau\left(\frac{z}{n^{1 / \alpha}}\right) \frac{\mathrm{d} z}{|z|^{1+\alpha}}=\mathrm{d} t \times F_{n}(z) \mathrm{d} z$ and the compensator of $\mu(\mathrm{d} t, \mathrm{~d} z)$ is $v(\mathrm{~d} t, \mathrm{~d} z)=\mathrm{d} t \times \frac{\mathrm{d} z}{|z|^{1+\alpha}}$. Moreover, we note $\tilde{\mu}^{(n)}(\mathrm{d} t, \mathrm{~d} z)=\mu^{(n)}(\mathrm{d} t, \mathrm{~d} z)-v^{(n)}(\mathrm{d} t, \mathrm{~d} z)$ and $\tilde{\mu}(\mathrm{d} t, \mathrm{~d} z)=\mu(\mathrm{d} t, \mathrm{~d} z)-v(\mathrm{~d} t, \mathrm{~d} z)$ the compensated Poisson random measures. Remark that since $\tau(z)=1$ for $|z| \leq 1$, the measures $\mu^{(n)}(\mathrm{d} t, \mathrm{~d} z)$ and $\mu(\mathrm{d} t, \mathrm{~d} z)$ coincide on the set $\left\{(t, z)|t \in[0,1]| z \mid, \leq n^{1 / \alpha}\right\}$.

Now we define the stochastic processes associated to these random measures,

$$
\begin{gathered}
L_{t}^{\alpha}=\int_{0}^{t} \int_{[-1,1]} z \tilde{\mu}(\mathrm{d} s, \mathrm{~d} z)+\int_{0}^{t} \int_{[-1,1]^{c}} z \mu(\mathrm{d} s, \mathrm{~d} z) \\
L_{t}^{n}=\int_{0}^{t} \int_{\left[-n^{1 / \alpha}, n^{1 / \alpha}\right]} z \tilde{\mu}^{(n)}(\mathrm{d} s, \mathrm{~d} z)+\int_{0}^{t} \int_{\left[-n^{1 / \alpha}, n^{1 / \alpha}\right]^{c}} z \mu^{(n)}(\mathrm{d} s, \mathrm{~d} z) .
\end{gathered}
$$

By construction, the process $\left(L_{t}^{\alpha}\right)$ is a centered $\alpha$-stable process and the process $\left(L_{t}^{n}\right)$ is equal in law to the process $\left(n^{1 / \alpha} L_{t / n}\right)_{t \in[0,1]}$, since they are based on random measures with the same compensator. Remark that the jumps of $L_{t}^{n}$ with size smaller than $n^{1 / \alpha}$ exactly coincide with the jumps of $L^{\alpha}$ with size smaller than $n^{1 / \alpha}$. On the other hand, the process $L^{n}$ has no jump with a size greater than $2 n^{1 / \alpha}$. 
Using that the measures $\mu$ and $\mu^{(n)}$ coincide on the subsets of $\left\{(t, z) ;|z| \leq n^{1 / \alpha}\right\}$, and the function $\tau\left(\frac{z}{n^{1 / \alpha}}\right) \frac{1}{|z|^{1+\alpha}}=\frac{1}{|z|^{1+\alpha}}$ is symmetric on $|z| \leq n^{1 / \alpha}$, we can rewrite:

$$
L_{t}^{n}=\int_{0}^{t} \int_{[-1,1]} z \tilde{\mu}(\mathrm{d} s, \mathrm{~d} z)+\int_{0}^{t} \int_{1<|z|<n^{1 / \alpha}} z \mu(\mathrm{d} s, \mathrm{~d} z)+\int_{0}^{t} \int_{n^{1 / \alpha} \leq|z| \leq 2 n^{1 / \alpha}} z \mu^{(n)}(\mathrm{d} s, \mathrm{~d} z) .
$$

The following simple lemma gives a connection between $L^{n}$ and the stable process $L^{\alpha}$.

Lemma 3.1. On the event $A_{n}=\left\{\mu\left(\left\{(t, z)|0 \leq t \leq 1| z \mid, \geq n^{1 / \alpha}\right\}\right)=0\right\}$, we have

$$
\mu^{(n)}=\mu, \quad L_{t}^{n}=L_{t}^{\alpha},
$$

and

$$
\mathbb{P}\left(A_{n}\right)=1+O(1 / n)
$$

Furthermore, let $\left(f_{n}\right)_{n \in \mathbb{N}}$ and $f$ be measurable functions from $\Omega \times[0,1] \times \mathbb{R}$ to $\mathbb{R}$ such that there exists $C$ with $\mathbb{P}(C)=1$ and $\forall \omega \in C, \forall s \in[0,1], \forall|z|>1 f_{n}(\omega, s, z) \stackrel{n \rightarrow \infty}{\longrightarrow} f(\omega, s, z)$. Then

$$
\int_{0}^{1} \int_{|z|>1} f_{n}(\omega, s, z) \mu^{(n)}(\mathrm{d} s, \mathrm{~d} z) \underset{\text { a.s. }}{\stackrel{n \rightarrow \infty}{\longrightarrow}} \int_{0}^{1} \int_{|z|>1} f(\omega, s, z) \mu(\mathrm{d} s, \mathrm{~d} z) .
$$

Moreover, we have $\sup _{t \in[0,1]}\left|L_{t}^{n}-L_{t}^{\alpha}\right| \underset{\text { a.s. }}{\stackrel{n \rightarrow \infty}{\longrightarrow}} 0$.

Proof. We know that the measures $\mu^{(n)}$ and $\mu$ coincide on the set $\left\{(s, z)|s \in[0,1]| z \mid, \leq n^{1 / \alpha}\right\}$, and by comparison of the representations (3.2) and (3.4), it is clear that equation (3.5) holds true on the event that the supports of the random measure $\mu$ and $\mu^{(n)}$ do not intersect $\left\{(t, z)|0 \leq t \leq 1| z \mid, \geq n^{1 / \alpha}\right\}$. On the other hand, the support of $\mu^{(n)}$ is included in the support of $\mu$, and thus (3.5) is true on the event $A_{n}=\left\{\mu\left(\left\{(t, z)|0 \leq t \leq 1| z \mid, \geq n^{1 / \alpha}\right\}\right)=0\right\}$. The probability of the latter event is $e^{-2 / \alpha n}$ which converges to 1 at rate $1 / n$ as stated. Then we also get (3.6).

Let $A=\cup_{n=1}^{\infty} A_{n}$, we get that $P(A)=1$ since $A_{n} \subseteq A_{n+1}$ for each $n \in \mathbb{N}$ and (3.6) holds. Thus, for all $\omega \in A \cap C, \exists n_{0}(\omega) \geq 1, \forall n \geq n_{0}(\omega), \mu^{(n)}=\mu$ and $f_{n}(\omega, s, z) \rightarrow f(\omega, s, z) \forall s \in[0,1], \forall|z|>1$. Then we deduce that

$$
\int_{0}^{1} \int_{|z|>1} f_{n}(\omega, s, z) \mu^{(n)}(\mathrm{d} s, \mathrm{~d} z) \underset{\text { a.s. }}{\stackrel{n \rightarrow \infty}{\longrightarrow}} \int_{0}^{1} \int_{|z|>1} f(\omega, s, z) \mu(\mathrm{d} s, \mathrm{~d} z) .
$$

We also get $\sup _{t \in[0,1]}\left|L_{t}^{n}-L_{t}^{\alpha}\right| \stackrel{n \rightarrow \infty}{\longrightarrow} 0$.

\subsection{Representation of the density in small time and first approximation}

We introduce the process $\left(\bar{Y}_{t}^{n, \beta, x_{0}}\right)_{t \in[0,1]}$ given by

$$
\bar{Y}_{t}^{n, \beta, x_{0}}=x_{0}+\frac{1}{n} \int_{0}^{t} b\left(\bar{Y}_{s}^{n, \beta, x_{0}}, \theta\right) \mathrm{d} s+\frac{\sigma}{n^{1 / \alpha}} L_{t}^{n} \quad t \in[0,1]
$$

where $\left(L_{t}^{n}\right)$ is defined by (3.4) and is such that $\frac{1}{n^{1 / \alpha}}\left(L_{t}^{n}\right)$ equals in law to $\left(L_{t / n}\right)$. By construction, the process $\left(X_{\frac{t}{n}}^{\beta}\right)_{t \in[0,1]}$ equals in law $\left(\bar{Y}_{t}^{n, \beta, x_{0}}\right)_{t \in[0,1]}$. Let $q^{n, \beta, x_{0}}$ be the density of $\bar{Y}_{1}^{n, \beta, x_{0}}$ then the connection between the 
densities of $X_{\frac{1}{n}}^{\beta}$ and $\bar{Y}_{1}^{n, \beta, x_{0}}$ is given by

$$
p_{1 / n}^{\beta}\left(x_{0}, x\right)=q^{n, \beta, x_{0}}(x) .
$$

Using the Malliavin calculus for jump processes recalled in Section A, we get a representation of the density of $X_{\frac{1}{n}}^{\beta}$ involving some random variable $\mathcal{H}_{\bar{Y}_{1}^{n, \beta, x_{0}}}(1)$ (Malliavin weight). Moreover, we obtain an asymptotic expansion of this weight that leads to the decomposition of the density into a main part and a remainder part.

To state our next result we define $\left(\epsilon_{s}^{n}\right)_{s \in[0,1]}$ by

$$
\epsilon_{s}^{n}=\exp \left(\frac{1}{n} \int_{0}^{s} b^{\prime}\left(\bar{Y}_{u}^{n, \beta, x_{0}}, \theta\right) \mathrm{d} u\right)
$$

and the function $\rho^{n}$

$$
\rho^{n}(z)= \begin{cases}z^{4} & \text { if }|z|<1 \\ \zeta(z) & \text { if } 1 \leq|z| \leq 2 \\ z^{2} \tau\left(\frac{z}{2 n^{1 / \alpha}}\right) & \text { if }|z|>2\end{cases}
$$

where $\tau$ is defined in assumption $\mathbf{H}_{1}\left(b_{i}\right)$, and $\zeta$ is a non negative function belonging to $\mathbf{C}^{\infty}$ such that $\rho^{n}$ belongs to $\mathbf{C}^{\infty}$. The function $\rho^{n}$ is an auxiliary function related the the Malliavin calculus developed in [2]. In our setting, the above choice (not unique) is convenient (see Rem. 4.1).

Theorem 3.2. Under the assumption $\boldsymbol{H}_{1}$, we have

$$
p_{\frac{1}{n}}^{\beta}\left(x_{0}, u\right)=q^{n, \beta, x_{0}}(u)=\mathbb{E}\left(1_{\left\{\bar{Y}_{1}^{n, \beta, x_{0}} \geq u\right\}} \mathcal{H}_{\bar{Y}_{1}^{n, \beta, x_{0}}}(1)\right),
$$

with

$$
\mathcal{H}_{\bar{Y}_{1}^{n, \beta, x_{0}}}(1)=\frac{1}{\sigma} n^{1 / \alpha}\left[\widehat{\mathcal{H}}_{1, \beta}^{n}(1)+\widehat{\mathcal{H}}_{2, \beta}^{n}(1)\right]+\mathcal{R}_{1, \beta}^{n}(1)+\mathcal{R}_{2, \beta}^{n}(1)+\mathcal{R}_{3, \beta}^{n}(1) .
$$

The main terms $\widehat{\mathcal{H}}_{1, \beta}^{n}(1), \widehat{\mathcal{H}}_{2, \beta}^{n}(1)$ are given by

$$
\begin{aligned}
& \widehat{\mathcal{H}}_{1, \beta}^{n}(1)=\left[\frac{\int_{0}^{1} \int_{\mathbb{R}}\left(\epsilon_{s}^{n}\right)^{-3} \rho^{n}(z)\left(\rho^{n}\right)^{\prime}(z) \mu^{(n)}(\mathrm{d} s, \mathrm{~d} z)}{\epsilon_{1}^{n}\left[\int_{0}^{1} \int_{\mathbb{R}}\left(\epsilon_{s}^{n}\right)^{-2} \rho^{n}(z) \mu^{(n)}(\mathrm{d} s, \mathrm{~d} z)\right]^{2}}\right], \\
& \widehat{\mathcal{H}}_{2, \beta}^{n}(1)=\left[-\frac{\int_{0}^{1} \int_{\mathbb{R}}\left(\epsilon_{s}^{n}\right)^{-1}\left[\left(\rho^{n}\right)^{\prime}(z)-\frac{1+\alpha}{z} \rho^{n}(z)\right] \mu^{(n)}(\mathrm{d} s, \mathrm{~d} z)}{\epsilon_{1}^{n} \int_{0}^{1} \int_{\mathbb{R}}\left(\epsilon_{s}^{n}\right)^{-2} \rho^{n}(z) \mu^{(n)}(\mathrm{d} s, \mathrm{~d} z)}\right],
\end{aligned}
$$

and the remainder terms satisfy for any compact subset $Q \subset \mathbb{R} \times(0, \infty)$

$$
\forall p \geq 2, \quad \mathbb{E} \sup _{\beta \in Q}\left|\mathcal{R}_{1, \beta}^{n}(1)\right|^{p} \leq \frac{C}{n}, \quad \sup _{\beta \in Q}\left|\mathcal{R}_{2, \beta}^{n}(1)\right| \leq \frac{C}{n}, \quad \sup _{\beta \in Q}\left|\mathcal{R}_{3, \beta}^{n}(1)\right| \leq \frac{C}{n},
$$

where $C$ is some deterministic constant. 
Let us heuristically explain how this decomposition permits to establish the result of Theorem 2.2. Let $\left(\beta_{n}\right)$ be a sequence converging to $\beta$. First, from Lemma $4.2, n^{1 / \alpha}\left(\bar{Y}_{1}^{n, \beta_{n}, x_{0}}-\varsigma_{1}^{n, \theta_{n}, x_{0}}\right)$ converges almost surely to $\sigma L_{1}^{\alpha}$, this permits to check that $1_{\left\{n^{1 / \alpha}\left(\bar{Y}_{1}^{n, \beta_{n}, x_{0}}-\varsigma_{1}^{n, \theta_{n}, x_{0}}\right) \geq \sigma_{n} v\right\}}$ converges to $1_{\left\{L_{1}^{\alpha} \geq v\right\}}$. From Theorem 3.2, we deduce that $\frac{\sigma_{n}}{n^{1 / \alpha}} p_{\frac{1}{n}}^{\beta_{n}}\left(x_{0}, \frac{\sigma_{n}}{n^{1 / \alpha}} u+\varsigma_{1}^{n, \theta_{n}, x_{0}}\right)$ is close to $\mathbb{E} 1_{\left\{L_{1}^{\alpha} \geq u\right\}}\left[\widehat{\mathcal{H}}_{1, \beta_{n}}^{n}(1)+\widehat{\mathcal{H}}_{2, \beta_{n}}^{n}(1)\right]$ and it remains to study the limit of the main terms.

We can see from the definition of $\rho^{n}$ that $\rho^{n}(z) \stackrel{n \rightarrow \infty}{\longrightarrow} \rho(z)$ where

$$
\rho(z)= \begin{cases}z^{4} & \text { if }|z|<1 \\ \zeta(z) & \text { if } 1 \leq|z| \leq 2 \\ z^{2} & \text { if }|z|>2\end{cases}
$$

Combining this with Lemma 3.1, it permits to establish the almost sure convergence of the main terms:

$$
\begin{aligned}
& \widehat{\mathcal{H}}_{1, \beta_{n}}^{n}(1) \stackrel{n \rightarrow \infty}{\underset{\text { a.s. }}{\longrightarrow}} \mathcal{H}_{1, L^{\alpha}}(1), \\
& \widehat{\mathcal{H}}_{2, \beta_{n}}^{n}(1) \stackrel{n \rightarrow \infty}{\text { a.s. }} \mathcal{H}_{2, L^{\alpha}}(1),
\end{aligned}
$$

where $\mathcal{H}_{1, L^{\alpha}}(1), \mathcal{H}_{2, L^{\alpha}}(1)$ are given by

$$
\begin{aligned}
\mathcal{H}_{1, L^{\alpha}}(1) & =\frac{\int_{0}^{1} \int_{\mathbb{R}} \rho(z) \rho^{\prime}(z) \mu(\mathrm{d} s, \mathrm{~d} z)}{\left[\int_{0}^{1} \int_{\mathbb{R}} \rho(z) \mu(\mathrm{d} s, \mathrm{~d} z)\right]^{2}}, \\
\mathcal{H}_{2, L^{\alpha}}(1) & =-\frac{\int_{0}^{1} \int_{\mathbb{R}}\left[\rho^{\prime}(z)-\frac{1+\alpha}{z} \rho(z)\right] \mu(\mathrm{d} s, \mathrm{~d} z)}{\int_{0}^{1} \int_{\mathbb{R}} \rho(z) \mu(\mathrm{d} s, \mathrm{~d} z)} .
\end{aligned}
$$

Moreover, the limit weight $\mathcal{H}_{1, L^{\alpha}}(1)+\mathcal{H}_{2, L^{\alpha}}(1)$ can be interpreted as a Malliavin weight (see (4.30)) and we have the following representation for the density of $L_{1}^{\alpha}$

$$
\varphi_{\alpha}(u)=\mathbb{E} 1_{[u, \infty)}\left(L_{1}^{\alpha}\right)\left[\mathcal{H}_{1, L^{\alpha}}(1)+\mathcal{H}_{2, L^{\alpha}}(1)\right]
$$

This suggests that, as $n$ goes to infinity, $\frac{\sigma_{n}}{n^{1 / \alpha}} p_{\frac{1}{n}}^{\beta_{n}}\left(x_{0}, \frac{\sigma_{n}}{n^{1 / \alpha}} u+\varsigma_{1}^{n, \theta_{n}, x_{0}}\right)$ is close to $\varphi_{\alpha}$.

This is rigorously established in the next section.

\section{Proof of Theorems 3.2 And 2.2}

\subsection{Proof of Theorem 3.2}

The proof is based on the Malliavin calculus developed in Section A. We recall that $q^{n, \beta, x_{0}}$ is the density of $\bar{Y}_{1}^{n, \beta, x_{0}}$ and that the connection between the densities of $X_{\frac{1}{n}}^{\beta}$ and $\bar{Y}_{1}^{n, \beta, x_{0}}$ is given by $p_{1 / n}^{\beta}\left(x_{0}, x\right)=q^{n, \beta, x_{0}}(x)$.

We use the framework of Sections A.1 and A.2, with $g(z):=F_{n}(z)=\frac{1}{|z|^{1+\alpha}} \tau\left(\frac{z}{n^{1 / \alpha}}\right)$ and with the auxiliary function $\rho^{n}$ defined by (3.11) such that it satisfies all conditions of Section A.1. From the assumptions on $\tau$, we can easily deduce that $z^{2} \tau\left(\frac{z}{2 n^{1 / \alpha}}\right)=\left\{\begin{array}{lll}z^{2} & \text { if } & 2 \leq|z| \leq 2 n^{1 / \alpha} \\ 0 & \text { if } & |z|>4 n^{1 / \alpha}\end{array}\right.$

Moreover, we recall that $\rho^{n}(z) \stackrel{n \rightarrow \infty}{\longrightarrow} \rho(z)$ where $\rho$ is defined by (3.17). Note that from the definitions of $\rho^{n}$ and $\rho$, we can easily see that $\rho^{n}(z)=\rho(z)$ if $|z| \leq 2 n^{1 / \alpha}$. 
Remark 4.1. The choice of the auxiliary function $\rho^{n}$ for $|z|<1$ ensures that the non-degeneracy condition (A.6) is satisfied. It will appear later that the choice of the auxiliary function $\rho^{n}$ for $|z|>2$ permits to obtain Malliavin weights sufficiently integrable to compensate the lack of integrability of $L_{1}^{\alpha}$ (see Rem. 5.6 below).

The equation (3.8) (defining $\left.\left(\bar{Y}_{t}^{n, \beta, x_{0}}\right)\right)$ is a particular case of (A.1) with the function $a$ and the constant $c$ given explicitly as

$$
a(x, \theta)=\frac{1}{n} b(x, \theta), \quad c=\frac{1}{n^{1 / \alpha}} .
$$

Under the assumptions $\mathbf{H}_{1}$, we can apply the results of Theorem A.2 to $\bar{Y}_{1}^{n, \beta, x_{0}}$. The non-degeneracy assumption is verified by the choice of $\rho^{n}(z)$ near zero (see (3.11)). Let us denote by $U_{t}^{n, \beta}=\Gamma\left[\bar{Y}_{t}^{n, \beta, x_{0}}, \bar{Y}_{t}^{n, \beta, x_{0}}\right]$ and $W_{t}^{n, \beta}=$ $\Gamma\left[\bar{Y}_{t}^{n, \beta, x_{0}}, U_{t}^{n, \beta}\right]$, then we obtain:

$$
p_{\frac{1}{n}}^{\beta}\left(x_{0}, u\right)=q^{n, \beta, x_{0}}(u)=\mathbb{E}\left(1_{\left\{\bar{Y}_{1}^{n, \beta, x_{0}} \geq u\right\}} \mathcal{H}_{\bar{Y}_{1}^{n, \beta, x_{0}}}(1)\right)
$$

with

$$
\mathcal{H}_{\bar{Y}_{1}^{n, \beta, x_{0}}}(1)=\frac{W_{1}^{n, \beta}}{\left(U_{1}^{n, \beta}\right)^{2}}-2 \frac{L \bar{Y}_{1}^{n, \beta, x_{0}}}{U_{1}^{n, \beta}}
$$

Applying the results of Theorem A.2 and solving the linear equations (A.8)-(A.10) (with $a$ and $c$ given by (4.1)) we get,

$$
\begin{gathered}
U_{1}^{n, \beta}=\frac{\left(\epsilon_{1}^{n}\right)^{2} \sigma^{2}}{n^{2 / \alpha}} \int_{0}^{1} \int_{\mathbb{R}}\left(\epsilon_{s}^{n}\right)^{-2} \rho^{n}(z) \mu^{(n)}(\mathrm{d} s, \mathrm{~d} z) \\
L\left(\bar{Y}_{1}^{n, \beta, x_{0}}\right)= \\
\frac{\epsilon_{1}^{n}}{2 n} \int_{0}^{1} b^{\prime \prime}\left(\bar{Y}_{s}^{n, \beta, x_{0}}, \theta\right)\left(U_{s}^{n, \beta}\right)\left(\epsilon_{s}^{n}\right)^{-1} \mathrm{~d} s \\
+\frac{\sigma \epsilon_{1}^{n}}{2 n^{1 / \alpha}} \int_{0}^{1} \int_{\mathbb{R}}\left(\epsilon_{s}^{n}\right)^{-1}\left[\left(\rho^{n}\right)^{\prime}(z)+\frac{F_{n}^{\prime}(z)}{F_{n}(z)} \rho^{n}(z)\right] \mu^{(n)}(\mathrm{d} s, \mathrm{~d} z), \\
W_{1}^{n, \beta}=\frac{\sigma^{3}\left(\epsilon_{1}^{n}\right)^{3}}{n^{3 / \alpha}} \int_{0}^{1} \int_{\mathbb{R}}\left(\epsilon_{s}^{n}\right)^{-3}\left(\rho^{n}\right)^{\prime}(z) \rho^{n}(z) \mu^{(n)}(\mathrm{d} s, \mathrm{~d} z)+\frac{2\left(\epsilon_{1}^{n}\right)^{3}}{n} \int_{0}^{1} b^{\prime \prime}\left(\bar{Y}_{s}^{n, \beta, x_{0}}, \theta\right)\left(U_{s}^{n, \beta}\right)^{2}\left(\epsilon_{s}^{n}\right)^{-3} \mathrm{~d} s .
\end{gathered}
$$

Recalling that $F_{n}(z)=\frac{1}{|z|^{1+\alpha}} \tau\left(\frac{z}{n^{1 / \alpha}}\right)$ (see (3.1)), then $\frac{F_{n}^{\prime}(z)}{F_{n}(z)}=-\frac{1+\alpha}{z}+\frac{\tau^{\prime}\left(z / n^{1 / \alpha}\right)}{\tau\left(z / n^{1 / \alpha}\right)} \frac{1}{n^{1 / \alpha}}$ if $|z| \leq 2 n^{1 / \alpha}$. Based on these expressions and (4.2) we deduce, after some calculus, the decomposition (3.13), where the remainder terms are given by,

$$
\begin{aligned}
& \mathcal{R}_{1, \beta}^{n}(1)=-\frac{\int_{0}^{1} \int_{\mathbb{R}}\left(\epsilon_{s}^{n}\right)^{-1} \rho^{n}(z) \frac{\tau^{\prime}\left(z / n^{1 / \alpha}\right)}{\tau\left(z / n^{1 / \alpha}\right)} \mu^{(n)}(\mathrm{d} s, \mathrm{~d} z)}{\sigma \epsilon_{1}^{n} \int_{0}^{1} \int_{\mathbb{R}}\left(\epsilon_{s}^{n}\right)^{-2} \rho^{n}(z) \mu^{(n)}(\mathrm{d} s, \mathrm{~d} z)}, \\
& \mathcal{R}_{2, \beta}^{n}(1)=\frac{2\left(\epsilon_{1}^{n}\right)^{3} \int_{0}^{1} b^{\prime \prime}\left(\bar{Y}_{s}^{n, \beta, x_{0}}, \theta\right)\left(U_{s}^{n, \beta}\right)^{2}\left(\epsilon_{s}^{n}\right)^{-3} \mathrm{~d} s}{n\left(U_{1}^{n, \beta}\right)^{2}},
\end{aligned}
$$




$$
\mathcal{R}_{3, \beta}^{n}(1)=-\frac{\left(\epsilon_{1}^{n}\right) \int_{0}^{1} b^{\prime \prime}\left(\bar{Y}_{s}^{n, \beta, x_{0}}, \theta\right)\left(U_{s}^{n, \beta}\right)\left(\epsilon_{s}^{n}\right)^{-1} \mathrm{~d} s}{2 n\left(U_{1}^{n, \beta}\right)} .
$$

We can establish the following bounds for the remainder terms.

For $\mathcal{R}_{1, \beta}^{n}(1)$, since $\left(\epsilon_{s}^{n}\right)$ is lower and upper bounded uniformly with respect to $\beta$ (recall (3.10)), and since $\tau^{\prime}(z)=0$ on $[-1,1]$ then for $M$ a positive constant we have

$$
\sup _{\beta \in Q}\left|\mathcal{R}_{1, \beta}^{n}(1)\right| \leq M\left(\frac{\int_{0}^{1} \int_{|z|>2} z^{2}\left|\frac{\tau^{\prime}\left(z / n^{1 / \alpha}\right)}{\tau\left(z / n^{1 / \alpha}\right)}\right| \mu^{(n)}(\mathrm{d} s, \mathrm{~d} z)}{\int_{0}^{1} \int_{|z|>2} z^{2} \mu^{(n)}(\mathrm{d} s, \mathrm{~d} z)}\right) .
$$

Assume that there exists a jump of the Lévy process $L_{1}^{n}$ in $\left[-2 n^{1 / \alpha},-n^{1 / \alpha}\right) \cup\left(n^{1 / \alpha}, 2 n^{1 / \alpha}\right]$, then we get $\int_{0}^{1} \int_{|z|>2} z^{2} \mu^{(n)}(\mathrm{d} s, \mathrm{~d} z)>n^{2 / \alpha}$. Thus,

$$
\frac{\int_{0}^{1} \int_{|z|>2} z^{2}\left|\frac{\tau^{\prime}\left(z / n^{1 / \alpha}\right)}{\tau\left(z / n^{1 / \alpha}\right)}\right| \mu^{(n)}(\mathrm{d} s, \mathrm{~d} z)}{\int_{0}^{1} \int_{|z|>2} z^{2} \mu^{(n)}(\mathrm{d} s, \mathrm{~d} z)} \leq \int_{0}^{1} \int_{|z|>2}\left(\frac{z}{n^{1 / \alpha}}\right)^{2}\left|\frac{\tau^{\prime}\left(z / n^{1 / \alpha}\right)}{\tau\left(z / n^{1 / \alpha}\right)}\right| \mu^{(n)}(\mathrm{d} s, \mathrm{~d} z) .
$$

Assume that there are no jumps in $\left[-2 n^{1 / \alpha},-n^{1 / \alpha}\right) \cup\left(n^{1 / \alpha}, 2 n^{1 / \alpha}\right]$, since $\tau\left(z / n^{1 / \alpha}\right)=1$ if $|z| \leq n^{1 / \alpha}$, then $\tau^{\prime}\left(z / n^{1 / \alpha}\right)=0$ and as a consequence, the right-hand side of (4.9) equals zero in this case.

In both cases, for any $p \geq 1$

$$
\mathbb{E}\left(\frac{\int_{0}^{1} \int_{|z|>2} z^{2}\left|\frac{\tau^{\prime}\left(z / n^{1 / \alpha}\right)}{\tau\left(z / n^{1 / \alpha}\right)}\right| \mu^{(n)}(\mathrm{d} s, \mathrm{~d} z)}{\int_{0}^{1} \int_{|z|>2} z^{2} \mu^{(n)}(\mathrm{d} s, \mathrm{~d} z)}\right)^{p} \leq \mathbb{E}\left(\int_{0}^{1} \int_{|z|>2}\left(\frac{z}{n^{1 / \alpha}}\right)^{2}\left|\frac{\tau^{\prime}\left(z / n^{1 / \alpha}\right)}{\tau\left(z / n^{1 / \alpha}\right)}\right| \mu^{(n)}(\mathrm{d} s, \mathrm{~d} z)\right)^{p}
$$

Now from $\mu^{(n)}(\mathrm{d} s, \mathrm{~d} z)=\tilde{\mu}^{(n)}(\mathrm{d} s, \mathrm{~d} z)+v^{(n)}(\mathrm{d} s, \mathrm{~d} z)$, by convexity inequality, we have for $C(p)$ a positive constant

$$
\begin{aligned}
\mathbb{E}\left[\int_{0}^{1} \int_{|z|>2}\left(\frac{z}{n^{1 / \alpha}}\right)^{2}\left|\frac{\tau^{\prime}\left(z / n^{1 / \alpha}\right)}{\tau\left(z / n^{1 / \alpha}\right)}\right| \mu^{(n)}(\mathrm{d} s, \mathrm{~d} z)\right]^{p} \leq & C(p) \mathbb{E}\left[\int_{0}^{1} \int_{|z|>2}\left(\frac{z}{n^{1 / \alpha}}\right)^{2}\left|\frac{\tau^{\prime}\left(z / n^{1 / \alpha}\right)}{\tau\left(z / n^{1 / \alpha}\right)}\right| \tilde{\mu}^{(n)}(\mathrm{d} s, \mathrm{~d} z)\right]^{p} \\
& +C(p)\left[\int_{0}^{1} \int_{|z|>2}\left(\frac{z}{n^{1 / \alpha}}\right)^{2}\left|\frac{\tau^{\prime}\left(z / n^{1 / \alpha}\right)}{\tau\left(z / n^{1 / \alpha}\right)}\right| v^{(n)}(\mathrm{d} s, \mathrm{~d} z)\right]^{p}
\end{aligned}
$$

Using Kunita's first inequality (see Thm. 4.4.23 in [1]) for $p \geq 2$, there exists a constant $D(p)>0$ such that

$$
\begin{aligned}
\mathbb{E}\left[\int_{0}^{1} \int_{|z|>2}\left(\frac{z}{n^{1 / \alpha}}\right)^{2}\left|\frac{\tau^{\prime}\left(z / n^{1 / \alpha}\right)}{\tau\left(z / n^{1 / \alpha}\right)}\right| \tilde{\mu}^{(n)}(\mathrm{d} s, \mathrm{~d} z)\right]^{p} \leq & D(p)\left[\int_{0}^{1} \int_{|z|>2}\left(\frac{z}{n^{1 / \alpha}}\right)^{4}\left|\frac{\tau^{\prime}\left(z / n^{1 / \alpha}\right)}{\tau\left(z / n^{1 / \alpha}\right)}\right|^{2} v^{(n)}(\mathrm{d} s, \mathrm{~d} z)\right]^{p / 2} \\
& +D(p)\left[\int_{0}^{1} \int_{|z|>2}\left(\frac{z}{n^{1 / \alpha}}\right)^{2 p}\left|\frac{\tau^{\prime}\left(z / n^{1 / \alpha}\right)}{\tau\left(z / n^{1 / \alpha}\right)}\right|^{p} v^{(n)}(\mathrm{d} s, \mathrm{~d} z)\right] \\
= & \frac{D(p)}{n^{p / 2}}\left[\int_{0}^{1} \int_{1}^{2}\left(\frac{1}{u^{\alpha-3}}\left|\frac{\tau^{\prime}(u)}{\tau(u)}\right|^{2} \tau(u)\right) \mathrm{d} u \mathrm{~d} s\right]^{p / 2} \\
& +\left[\frac{D(p)}{n} \int_{0}^{1} \int_{1}^{2}\left(\frac{1}{u^{\alpha+1-2 p}}\left|\frac{\tau^{\prime}(u)}{\tau(u)}\right|^{p} \tau(u)\right) \mathrm{d} u \mathrm{~d} s\right]
\end{aligned}
$$


where we have used that $v^{(n)}(\mathrm{d} s, \mathrm{~d} z)=\mathrm{d} s \frac{1}{|z|^{1+\alpha}} \tau\left(z / n^{1 / \alpha}\right) \mathrm{d} z$ and the change of variable $u=\frac{z}{n^{1 / \alpha}}$.

Moreover, we have

$$
\begin{aligned}
{\left[\int_{0}^{1} \int_{|z|>2}\left(\frac{z}{n^{1 / \alpha}}\right)^{2}\left|\frac{\tau^{\prime}\left(z / n^{1 / \alpha}\right)}{\tau\left(z / n^{1 / \alpha}\right)}\right| v^{(n)}(\mathrm{d} s, \mathrm{~d} z)\right]^{p} } & =\left[\int_{0}^{1} \int_{|z|>2}\left(\frac{z}{n^{1 / \alpha}}\right)^{2}\left|\frac{\tau^{\prime}\left(z / n^{1 / \alpha}\right)}{\tau\left(z / n^{1 / \alpha}\right)}\right| \frac{1}{|z|^{1+\alpha}} \tau\left(z / n^{1 / \alpha}\right) \mathrm{d} z \mathrm{~d} s\right]^{p} \\
& =\left[\frac{1}{n} \int_{0}^{1} \int_{1}^{2} \frac{1}{u^{\alpha-1}}\left|\frac{\tau^{\prime}(u)}{\tau(u)}\right| \tau(u) \mathrm{d} u \mathrm{~d} s\right]^{p}
\end{aligned}
$$

Under the assumption $\mathbf{H}_{1}\left(b_{i i}\right)$, we deduce $\mathbb{E} \sup _{\beta \in Q}\left|\mathcal{R}_{1, \beta}^{n}(1)\right|^{p} \leq C / n, \forall p \geq 2$.

Using that $b$ has bounded derivatives and that $\sup _{\beta \in Q} \sup _{0 \leq s \leq 1} \frac{U_{s}^{n, \beta}}{U_{1}^{n, \beta}}$ is bounded, the remainder terms $R_{2, \beta}^{n}(1), R_{3, \beta}^{n}(1)$ satisfy the upper bound

$$
\sup _{\beta \in Q}\left|R_{2, \beta}^{n}(1)\right| \leq \frac{C}{n}, \quad \sup _{\beta \in Q}\left|R_{3, \beta}^{n}(1)\right| \leq \frac{C}{n}
$$

where $C$ is some deterministic constant.

\subsection{Proof of Theorem 2.2}

We first prove that $n^{1 / \alpha}\left(\bar{Y}_{1}^{n, \beta, x_{0}}-\varsigma_{1}^{n, \theta, x_{0}}\right)$ is close to a stable Lévy process.

Lemma 4.2. Let $\left(\varsigma_{t}^{n, \theta, x_{0}}\right)$ be the solution to the ordinary differential equation (2.1), then

$$
n^{1 / \alpha}\left(\bar{Y}_{1}^{n, \beta_{n}, x_{0}}-\varsigma_{1}^{n, \theta_{n}, x_{0}}\right) \stackrel{n \rightarrow \infty}{\text { a.s. }} \sigma L_{1}^{\alpha},
$$

for any sequence $\left(\beta_{n}\right)$ converging to $\beta$, and this convergence is uniform with respect to $x_{0}$.

Proof. Using (3.8) and the boundedness of $b^{\prime}$, we have for $t \in[0,1]$

$$
\begin{aligned}
\left|n^{1 / \alpha}\left(\bar{Y}_{t}^{n, \beta_{n}, x_{0}}-\varsigma_{t}^{n, \theta_{n}, x_{0}}\right)-\sigma L_{t}^{\alpha}\right|= & \left|\frac{1}{n} \int_{0}^{t} n^{1 / \alpha}\left[b\left(\bar{Y}_{s}^{n, \beta_{n}, x_{0}}, \theta_{n}\right)-b\left(\varsigma_{s}^{n, \theta_{n}, x_{0}}, \theta_{n}\right)\right] \mathrm{d} s+\left[\sigma_{n} L_{t}^{n}-\sigma L_{t}^{\alpha}\right]\right| \\
\leq & \frac{1}{n} \int_{0}^{t}\left\|b^{\prime}\right\|_{\infty}\left[\left|n^{1 / \alpha}\left(\bar{Y}_{s}^{n, \beta_{n}, x_{0}}-\varsigma_{s}^{n, \theta_{n}, x_{0}}\right)-\sigma L_{s}^{\alpha}\right|\right] \mathrm{d} s \\
& +\frac{\sigma\left\|b^{\prime}\right\|_{\infty}}{n} \int_{0}^{1}\left|L_{s}^{\alpha}\right| \mathrm{d} s+\sup _{t \in[0,1]}\left|\sigma_{n} L_{t}^{n}-\sigma L_{t}^{\alpha}\right|,
\end{aligned}
$$

where $\left\|b^{\prime}\right\|_{\infty}=\sup _{x, \theta}\left|b^{\prime}(x, \theta)\right|$. Applying Gronwall's Lemma, we get

$$
\sup _{x_{0}}\left|n^{1 / \alpha}\left(\bar{Y}_{1}^{n, \beta_{n}, x_{0}}-\varsigma_{1}^{n, \theta_{n}, x_{0}}\right)-\sigma L_{1}^{\alpha}\right| \leq C\left[\frac{\sigma}{n} \int_{0}^{1}\left|L_{s}^{\alpha}\right| \mathrm{d} s+\sup _{t \in[0,1]}\left|\sigma_{n} L_{t}^{n}-\sigma L_{t}^{\alpha}\right|\right]
$$

where $\mathrm{C}$ is a positive constant.

From Lemma 3.1, we have $\sup _{t \in[0,1]}\left|L_{t}^{n}-L_{t}^{\alpha}\right| \stackrel{\text { a.s. }}{\longrightarrow} 0$ and we deduce $\sup _{t \in[0,1]}\left|\sigma_{n} L_{t}^{n}-\sigma L_{t}^{\alpha}\right| \stackrel{\text { a.s. }}{\longrightarrow} 0$. Since $t \mapsto L_{t}^{\alpha}$ is càdlàg, we get $\int_{0}^{1}\left|L_{s}^{\alpha}\right| \mathrm{d} s<\infty$ a.s., then $\frac{\sigma}{n} \int_{0}^{1}\left|L_{s}^{\alpha}\right| \mathrm{d} s \stackrel{\text { a.s. }}{\longrightarrow} 0$ and we get the result of Lemma 4.2. 
We now proceed to the proof of Theorem 2.2. From (3.9) and Theorem 3.2, we have the representation

$\frac{\sigma_{n}}{n^{1 / \alpha}} p_{\frac{1}{n}}^{\beta_{n}}\left(x_{0}, \frac{u \sigma_{n}}{n^{1 / \alpha}}+\varsigma_{1}^{n, \theta_{n}, x_{0}}\right)=\frac{\sigma_{n}}{n^{1 / \alpha}} q^{n, \beta, x_{0}}\left(\frac{u \sigma_{n}}{n^{1 / \alpha}}+\varsigma_{1}^{n, \theta_{n}, x_{0}}\right)=\mathbb{E}\left(1_{\left\{\bar{Y}_{1}^{n, \beta_{n}, x_{0}} \geq \frac{u \sigma_{n}}{n^{1 / \alpha}}+\varsigma_{1}^{n, \theta_{n}, x_{0}}\right\}} \frac{\sigma_{n}}{n^{1 / \alpha}} \mathcal{H}_{\bar{Y}_{1}^{n, \beta_{n}, x_{0}}}(1)\right)$,

where $\frac{\sigma_{n}}{n^{1 / \alpha}} \mathcal{H}_{\bar{Y}_{1}^{n, \beta_{n}, x_{0}}}(1)=\widehat{\mathcal{H}}_{1, \beta_{n}}^{n}(1)+\widehat{\mathcal{H}}_{2, \beta_{n}}^{n}(1)+\frac{\sigma_{n}}{n^{1 / \alpha}} \mathcal{R}_{1, \beta_{n}}^{n}(1)+\frac{\sigma_{n}}{n^{1 / \alpha}} \mathcal{R}_{2, \beta_{n}}^{n}(1)+\frac{\sigma_{n}}{n^{1 / \alpha}} \mathcal{R}_{3, \beta_{n}}^{n}(1)$, with $\widehat{\mathcal{H}}_{1, \beta_{n}}^{n}(1)$, $\widehat{\mathcal{H}}_{2, \beta_{n}}^{n}(1)$ given by (3.14), (3.15) and $\mathcal{R}_{1, \beta_{n}}^{n}(1), \mathcal{R}_{2, \beta_{n}}^{n}(1), \mathcal{R}_{3, \beta_{n}}^{n}(1)$ satisfy the bounds (3.16).

Since $b^{\prime}$ is bounded and $\rho^{n}(z) \geq 0$, we deduce the upper bounds

$$
\begin{aligned}
& \left|\widehat{\mathcal{H}}_{1, \beta_{n}}^{n}(1)\right| \leq C^{*}\left[\frac{\int_{0}^{1} \int_{\mathbb{R}} \rho^{n}(z)\left|\left(\rho^{n}\right)^{\prime}(z)\right| \mu^{(n)}(\mathrm{d} s, \mathrm{~d} z)}{\left[\int_{0}^{1} \int_{\mathbb{R}} \rho^{n}(z) \mu^{(n)}(\mathrm{d} s, \mathrm{~d} z)\right]^{2}}\right], \\
& \left|\widehat{\mathcal{H}}_{2, \beta_{n}}^{n}(1)\right| \leq C^{*}\left[\frac{\int_{0}^{1} \int_{\mathbb{R}}\left[\left|\rho^{n^{\prime}}(z)\right|+\frac{1+\alpha}{|z|} \rho^{n}(z)\right] \mu^{(n)}(\mathrm{d} s, \mathrm{~d} z)}{\int_{0}^{1} \int_{\mathbb{R}} \rho^{n}(z) \mu^{(n)}(\mathrm{d} s, \mathrm{~d} z)}\right],
\end{aligned}
$$

for some constant $C^{*}>0$.

We now show that $\sup _{n}\left|\widehat{\mathcal{H}}_{1, \beta_{n}}^{n}(1)\right|^{p}$ and $\sup _{n}\left|\widehat{\mathcal{H}}_{2, \beta_{n}}^{n}(1)\right|^{p}$ are integrable $\forall p \geq 1$. The proof will be divided into the two following steps.

Step 1: We show that the right-hand side of (4.17) is bounded by a random variable independent of $n$ and belonging to $\cap_{p \geq 1} \mathbf{L}^{p}$. In fact, since the measures $\mu^{(n)}$ and $\mu$ coincide on the set $\left\{(s, z)|s \in[0,1]| z \mid, \leq n^{1 / \alpha}\right\}$, and $\rho^{n}(z)=\rho(z)$ on the support of the Poisson measure $\mu^{(n)}$, we have

$$
\frac{\int_{0}^{1} \int_{\mathbb{R}} \rho^{n}(z)\left|\left(\rho^{n}\right)^{\prime}(z)\right| \mu^{(n)}(\mathrm{d} s, \mathrm{~d} z)}{\left[\int_{0}^{1} \int_{\mathbb{R}} \rho^{n}(z) \mu^{(n)}(\mathrm{d} s, \mathrm{~d} z)\right]^{2}} \leq\left(\frac{\int_{0}^{1} \int_{|z| \leq 2}\left|\rho^{\prime}(z)\right| \rho(z) \mu(\mathrm{d} s, \mathrm{~d} z)}{\left(\int_{0}^{1} \int_{|z| \leq 2} \rho(z) \mu(\mathrm{d} s, \mathrm{~d} z)\right)^{2}}+\frac{\int_{0}^{1} \int_{|z|>2} 2|z|^{3} \mu^{(n)}(\mathrm{d} s, \mathrm{~d} z)}{\left(\int_{0}^{1} \int_{|z|>2} z^{2} \mu^{(n)}(\mathrm{d} s, \mathrm{~d} z)\right)^{2}}\right) .
$$

We first consider the first term in the right-hand side of (4.19). Since $\rho, \rho^{\prime} \in \cap_{p \geq 1} \mathbf{L}^{p}\left(1_{|z| \leq 2}|z|^{-1-\alpha} \mathrm{d} z\right)$, we get

$$
\mathbb{E}\left[\left(\int_{0}^{1} \int_{|z| \leq 2}\left|\rho^{\prime}(z)\right| \rho(z) \mu(\mathrm{d} s, \mathrm{~d} z)\right)^{p}\right]<\infty, \quad \forall p \geq 1
$$

On the other hand, since $\rho$ satisfies the non degeneracy assumption (A.6), $\left[\int_{0}^{1} \int_{|z| \leq 2} \rho(z) \mu(\mathrm{d} s, \mathrm{~d} z)\right]^{-1}$ belongs to $\cap_{p \geq 1} \mathbf{L}^{p}$ (see [5], Thm. 4, p.2323), we deduce that the first term of (4.19) belongs to $\cap_{p \geq 1} \mathbf{L}^{p}$, moreover, it does not depend on $n$.

Turning to the second term in the right-hand side of (4.19), since $v^{(n)}(\{(t, z)|0 \leq t \leq 1| z \mid,>2\})<\infty$, we have the following representation (see Chap. VI in [4])

$$
\begin{aligned}
\int_{0}^{1} \int_{|z|>2} 2|z|^{3} \mu^{(n)}(\mathrm{d} s, \mathrm{~d} z) & =\sum_{i=1}^{N_{1}} 2\left|Z_{i}\right|^{3} \quad \text { a.s. } \\
\int_{0}^{1} \int_{|z|>2} z^{2} \mu^{(n)}(\mathrm{d} s, \mathrm{~d} z) & =\sum_{i=1}^{N_{1}} Z_{i}^{2} \quad \text { a.s. }
\end{aligned}
$$


where $N=\left(N_{t}\right)_{1 \geq t \geq 0}$ is a Poisson process with intensity $\lambda_{n}=\int_{|z|>2} F_{n}(z) \mathrm{d} z<\infty$, and $\left(Z_{i}\right)_{i \geq 0}$ are i.i.d. random variables independent of $N$ with probability measure $\frac{F_{n}(z) 1_{|z|>2} \mathrm{~d} z}{\lambda_{n}}$. Thus,

$$
\frac{\int_{0}^{1} \int_{|z|>2} 2|z|^{3} \mu^{(n)}(\mathrm{d} s, \mathrm{~d} z)}{\left(\int_{0}^{1} \int_{|z|>2} z^{2} \mu^{(n)}(\mathrm{d} s, \mathrm{~d} z)\right)^{2}}=\frac{\sum_{i=1}^{N_{1}} 2\left|Z_{i}\right|^{3}}{\left(\sum_{i=1}^{N_{1}} Z_{i}^{2}\right)^{2}} \leq \frac{\sum_{i=1}^{N_{1}} 2\left|Z_{i}\right|^{3}}{\sum_{i=1}^{N_{1}} Z_{i}^{4}} \leq 1
$$

where we used $Z_{i}^{2} \geq 0$ and $\left|Z_{i}\right|>2$. We deduce that $\sup _{n}\left|\widehat{\mathcal{H}}_{1, \beta_{n}}^{n}(1)\right|^{p}$ is integrable $\forall p \geq 1$.

Step 2: We show that $\sup _{n}\left|\widehat{\mathcal{H}}_{2, \beta_{n}}^{n}(1)\right|^{p}$ is integrable.

Using the definitions of $\rho^{n}$ (recall (3.11)), $\rho$ (recall (3.17)) and $\rho^{n}=\rho$ on the support of the Poisson measure $\mu^{(n)}$ (see Sect. 3.2), we have

$$
\begin{aligned}
\frac{\int_{0}^{1} \int_{\mathbb{R}}\left[\left|\rho^{n^{\prime}}(z)\right|+\frac{1+\alpha}{|z|} \rho^{n}(z)\right] \mu^{(n)}(\mathrm{d} s, \mathrm{~d} z)}{\int_{0}^{1} \int_{\mathbb{R}} \rho^{n}(z) \mu^{(n)}(\mathrm{d} s, \mathrm{~d} z)} \leq & \frac{\int_{0}^{1} \int_{|z| \leq 2}\left(\left|\rho(z)^{\prime}\right|+\rho(z) \frac{1+\alpha}{|z|}\right) \mu(\mathrm{d} s, \mathrm{~d} z)}{\int_{0}^{1} \int_{|z| \leq 2} \rho(z) \mu(\mathrm{d} s, \mathrm{~d} z)} \\
& +\frac{\int_{0}^{1} \int_{|z|>2}(3+\alpha)|z| \mu^{(n)}(\mathrm{d} s, \mathrm{~d} z)}{\int_{0}^{1} \int_{|z|>2} z^{2} \mu^{(n)}(\mathrm{d} s, \mathrm{~d} z)}
\end{aligned}
$$

where we used $\int_{0}^{1} \int_{|z|<2} \rho(z) \mu(\mathrm{d} s, \mathrm{~d} z) \geq 0, \int_{0}^{1} \int_{|z|>2} \rho^{n}(z) \mu^{(n)}(\mathrm{d} s, \mathrm{~d} z) \geq 0$, and the equality of $\mu^{(n)}$ and $\mu$ on the set $\left\{(s, z)|s \in[0,1]| z \mid, \leq n^{1 / \alpha}\right\}$.

Proceeding as for the first term in the right-hand side of (4.19), we also get that the first term of (4.22) belongs to $\cap_{p \geq 1} \mathbf{L}^{p}$.

On the other hand, for the second term of (4.22) we have:

$$
\frac{\int_{0}^{1} \int_{|z|>2}(3+\alpha)|z| \mu^{(n)}(\mathrm{d} s, \mathrm{~d} z)}{\int_{0}^{1} \int_{|z|>2} z^{2} \mu^{(n)}(\mathrm{d} s, \mathrm{~d} z)} \leq \frac{\int_{0}^{1} \int_{|z|>2}(3+\alpha) z^{2} \mu^{(n)}(\mathrm{d} s, \mathrm{~d} z)}{\int_{0}^{1} \int_{|z|>2} z^{2} \mu^{(n)}(\mathrm{d} s, \mathrm{~d} z)}=3+\alpha .
$$

This completes the proof of Step 2 .

We finally deduce (with additionally some uniformity with respect to $x_{0}$ )

$$
\forall p \geq 1, \quad \mathbb{E}\left(\sup _{n, \beta, x_{0}}\left|\widehat{\mathcal{H}}_{1, \beta}^{n}(1)\right|^{p}+\sup _{n, \beta, x_{0}}\left|\widehat{\mathcal{H}}_{2, \beta}^{n}(1)\right|^{p}\right)<\infty
$$

Recalling the almost sure convergences (3.18) and (3.19), we get from the dominated convergence theorem the $\mathbf{L}^{p}$-convergences

$$
\begin{aligned}
& \widehat{\mathcal{H}}_{1, \beta_{n}}^{n}(1) \stackrel{n \rightarrow \infty}{\mathbf{L}^{p}} \mathcal{H}_{1, L^{\alpha}}(1), \quad \forall p \geq 1 . \\
& \widehat{\mathcal{H}}_{2, \beta_{n}}^{n}(1) \stackrel{n \rightarrow \infty}{\mathbf{L}^{p}} \mathcal{H}_{2, L^{\alpha}}(1), \quad \forall p \geq 1,
\end{aligned}
$$

where $\mathcal{H}_{1, L^{\alpha}}(1)$ and $\mathcal{H}_{2, L^{\alpha}}(1)$ are defined respectively by (3.20) and (3.21). 
On the other hand, Lemma 4.2 implies that $n^{1 / \alpha}\left(\bar{Y}_{1}^{n, \beta_{n}, x_{0}}-\varsigma_{1}^{n, \theta_{n}, x_{0}}\right)$ converges almost surely to $\sigma L_{1}^{\alpha}$. Then, using $P\left(L_{1}^{\alpha}=u\right)=0$, we deduce the almost sure convergence

$$
1_{\left\{\bar{Y}_{1}^{n, \beta_{n}, x_{0}} \geq \frac{u \sigma_{n}}{n^{1 / \alpha}}+\varsigma_{1}^{n, \theta_{n}, x_{0}}\right\}}=1_{[u, \infty)}\left(\frac{n^{1 / \alpha}\left(\bar{Y}_{1}^{n, \beta_{n}, x_{0}}-\varsigma_{1}^{n, \theta_{n}, x_{0}}\right)}{\sigma_{n}}\right) \underset{\text { a.s. }}{\stackrel{n \rightarrow \infty}{\longrightarrow}} 1_{[u, \infty)}\left(L_{1}^{\alpha}\right) .
$$

Applying the dominated convergence theorem, we get the latter convergence in $\mathbf{L}^{p}, \forall p \geq 1$. This gives finally :

$$
\frac{\sigma_{n}}{n^{1 / \alpha}} q^{n, \beta_{n}, x_{0}}\left(\frac{u \sigma_{n}}{n^{1 / \alpha}}+\varsigma_{1}^{n, \theta_{n}, x_{0}}\right) \stackrel{n \rightarrow \infty}{\longrightarrow} \mathbb{E}\left[1_{[u, \infty)}\left(L_{1}^{\alpha}\right) \mathcal{H}_{L^{\alpha}}(1)\right]
$$

where $\mathcal{H}_{L^{\alpha}}(1)=\mathcal{H}_{1, L^{\alpha}}(1)+\mathcal{H}_{2, L^{\alpha}}(1)$ and $\mathcal{H}_{1, L^{\alpha}}(1), \mathcal{H}_{2, L^{\alpha}}(1)$ are given by (3.20), (3.21), respectively. Remark that, we also get from (3.12), (3.13), (3.16) and (4.25), (4.26)

$$
\sup _{u \in \mathbb{R}} \sup _{n} \frac{\sigma_{n}}{n^{1 / \alpha}} q^{n, \beta_{n}, x_{0}}\left(\frac{u \sigma_{n}}{n^{1 / \alpha}}+\varsigma_{1}^{n, \theta_{n}, x_{0}}\right)<\infty .
$$

To finish the proof of the convergence, it remains to show that

$$
\varphi_{\alpha}(u)=\mathbb{E}\left[1_{[u, \infty)}\left(L_{1}^{\alpha}\right) \mathcal{H}_{L^{\alpha}}(1)\right] .
$$

Let us denote by $\varphi^{n}(u)$ the density of the variable $L_{1}^{n}$. We consider the situation where the drift function $b \equiv 0$ and $x_{0}=0$ for which $n^{1 / \alpha} \bar{Y}_{1}^{n, \beta, x_{0}}=\sigma L_{1}^{n}$. Then (4.28), (4.29) yield

$$
\begin{gathered}
\varphi^{n}(u) \stackrel{n \rightarrow \infty}{\longrightarrow} \mathbb{E}\left[1_{[u, \infty)}\left(L_{1}^{\alpha}\right) \mathcal{H}_{L^{\alpha}}(1)\right]:=\psi(u), \\
\sup _{u \in \mathbb{R}} \sup _{n} \varphi^{n}(u)<\infty .
\end{gathered}
$$

Assume by contradiction that, for some $u$, we have $\psi(u) \neq \varphi_{\alpha}(u)$. Since $P\left(L_{1}^{\alpha}=u\right)=0$, it can be seen that $\psi$ is continuous at the point $u$. Hence, one can find a continuous, compactly supported, function $f$ such that

$$
\int f(x) \psi(x) d x \neq \int f(x) \varphi_{\alpha}(x) d x .
$$

On the one hand we have, $\mathbb{E}\left[f\left(L_{1}^{n}\right)\right]=\int f(x) \varphi^{n}(x) d x \stackrel{n \rightarrow \infty}{\longrightarrow} \int f(x) \psi(x) d x$ where we have used the dominated convergence theorem with (4.31)-(4.32). On the other hand, we can write

$$
\mathbb{E}\left[f\left(L_{1}^{n}\right)\right]=\mathbb{E}\left[f\left(L_{1}^{n}\right) 1_{\left\{L_{1}^{n}=L_{1}^{\alpha}\right\}}\right]+\mathbb{E}\left[f\left(L_{1}^{n}\right) 1_{\left\{L_{1}^{n} \neq L_{1}^{\alpha}\right\}}\right]
$$

By Lemma 3.1, we have $\mathbb{P}\left(L_{1}^{n}=L_{1}^{\alpha}\right) \stackrel{n \rightarrow \infty}{\longrightarrow} 1$. We deduce that,

$$
\mathbb{E}\left[f\left(L_{1}^{n}\right)\right] \stackrel{n \rightarrow \infty}{\longrightarrow} \mathbb{E}\left[f\left(L_{1}^{\alpha}\right)\right]=\int f(x) \varphi_{\alpha}(x) d x .
$$

This last convergence result clearly contradicts (4.33) and we get (4.30).

Combining the preceding results with (4.28), we can get the results of Theorem 2.2. 


\section{Proof of Theorem 2.5}

The proof is divided into three steps. We first give a representation of the derivative of the density and explicit the iterated Malliavin weights based on the calculus given in Section A. We then study the convergence of these weights and proceed to the proof of Theorem 2.5.

\subsection{Representation of $\nabla_{\beta} p_{\frac{1}{n}}^{\beta_{n}}$ and computation of the iterated Malliavin weights}

We intensively use the results of Section A. From Theorem A.4, we have the representation

$$
\begin{aligned}
\nabla_{\beta} p_{\frac{1}{n}}^{\beta_{n}}\left(x_{0}, \frac{u \sigma_{n}}{n^{1 / \alpha}}+\varsigma_{1}^{n, \theta_{n}, x_{0}}\right) & =\nabla_{\beta} q^{n, \beta_{n}, x_{0}}\left(\frac{u \sigma_{n}}{n^{1 / \alpha}}+\varsigma_{1}^{n, \theta_{n}, x_{0}}\right) \\
& =\mathbb{E}\left[1_{\left\{\bar{Y}_{1}^{n, \beta_{n}, x_{0}} \geq \frac{u \sigma_{n}}{n^{1 / \alpha}}+\varsigma_{1}^{n, \theta_{n}, x_{0}}\right\}} \mathcal{H}_{\bar{Y}_{1}^{n, \beta_{n}, x_{0}}}\left(\mathcal{H}_{\bar{Y}_{1}^{n, \beta_{n}, x_{0}}}\left(\nabla_{\beta} \bar{Y}_{1}^{n, \beta_{n}, x_{0}}\right)\right)\right] .
\end{aligned}
$$

Using (A.7), (A.14) and (A.18), (A.19), by some simple calculus, we get the explicit formula for the iterated Malliavin weight

$$
\begin{aligned}
& \mathcal{H}_{\bar{Y}_{1}^{n, \beta, x_{0}}}\left(\mathcal{H}_{\bar{Y}_{1}^{n, \beta, x_{0}}}\left(\nabla_{\beta} \bar{Y}_{1}^{n, \beta, x_{0}}\right)\right)=\left(\begin{array}{c}
\partial_{\theta} \bar{Y}_{1}^{n, \beta, x_{0}} \\
\partial_{\sigma} \bar{Y}_{1}^{n, \beta, x_{0}}
\end{array}\right) \mathcal{H}_{\bar{Y}_{1}^{n, \beta, x_{0}}}(1)^{2}-\left(\begin{array}{c}
V_{1}^{n, \theta} \\
V_{1}^{n, \sigma}
\end{array}\right) \frac{2 \mathcal{H}_{\bar{Y}_{1}^{n, \beta, x_{0}}(1)}}{U_{1}^{n, \beta}}+\left(\begin{array}{l}
\partial_{\theta} \bar{Y}_{1}^{n, \beta, x_{0}} \\
\partial_{\sigma} \bar{Y}_{1}^{n, \beta, x_{0}}
\end{array}\right)
\end{aligned}
$$

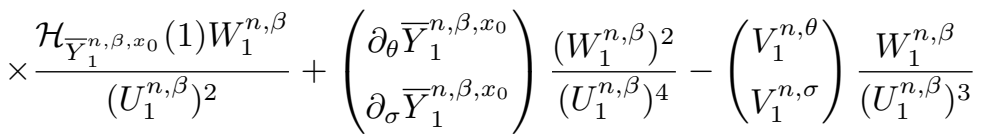

$$
\begin{aligned}
& +\left(\begin{array}{c}
\partial_{\theta} \bar{Y}_{1}^{n, \beta, x_{0}} \\
\partial_{\sigma} \bar{Y}_{1}^{n, \beta, x_{0}}
\end{array}\right) \frac{2 D_{1}^{n, \beta}}{\left(U_{1}^{n, \beta}\right)^{2}}-\left(\begin{array}{c}
\partial_{\theta} \bar{Y}_{1}^{n, \beta, x_{0}} \\
\partial_{\sigma} \bar{Y}_{1}^{n, \beta, x_{0}}
\end{array}\right) \frac{Q_{1}^{n, \beta}}{\left(U_{1}^{n, \beta}\right)^{3}}+\left(\begin{array}{c}
T_{1}^{n, \theta} \\
T_{1}^{n, \sigma}
\end{array}\right) \frac{1}{\left(U_{1}^{n, \beta}\right)^{2}},
\end{aligned}
$$

where $\mathcal{H}_{\bar{Y}_{1}^{n, \beta, x_{0}}}^{n}(1), U_{1}^{n, \beta}, W_{1}^{n, \beta}$ are given by (3.13), (4.3), (4.5), respectively.

Moreover the processes $\left(\partial_{\theta} \bar{Y}_{t}^{n, \beta, x_{0}}\right)_{t}$ and $\left(\partial_{\sigma} \bar{Y}_{t}^{n, \beta, x_{0}}\right)_{t}$ are respectively solution to

$$
\begin{aligned}
\partial_{\theta} \bar{Y}_{t}^{n, \beta, x_{0}} & =\frac{1}{n} \int_{0}^{t} b^{\prime}\left(\bar{Y}_{s}^{n, \beta, x_{0}}, \theta\right) \partial_{\theta} \bar{Y}_{s}^{n, \beta, x_{0}} \mathrm{~d} s+\frac{1}{n} \int_{0}^{t} \partial_{\theta} b\left(\bar{Y}_{s}^{n, \beta, x_{0}}, \theta\right) \mathrm{d} s, \\
\partial_{\sigma} \bar{Y}_{t}^{n, \beta, x_{0}} & =\frac{1}{n} \int_{0}^{t} b^{\prime}\left(\bar{Y}_{s}^{n, \beta, x_{0}}, \theta\right) \partial_{\sigma} \bar{Y}_{s}^{n, \beta, x_{0}} \mathrm{~d} s+\frac{L_{t}^{n}}{n^{1 / \alpha}} .
\end{aligned}
$$

For the computations of $V_{1}^{n, \theta}=\Gamma\left(\bar{Y}_{1}^{n, \beta, x_{0}}, \partial_{\theta} \bar{Y}_{1}^{n, \beta, x_{0}}\right)$ and $V_{1}^{n, \sigma}=\Gamma\left(\bar{Y}_{1}^{n, \beta, x_{0}}, \partial_{\sigma} \bar{Y}_{1}^{n, \beta, x_{0}}\right)$, using (A.15), (A.16) we have

$$
\begin{aligned}
V_{1}^{n, \theta} & =\frac{1}{n}\left(\epsilon_{1}^{n}\right)^{2} \int_{0}^{1}\left(\epsilon_{s}^{n}\right)^{-2}\left(U_{s}^{n, \beta}\left[\left(\partial_{\theta} b\right)^{\prime}\left(\bar{Y}_{s}^{n, \beta, x_{0}}, \theta\right)+b^{\prime \prime}\left(\bar{Y}_{s}^{n, \beta, x_{0}}, \theta\right) \partial_{\theta} \bar{Y}_{s}^{n, \beta, x_{0}}\right]\right) \mathrm{d} s, \\
V_{1}^{n, \sigma} & =\frac{1}{n}\left(\epsilon_{1}^{n}\right)^{2} \int_{0}^{1}\left(\epsilon_{s}^{n}\right)^{-2}\left(b^{\prime \prime}\left(\bar{Y}_{s}^{n, \beta, x_{0}}, \theta\right) \partial_{\sigma} \bar{Y}_{s}^{n, \beta, x_{0}} U_{s}^{n, \beta}\right) \mathrm{d} s+\frac{\sigma}{n^{2 / \alpha}}\left(\epsilon_{1}^{n}\right)^{2} \int_{0}^{t} \int_{\mathbb{R}}\left(\epsilon_{s}^{n}\right)^{-2} \rho^{n}(z) \mu^{(n)}(\mathrm{d} s, \mathrm{~d} z) .
\end{aligned}
$$


Finally from ((A.20)-(A.23)) we compute explicitly $D_{1}^{n, \beta}=\Gamma\left(\bar{Y}_{1}^{n, \beta, x_{0}}, L \bar{Y}_{1}^{n, \beta, x_{0}}\right), Q_{1}^{n, \beta}=\Gamma\left(\bar{Y}_{1}^{n, \beta, x_{0}}, W_{1}^{n, \beta}\right)$, $T_{1}^{n, \theta}=\Gamma\left(\bar{Y}_{1}^{n, \beta, x_{0}}, V_{1}^{n, \theta}\right)$ and $T_{1}^{n, \sigma}=\Gamma\left(\bar{Y}_{1}^{n, \beta, x_{0}}, V_{1}^{n, \sigma}\right)$. We get:

$$
\begin{aligned}
& D_{1}^{n, \beta}=\frac{\left(\epsilon_{1}^{n}\right)^{2}}{n} \int_{0}^{1}\left(\epsilon_{s}^{n}\right)^{-2} b^{\prime \prime}\left(\bar{Y}_{s}^{n, \beta, x_{0}}, \theta\right) L \bar{Y}_{s}^{n, \beta, x_{0}} U_{s}^{n, \beta} \mathrm{d} s+\frac{\left(\epsilon_{1}^{n}\right)^{2}}{2 n} \int_{0}^{1}\left(\epsilon_{s}^{n}\right)^{-2} b^{\prime \prime}\left(\bar{Y}_{s}^{n, \beta, x_{0}}, \theta\right) W_{s}^{n, \beta} \mathrm{d} s \\
& +\frac{\left(\epsilon_{1}^{n}\right)^{2}}{2 n} \int_{0}^{1}\left(\epsilon_{s}^{n}\right)^{-2} b^{\prime \prime \prime}\left(\bar{Y}_{s}^{n, \beta, x_{0}}, \theta\right)\left(U_{s}^{n, \beta}\right)^{2} \mathrm{~d} s \\
& +\frac{\sigma^{2}\left(\epsilon_{1}^{n}\right)^{2}}{2 n^{2 / \alpha}} \int_{0}^{1} \int_{\mathbb{R}}\left(\epsilon_{s}^{n}\right)^{-2} \rho^{n}(z)\left(\left(\rho^{n}\right)^{\prime}(z)+\rho^{n}(z) \frac{F_{n}^{\prime}(z)}{F_{n}(z)}\right)^{\prime} \mu^{(n)}(\mathrm{d} s, \mathrm{~d} z), \\
& Q_{1}^{n, \beta}=\frac{7\left(\epsilon_{1}^{n}\right)^{4}}{n} \int_{0}^{1}\left(\epsilon_{1}^{n}\right)^{-4} b^{\prime \prime}\left(\bar{Y}_{s}^{n, \beta, x_{0}}, \theta\right) W_{s}^{n, \beta} U_{s}^{n, \beta} \mathrm{d} s+\frac{2\left(\epsilon_{1}^{n}\right)^{4}}{n} \int_{0}^{1}\left(\epsilon_{1}^{n}\right)^{-4} b^{\prime \prime \prime}\left(\bar{Y}_{s}^{n, \beta, x_{0}}, \theta\right)\left(U_{s}^{n, \beta}\right)^{3} \mathrm{~d} s \\
& +\frac{\sigma^{4}\left(\epsilon_{1}^{n}\right)^{4}}{n^{4 / \alpha}} \int_{0}^{1} \int_{\mathbb{R}}\left(\epsilon_{1}^{n}\right)^{-4} \rho^{n}(z)\left[\left(\left(\rho^{n}\right)^{\prime}(z)\right)^{2}+\rho^{n}(z)\left(\rho^{n}\right)^{\prime \prime}(z)\right] \mu^{(n)}(\mathrm{d} s, \mathrm{~d} z), \\
& T_{1}^{n, \theta}=\frac{3\left(\epsilon_{1}^{n}\right)^{3}}{n} \int_{0}^{1}\left(\epsilon_{1}^{n}\right)^{-3} b^{\prime \prime}\left(\bar{Y}_{s}^{n, \beta, x_{0}}, \theta\right) V_{s}^{n, \theta} U_{s}^{n, \beta} \mathrm{d} s+\frac{\left(\epsilon_{1}^{n}\right)^{3}}{n} \int_{0}^{1}\left(\epsilon_{1}^{n}\right)^{-3}\left(\partial_{\theta} b\right)^{\prime}\left(\bar{Y}_{s}^{n, \beta, x_{0}}, \theta\right) W_{s}^{n, \beta} \mathrm{d} s \\
& +\frac{\left(\epsilon_{1}^{n}\right)^{3}}{n} \int_{0}^{1}\left(\epsilon_{1}^{n}\right)^{-3} b^{\prime \prime}\left(\bar{Y}_{s}^{n, \beta, x_{0}}, \theta\right) \partial_{\theta} \bar{Y}_{s}^{n, \beta, x_{0}} W_{s}^{n, \beta} \mathrm{d} s+\frac{\left(\epsilon_{1}^{n}\right)^{3}}{n} \int_{0}^{1}\left(\epsilon_{1}^{n}\right)^{-3}\left(\partial_{\theta} b\right)^{\prime \prime}\left(\bar{Y}_{s}^{n, \beta, x_{0}}, \theta\right)\left(U_{s}^{n, \beta}\right)^{2} \mathrm{~d} s \\
& +\frac{\left(\epsilon_{1}^{n}\right)^{3}}{n} \int_{0}^{1}\left(\epsilon_{1}^{n}\right)^{-3} b^{\prime \prime \prime}\left(\bar{Y}_{s}^{n, \beta, x_{0}}, \theta\right) \partial_{\theta} \bar{Y}_{s}^{n, \beta, x_{0}}\left(U_{s}^{n, \beta}\right)^{2} \mathrm{~d} s, \\
& T_{1}^{n, \sigma}=\frac{3\left(\epsilon_{1}^{n}\right)^{3}}{n} \int_{0}^{1}\left(\epsilon_{1}^{n}\right)^{-3} b^{\prime \prime}\left(\bar{Y}_{s}^{n, \beta, x_{0}}, \theta\right) V_{s}^{n, \sigma} U_{s}^{n, \beta} \mathrm{d} s+\frac{\left(\epsilon_{1}^{n}\right)^{3}}{n} \int_{0}^{1}\left(\epsilon_{1}^{n}\right)^{-3} b^{\prime \prime}\left(\bar{Y}_{s}^{n, \beta, x_{0}}, \theta\right) \partial_{\sigma} \bar{Y}_{s}^{n, \beta, x_{0}} W_{s}^{n, \beta} \mathrm{d} s+\frac{\left(\epsilon_{1}^{n}\right)^{3}}{n} \\
& \times \int_{0}^{1}\left(\epsilon_{1}^{n}\right)^{-3} b^{\prime \prime \prime}\left(\bar{Y}_{s}^{n, \beta, x_{0}}, \theta\right) \partial_{\sigma} \bar{Y}_{s}^{n, \beta, x_{0}}\left(U_{s}^{n, \beta}\right)^{2} \mathrm{~d} s+\frac{\sigma^{2}\left(\epsilon_{1}^{n}\right)^{3}}{n^{3 / \alpha}} \int_{0}^{1} \int_{\mathbb{R}}\left(\epsilon_{1}^{n}\right)^{-3} \rho^{n}(z)\left(\rho^{n}\right)^{\prime}(z) \mu^{(n)}(\mathrm{d} s, \mathrm{~d} z) .
\end{aligned}
$$

From the above calculus and combining with (5.1) and (5.2) we have an explicit representation for the derivative of the density with respect to parameter $\beta$ that allows to analyze its asymptotic behavior in small time. To obtain the results of Theorem 2.5, we will study the convergence of each term appearing in the decompostion (5.2). This is based on the preceding explicit expressions that permit to identify some main terms and some remainder terms.

In the sequel, we prove that all the terms involving the derivatives of $b$ with respect to $x$ are remainder terms.

\subsection{Convergence of the iterated weights}

In this section, we study the convergence of the iterated Malliavin weight $\mathcal{H}_{\bar{Y}_{1}^{n, \beta_{n}, x_{0}}}\left(\mathcal{H}_{\bar{Y}_{1}^{n, \beta_{n}, x_{0}}}\left(\nabla_{\beta} \bar{Y}_{1}^{n, \beta_{n}, x_{0}}\right)\right)$ which is the cornerstone of the proof for the convergence of $\nabla_{\beta} p_{\frac{1}{n}}^{\beta_{n}}$ later. Firstly, we state some technical lemmas useful for our aim. The proofs of these lemmas are postponed to the end of the section.

We recall that $\left(\partial_{\theta} \bar{Y}_{t}^{n, \beta, x_{0}}\right)_{t}$ and $\left(\partial_{\sigma} \bar{Y}_{t}^{n, \beta, x_{0}}\right)_{t}$ are respectively solution to (5.3) and (5.4).

Lemma 5.1. We have for all compact subset $Q \subset \mathbb{R} \times(0, \infty)$ 
i) $\sup _{\beta \in Q}\left|\partial_{\theta} \bar{Y}_{1}^{n, \beta, x_{0}}\right| \leq \frac{C}{n}$, where $C$ is some deterministic constant.

ii) $\sup _{\beta \in Q} \sup _{s \in[0,1]}\left|\partial_{\sigma} \bar{Y}_{s}^{n, \beta, x_{0}}\right| \underset{L^{p}}{\stackrel{n \rightarrow \infty}{\longrightarrow}} 0, \quad \forall p \geq 1$.

Lemma 5.2. Let $\left(\beta_{n}\right)$ be a sequence converging to $\beta$ and $Q$ be a compact subset of $\mathbb{R} \times(0, \infty)$, the following decompositions and estimates hold,
i) $\frac{1}{n^{1 / \alpha}} \frac{D_{1}^{n, \beta}}{\left(U_{1}^{n, \beta}\right)^{2}}=\frac{n^{1 / \alpha}}{2 \sigma^{2}} \widehat{\mathcal{H}}_{3, \beta}^{n}(1)+\mathcal{R}_{4, \beta}^{n}(1)+\mathcal{R}_{5, \beta}^{n}(1)+\mathcal{R}_{6, \beta}^{n}(1)$.
ii) $\frac{1}{n^{1 / \alpha}} \frac{Q_{1}^{n, \beta}}{\left(U_{1}^{n, \beta}\right)^{3}}=\frac{n^{1 / \alpha}}{\sigma^{2}} \widehat{\mathcal{H}}_{4, \beta}^{n}(1)+\mathcal{R}_{7, \beta}^{n}(1)+\mathcal{R}_{8, \beta}^{n}(1)$.
iii) $\sup _{\beta \in Q} \frac{1}{n^{1 / \alpha+1}} \frac{\sup _{s \in[0,1]}\left|\partial_{\sigma} \bar{Y}_{s}^{n, \beta, x_{0}} W_{s}^{n, \beta}\right|}{\left(U_{1}^{n, \beta}\right)^{2}} \frac{n \rightarrow \infty}{L^{p}} 0, \quad \forall p \geq 1$.

The main terms $\widehat{\mathcal{H}}_{3, \beta}^{n}(1), \widehat{\mathcal{H}}_{4, \beta}^{n}(1)$ are given by

$$
\begin{aligned}
& \widehat{\mathcal{H}}_{3, \beta}^{n}(1)=\frac{\int_{0}^{1} \int_{\mathbb{R}}\left(\epsilon_{s}^{n}\right)^{-2} \rho^{n}(z)\left[\left(\rho^{n}\right)^{\prime \prime}(z)-\left(\rho^{n}\right)^{\prime}(z) \frac{(1+\alpha)}{z}+\rho^{n}(z) \frac{(1+\alpha)}{z^{2}}\right] \mu^{(n)}(\mathrm{d} s, \mathrm{~d} z)}{\left(\epsilon_{1}^{n}\right)^{2}\left(\int_{0}^{1} \int_{\mathbb{R}}\left(\epsilon_{s}^{n}\right)^{-2} \rho^{n}(z) \mu^{(n)}(\mathrm{d} s, \mathrm{~d} z)\right)^{2}}, \\
& \widehat{\mathcal{H}}_{4, \beta}^{n}(1)=\frac{\int_{0}^{1} \int_{\mathbb{R}}\left(\epsilon_{1}^{n}\right)^{-4} \rho^{n}(z)\left[\left(\left(\rho^{n}\right)^{\prime}(z)\right)^{2}+\rho^{n}(z)\left(\rho^{n}\right)^{\prime \prime}(z)\right] \mu^{(n)}(\mathrm{d} s, \mathrm{~d} z)}{\left(\epsilon_{1}^{n}\right)^{2}\left(\int_{0}^{1} \int_{\mathbb{R}}\left(\epsilon_{s}^{n}\right)^{-2} \rho^{n}(z) \mu^{(n)}(\mathrm{d} s, \mathrm{~d} z)\right)^{3}},
\end{aligned}
$$

where $\left(\epsilon_{s}^{n}\right)_{s \in[0,1]}$ is given by (3.10). Moreover we have for $p \geq 1$ and some deterministic constant $C$

$$
\begin{aligned}
& \widehat{\mathcal{H}}_{3, \beta_{n}}^{n}(1) \stackrel{n \rightarrow \infty}{L^{p}} \mathcal{H}_{3, L^{\alpha}}(1), \quad \widehat{\mathcal{H}}_{4, \beta_{n}}^{n}(1) \stackrel{n \rightarrow \infty}{L^{p}} \mathcal{H}_{4, L^{\alpha}}(1), \\
& \sup _{\beta \in Q}\left|\mathcal{R}_{4, \beta}^{n}(1)\right| \frac{n \rightarrow \infty}{L^{p}} 0, \quad \sup _{\beta \in Q}\left|\mathcal{R}_{5, \beta}^{n}(1)\right| \frac{n \rightarrow \infty}{L^{p}} 0, \quad \sup _{\beta \in Q}\left|\mathcal{R}_{6, \beta}^{n}(1)\right| \leq \frac{C}{2 n^{1+1 / \alpha}}, \\
& \sup _{\beta \in Q}\left|\mathcal{R}_{7, \beta}^{n}(1)\right| \frac{n \rightarrow \infty}{L^{p}} 0, \quad \sup _{\beta \in Q}\left|\mathcal{R}_{8, \beta}^{n}(1)\right| \leq \frac{C}{n^{1+1 / \alpha}},
\end{aligned}
$$

where

$$
\begin{aligned}
& \mathcal{H}_{3, L^{\alpha}}(1)=\frac{\int_{0}^{1} \int_{\mathbb{R}}\left(\rho(z) \rho^{\prime \prime}(z)-\rho(z) \rho^{\prime}(z) \frac{(1+\alpha)}{z}+(\rho(z))^{2} \frac{(1+\alpha)}{z^{2}}\right) \mu(\mathrm{d} s, \mathrm{~d} z)}{\left(\int_{0}^{1} \int_{\mathbb{R}} \rho(z) \mu(\mathrm{d} s, \mathrm{~d} z)\right)^{2}}, \\
& \mathcal{H}_{4, L^{\alpha}}(1)=\frac{\int_{0}^{1} \int_{\mathbb{R}} \rho(z)\left[\left(\rho^{\prime}(z)\right)^{2}+\rho(z) \rho^{\prime \prime}(z)\right] \mu(\mathrm{d} s, \mathrm{~d} z)}{\left(\int_{0}^{1} \int_{\mathbb{R}} \rho(z) \mu(\mathrm{d} s, \mathrm{~d} z)\right)^{3}} .
\end{aligned}
$$

Lemma 5.3. Let $\left(\beta_{n}\right)$ be a sequence converging to $\beta$ and $Q$ be a compact subset of $\mathbb{R} \times(0, \infty)$, the following estimates hold:

$$
\begin{aligned}
& \text { i) } \sup _{\beta \in Q}\left|\frac{V_{1}^{n, \theta}}{U_{1}^{n, \beta}}\right| \leq \frac{C}{n}, \\
& \text { ii) } \sup _{\beta \in Q} \frac{1}{n^{2 / \alpha-1}} \frac{\left|T_{1}^{n, \theta}\right|}{\left(U_{1}^{n, \beta}\right)^{2}} \frac{n \rightarrow \infty}{L^{p}} 0, \quad \forall p \geq 1,
\end{aligned}
$$


iii) $\frac{V_{1}^{n, \sigma}}{U_{1}^{n, \beta}}=\frac{1}{\sigma}+\mathcal{R}_{9, \beta}^{n}(1)$,

iv) $\frac{1}{n^{1 / \alpha}} \frac{T_{1}^{n, \sigma}}{\left(U_{1}^{n, \beta}\right)^{2}}=\frac{1}{\sigma^{2}} \widehat{\mathcal{H}}_{5, \beta}^{n}(1)+\mathcal{R}_{10, \beta}^{n}(1)+\mathcal{R}_{11, \beta}^{n}(1)+\mathcal{R}_{12, \beta}^{n}(1)$,

where $C$ is some deterministic constant. The main term $\widehat{\mathcal{H}}_{5, \beta}^{n}(1)$ is given by

$$
\widehat{\mathcal{H}}_{5, \beta}^{n}(1)=\frac{\int_{0}^{1} \int_{\mathbb{R}}\left(\epsilon_{1}^{n}\right)^{-3} \rho^{n}(z)\left(\rho^{n}\right)^{\prime}(z) \mu^{(n)}(\mathrm{d} s, \mathrm{~d} z)}{\epsilon_{1}^{n}\left(\int_{0}^{1} \int_{\mathbb{R}}\left(\epsilon_{s}^{n}\right)^{-2} \rho^{n}(z) \mu^{(n)}(\mathrm{d} s, \mathrm{~d} z)\right)^{2}}
$$

with $\left(\epsilon_{s}^{n}\right)$ given by (3.10). Moreover, $\left(\sup _{\beta \in Q}\left|\mathcal{R}_{i, \beta}^{n}(1)\right|\right)_{9 \leq i \leq 12}$ converge to zero as $n \rightarrow \infty$ in $\boldsymbol{L}^{p}, \forall p \geq 1$ and $\widehat{\mathcal{H}}_{5, \beta_{n}}^{n}(1) \underset{L^{p}, \forall p \geq 1}{\stackrel{n \rightarrow \infty}{\longrightarrow}} \mathcal{H}_{5, L^{\alpha}}(1)$, with

$$
\mathcal{H}_{5, L^{\alpha}}(1)=\frac{\int_{0}^{1} \int_{\mathbb{R}} \rho(z) \rho^{\prime}(z) \mu(\mathrm{d} s, \mathrm{~d} z)}{\left(\int_{0}^{1} \int_{\mathbb{R}} \rho(z) \mu(\mathrm{d} s, \mathrm{~d} z)\right)^{2}} .
$$

Lemma 5.4. Let $\left(\beta_{n}\right)$ be a sequence converging to $\beta$. For all $p \geq 1$, the following convergences hold uniformly with respect to $x_{0}$ :

$$
\begin{gathered}
n \partial_{\theta} \bar{Y}_{1}^{n, \beta_{n}, x_{0}}\left(\widehat{\mathcal{H}}_{\beta_{n}}^{n}(1)\right)^{q} \underset{L^{p}}{n \rightarrow \infty} \partial_{\theta} b\left(x_{0}, \theta\right)\left(\mathcal{H}_{L^{\alpha}}(1)\right)^{q}, \quad \forall q \geq 1, \\
n \partial_{\theta} \bar{Y}_{1}^{n, \beta_{n}, x_{0}} \widehat{\mathcal{H}}_{\beta_{n}}^{n}(1) \widehat{\mathcal{H}}_{1, \beta_{n}}^{n}(1) \frac{n \rightarrow \infty}{L^{p}} \partial_{\theta} b\left(x_{0}, \theta\right) \mathcal{H}_{L^{\alpha}}(1) \mathcal{H}_{1, L^{\alpha}}(1), \\
n^{1 / \alpha} \partial_{\sigma} \bar{Y}_{1}^{n, \beta_{n}, x_{0}}\left(\widehat{\mathcal{H}}_{\beta_{n}}^{n}(1)\right)^{q} \stackrel{n \rightarrow \infty}{L^{p}} L_{1}^{\alpha}\left(\mathcal{H}_{L^{\alpha}}(1)\right)^{q}, \quad \forall q \geq 1, \\
n^{1 / \alpha} \partial_{\sigma} \bar{Y}_{1}^{n, \beta_{n}, x_{0}} \widehat{\mathcal{H}}_{\beta_{n}}^{n}(1) \widehat{\mathcal{H}}_{1, \beta_{n}}^{n}(1) \frac{n \rightarrow \infty}{L^{p}} L_{1}^{\alpha} \mathcal{H}_{L^{\alpha}}(1) \mathcal{H}_{1, L^{\alpha}}(1),
\end{gathered}
$$

where $\widehat{\mathcal{H}}_{\beta}^{n}(1)=\widehat{\mathcal{H}}_{1, \beta}^{n}(1)+\widehat{\mathcal{H}}_{2, \beta}^{n}(1)$ with $\widehat{\mathcal{H}}_{1, \beta}^{n}(1), \widehat{\mathcal{H}}_{2, \beta}^{n}(1)$ given by $(3.14),(3.15) ; \mathcal{H}_{L^{\alpha}}(1)=\mathcal{H}_{1, L^{\alpha}}(1)+\mathcal{H}_{2, L^{\alpha}}(1)$ where $\mathcal{H}_{1, L^{\alpha}}(1), \mathcal{H}_{2, L^{\alpha}}(1)$ are defined by $(3.20)$, (3.21).

Lemma 5.5. Let $\left(\beta_{n}\right)$ be a sequence converging to $\beta$. For all $p \geq 1$ the following convergences hold uniformly with respect to $x_{0}$ :

$$
\begin{aligned}
& \text { i) } n \partial_{\theta} \bar{Y}_{1}^{n, \beta_{n}, x_{0}} \widehat{\mathcal{H}}_{3, \beta_{n}}^{n}(1) \stackrel{n \rightarrow \infty}{\stackrel{L^{p}}{\longrightarrow}} \partial_{\theta} b\left(x_{0}, \theta\right) \mathcal{H}_{3, L^{\alpha}}(1), \\
& \text { ii) } n \partial_{\theta} \bar{Y}_{1}^{n, \beta_{n}, x_{0}} \widehat{\mathcal{H}}_{4, \beta_{n}}^{n}(1) \stackrel{n \rightarrow \infty}{L^{p}} \partial_{\theta} b\left(x_{0}, \theta\right) \mathcal{H}_{4, L^{\alpha}}(1), \\
& \text { iii) } n^{1 / \alpha} \partial_{\sigma} \bar{Y}_{1}^{n, \beta_{n}, x_{0}} \widehat{\mathcal{H}}_{3, \beta_{n}}^{n}(1) \stackrel{n \rightarrow \infty}{\longrightarrow} L_{1}^{\alpha} \mathcal{H}_{3, L^{\alpha}}(1), \\
& \text { iv) } n^{1 / \alpha} \partial_{\sigma} \bar{Y}_{1}^{n, \beta_{n}, x_{0}} \widehat{\mathcal{H}}_{4, \beta_{n}}^{n}(1) \stackrel{n \rightarrow \infty}{L^{p}} L_{1}^{\alpha} \mathcal{H}_{4, L^{\alpha}}(1),
\end{aligned}
$$

where $\widehat{\mathcal{H}}_{3, \beta}^{n}(1), \widehat{\mathcal{H}}_{4, \beta}^{n}(1)$ are given by (5.11), (5.12), and $\mathcal{H}_{3, L^{\alpha}}(1), \mathcal{H}_{4, L^{\alpha}}(1)$, are defined by (5.16), (5.17).

The uniform convergence with respect to $x_{0}$ is not required in this paper but will be useful in [6]. 
Remark 5.6. We observe that although $L_{1}^{\alpha}$ does not belong to $\mathbf{L}^{p}$, the choice of the auxiliary function $\rho$ permits to prove that $L_{1}^{\alpha}\left(\mathcal{H}_{L^{\alpha}}(1)\right)^{2}, L_{1}^{\alpha} \mathcal{H}_{3, L^{\alpha}}(1)$ and $L_{1}^{\alpha} \mathcal{H}_{4, L^{\alpha}}(1)$ belong to $\mathbf{L}^{p}, \forall p \geq 1$.

Based on the preceding lemmas, we can prove the following convergence result.

Proposition 5.7. Let $\left(\beta_{n}\right)_{n \geq 1}$ be a sequence such that $\beta_{n} \stackrel{n \rightarrow \infty}{\longrightarrow} \beta$ then for all $p \geq 1$

$$
\begin{gathered}
\frac{\sigma_{n}^{2}}{n^{1 / \alpha}} \mathcal{H}_{\bar{Y}_{1}^{n, \beta_{n}, x_{0}}}\left(\mathcal{H}_{\bar{Y}_{1}^{n, \beta_{n}, x_{0}}}\left(\partial_{\sigma} \bar{Y}_{1}^{n, \beta_{n}, x_{0}}\right)\right) \underset{\boldsymbol{L}^{p}}{\longrightarrow} \mathcal{H}^{(2)} \\
\frac{\sigma_{n}^{2}}{n^{2 / \alpha-1}} \mathcal{H}_{\bar{Y}_{1}^{n, \beta_{n}, x_{0}}}\left(\mathcal{H}_{\bar{Y}_{1}^{n, \beta_{n}, x_{0}}}\left(\partial_{\theta} \bar{Y}_{1}^{n, \beta_{n}, x_{0}}\right)\right) \frac{n \rightarrow \infty}{\boldsymbol{L}^{p}} \partial_{\theta} b\left(x_{0}, \theta\right) \mathcal{H}_{1}^{(2)},
\end{gathered}
$$

where $\mathcal{H}^{(2)}$ and $\mathcal{H}_{1}^{(2)}$ are some random variables whose expressions do not depend on $\beta$ and $b$.

Proof. From the equation (5.2), we have

$$
\begin{aligned}
& \left(\begin{array}{c}
\frac{\sigma_{n}^{2}}{n^{2 / \alpha-1}} \mathcal{H}_{\bar{Y}_{1}^{n, \beta_{n}, x_{0}}}\left(\mathcal{H}_{\bar{Y}_{1}^{n, \beta_{n}, x_{0}}}\left(\partial_{\theta} \bar{Y}_{1}^{n, \beta_{n}, x_{0}}\right)\right) \\
\frac{\sigma_{n}^{2}}{n^{1 / \alpha}} \mathcal{H}_{\bar{Y}_{1}^{n, \beta_{n}, x_{0}}}\left(\mathcal{H}_{\bar{Y}_{1}^{n, \beta_{n}, x_{0}}}\left(\partial_{\sigma} \bar{Y}_{1}^{n, \beta_{n}, x_{0}}\right)\right)
\end{array}\right) \\
& =\left(\begin{array}{c}
\frac{\sigma_{n}^{2}}{n^{2 / \alpha-1}} \partial_{\theta} \bar{Y}_{1}^{n, \beta_{n}, x_{0}} \\
\frac{\sigma_{n}^{2}}{n^{1 / \alpha}} \partial_{\sigma} \bar{Y}_{1}^{n, \beta_{n}, x_{0}}
\end{array}\right) \mathcal{H}_{\bar{Y}_{1}^{n, \beta_{n}, x_{0}}}(1)^{2}-\left(\begin{array}{c}
\frac{\sigma_{n}^{2}}{n^{2 / \alpha-1}} V_{1}^{n, \theta_{n}} \\
\frac{\sigma_{n}^{2}}{n^{1 / \alpha}} V_{1}^{n, \sigma_{n}}
\end{array}\right) \frac{2 \mathcal{H}_{\bar{Y}_{1}^{n, \beta_{n}, x_{0}}(1)}}{\left(U_{1}^{n, \beta_{n}}\right)}+\left(\begin{array}{c}
\frac{\sigma_{n}^{2}}{n^{2 / \alpha-1}} \partial_{\theta} \bar{Y}_{1}^{n, \beta_{n}, x_{0}} \\
\frac{\sigma_{n}^{2}}{n^{1 / \alpha}} \partial_{\sigma} \bar{Y}_{1}^{n, \beta_{n}, x_{0}}
\end{array}\right)
\end{aligned}
$$

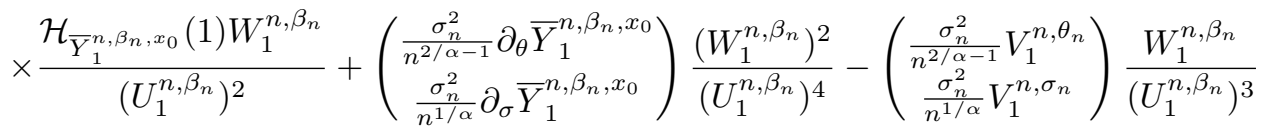

$$
\begin{aligned}
& +\left(\begin{array}{c}
\frac{\sigma_{n}^{2}}{n^{2 / \alpha-1}} \partial_{\theta} \bar{Y}_{1}^{n, \beta_{n}, x_{0}} \\
\frac{\sigma_{n}^{2}}{n^{1 / \alpha}} \partial_{\sigma} \bar{Y}_{1}^{n, \beta_{n}, x_{0}}
\end{array}\right) \frac{2 D_{1}^{n, \beta_{n}}}{\left(U_{1}^{n, \beta_{n}}\right)^{2}}-\left(\begin{array}{c}
\frac{\sigma_{n}^{2}}{n^{2 / \alpha-1}} \partial_{\theta} \bar{Y}_{1}^{n, \beta_{n}, x_{0}} \\
\frac{\sigma_{n}^{2}}{n^{1 / \alpha}} \partial_{\sigma} \bar{Y}_{1}^{n, \beta_{n}, x_{0}}
\end{array}\right) \frac{Q_{1}^{n, \beta_{n}}}{\left(U_{1}^{n, \beta_{n}}\right)^{3}}+\left(\begin{array}{c}
\frac{\sigma_{n}^{2}}{n^{2 / \alpha-1}} T_{1}^{n, \theta_{n}} \\
\frac{\sigma_{n}^{2}}{n^{1 / \alpha}} T_{1}^{n, \sigma_{n}}
\end{array}\right) \frac{1}{\left(U_{1}^{n, \beta_{n}}\right)^{2}} .
\end{aligned}
$$

We will prove the convergence of each term in the right-hand side of (5.26)

Term 1: Recall (3.13) and set $\widehat{\mathcal{H}}_{\beta_{n}}^{n}(1)=\widehat{\mathcal{H}}_{1, \beta_{n}}^{n}(1)+\widehat{\mathcal{H}}_{2, \beta_{n}}^{n}(1), \mathcal{R}_{\beta_{n}}^{n}(1)=\mathcal{R}_{2, \beta_{n}}^{n}(1)+\mathcal{R}_{3, \beta_{n}}^{n}(1)$. Remark that by (3.16), we have $\left|\mathcal{R}_{\beta_{n}}^{n}(1)\right| \leq \frac{C}{n}$ where $\mathrm{C}$ is some deterministic constant. Moreover, we can rewrite the first term as

$$
\begin{aligned}
& \left(\begin{array}{c}
\frac{\sigma_{n}^{2}}{n^{2 / \alpha-1}} \partial_{\theta} \bar{Y}_{1}^{n, \beta_{n}, x_{0}} \\
\frac{\sigma_{n}^{2}}{n^{1 / \alpha}} \partial_{\sigma} \bar{Y}_{1}^{n, \beta_{n}, x_{0}}
\end{array}\right)\left[\mathcal{H}_{\bar{Y}_{1}^{n, \beta_{n}, x_{0}}}(1)\right]^{2} \\
& =\left(\begin{array}{c}
\frac{\sigma_{n}^{2}}{n^{2 / \alpha-1}} \partial_{\theta} \bar{Y}_{1}^{n, \beta_{n}, x_{0}} \\
\frac{\sigma_{n}^{2}}{n^{1 / \alpha}} \partial_{\sigma} \bar{Y}_{1}^{n, \beta_{n}, x_{0}}
\end{array}\right)\left[\frac{n^{1 / \alpha}}{\sigma_{n}}\left[\widehat{\mathcal{H}}_{1, \beta_{n}}^{n}(1)+\widehat{\mathcal{H}}_{2, \beta_{n}}^{n}(1)\right]+\mathcal{R}_{1, \beta_{n}}^{n}(1)+\mathcal{R}_{2, \beta_{n}}^{n}(1)+\mathcal{R}_{3, \beta_{n}}^{n}(1)\right]^{2} \\
& =\left(\begin{array}{c}
n \partial_{\theta} \bar{Y}_{1}^{n, \beta_{n}, x_{0}} \\
n^{1 / \alpha} \partial_{\sigma} \bar{Y}_{1}^{n, \beta_{n}, x_{0}}
\end{array}\right) \widehat{\mathcal{H}}_{\beta_{n}}^{n}(1)^{2}+\left(\begin{array}{c}
\frac{2 \sigma_{n}}{n^{1 / \alpha-1}} \partial_{\theta} \bar{Y}_{1}^{n, \beta_{n}, x_{0}} \\
2 \sigma_{n} \partial_{\sigma} \bar{Y}_{1}^{n, \beta_{n}, x_{0}}
\end{array}\right) \widehat{\mathcal{H}}_{\beta_{n}}^{n}(1) \mathcal{R}_{\beta_{n}}^{n}(1)+\left(\begin{array}{c}
\frac{2 \sigma_{n}}{n^{1 / \alpha-1}} \partial_{\theta} \bar{Y}_{1}^{n, \beta_{n}, x_{0}} \\
2 \sigma_{n} \partial_{\sigma} \bar{Y}_{1}^{n, \beta_{n}, x_{0}}
\end{array}\right) \widehat{\mathcal{H}}_{\beta_{n}}^{n}(1) \\
& \times \mathcal{R}_{1, \beta_{n}}^{n}(1)+\left(\begin{array}{c}
\frac{\sigma_{n}^{2}}{n^{2 / \alpha-1}} \partial_{\theta} \bar{Y}_{1}^{n, \beta_{n}, x_{0}} \\
\frac{\sigma_{n}^{2}}{n^{1 / \alpha}} \partial_{\sigma} \bar{Y}_{1}^{n, \beta_{n}, x_{0}}
\end{array}\right) \mathcal{R}_{1, \beta_{n}}^{n}(1)^{2}+\left(\begin{array}{c}
\frac{\sigma_{n}^{2}}{n^{2 / \alpha-1}} \partial_{\theta} \bar{Y}_{1}^{n, \beta_{n}, x_{0}} \\
\frac{\sigma_{n}^{2}}{n^{1 / \alpha}} \partial_{\sigma} \bar{Y}_{1}^{n, \beta_{n}, x_{0}}
\end{array}\right)\left(\mathcal{R}_{\beta_{n}}^{n}(1)\right)^{2}+\left(\begin{array}{c}
\frac{2 \sigma_{n}^{2}}{n^{2 / \alpha-1}} \partial_{\theta} \bar{Y}_{1}^{n, \beta_{n}, x_{0}} \\
\frac{2 \sigma_{n}^{2}}{n^{1 / \alpha}} \partial_{\sigma} \bar{Y}_{1}^{n, \beta_{n}, x_{0}}
\end{array}\right) \\
& \times \mathcal{R}_{1, \beta_{n}}^{n}(1) \mathcal{R}_{\beta_{n}}^{n}(1) \text {, }
\end{aligned}
$$


where $\mathcal{R}_{1, \beta_{n}}^{n}(1)$ is given by (4.6). We can deduce from (3.16), (4.25), (4.26), Lemma 5.4 and Lemma 5.1 that

$$
\left(\begin{array}{c}
\frac{\sigma_{n}^{2}}{n^{2 / \alpha-1}} \partial_{\theta} \bar{Y}_{1}^{n, \beta_{n}, x_{0}} \\
\frac{\sigma_{n}^{2}}{n^{1 / \alpha}} \partial_{\sigma} \bar{Y}_{1}^{n, \beta_{n}, x_{0}}
\end{array}\right)\left(\mathcal{H}_{\bar{Y}_{1}^{n, \beta_{n}, x_{0}}}(1)\right)^{2} \underset{\mathbf{L}^{p}, \forall p \geq 1}{\stackrel{n \rightarrow \infty}{\longrightarrow}}\left(\begin{array}{c}
\partial_{\theta} b\left(x_{0}, \theta\right) \\
L_{1}^{\alpha}
\end{array}\right)\left(\mathcal{H}_{L^{\alpha}}(1)\right)^{2}
$$

Term 2: From (3.13) and Lemma 5.3 part $i$ ) and part iii), we can estimate the second term as

$$
\begin{aligned}
& \left(\begin{array}{c}
\frac{\sigma_{n}^{2}}{n^{2 / \alpha-1}} V_{1}^{n, \theta_{n}} \\
\frac{\sigma_{n}^{2}}{n^{1 / \alpha}} V_{1}^{n, \sigma_{n}}
\end{array}\right) \frac{2 \mathcal{H}_{\bar{Y}_{1}^{n, \beta_{n}, x_{0}}}^{n}(1)}{\left(U_{1}^{n, \beta_{n}}\right)}=\left(\begin{array}{c}
O\left(\frac{1}{n^{2 / \alpha}}\right) \\
\frac{2 \sigma_{n}^{2}}{n^{1 / \alpha}}\left[\mathcal{R}_{9, \beta_{n}}^{n}(1)+\frac{1}{\sigma_{n}}\right]
\end{array}\right)\left[\frac{1}{\frac{1}{\sigma_{n}}} n^{1 / \alpha} \widehat{\mathcal{H}}_{\beta_{n}}^{n}(1)+\mathcal{R}_{1, \beta_{n}}^{n}(1)+\mathcal{R}_{\beta_{n}}^{n}(1)\right] \\
& =\left(\begin{array}{c}
O\left(\frac{1}{n^{1 / \alpha}}\right) \widehat{\mathcal{H}}_{\beta_{n}}^{n}(1)+O\left(\frac{1}{n^{2 / \alpha}}\right) \mathcal{R}_{1, \beta_{n}}^{n}(1)+O\left(\frac{1}{n^{2 / \alpha}}\right) \mathcal{R}_{\beta_{n}}^{n}(1) \\
2 \widehat{\mathcal{H}}_{\beta_{n}}^{n}(1)+\frac{2 \sigma_{n} \mathcal{R}_{1, \beta_{n}}^{n}(1)}{n^{1 / \alpha}}+\frac{2 \sigma_{n} \mathcal{R}_{\beta_{n}}^{n}(1)}{n^{1 / \alpha}}+2 \sigma_{n} \mathcal{R}_{9, \beta_{n}}^{n}(1) \widehat{\mathcal{H}}_{\beta_{n}}^{n}(1)+\frac{2 \sigma_{n}^{2} \mathcal{R}_{1, \beta_{n}}^{n}(1) \mathcal{R}_{9, \beta_{n}}^{n}(1)}{n^{1 / \alpha}}+\frac{2 \sigma_{n}^{2} \mathcal{R}_{\beta_{n}}^{n}(1) \mathcal{R}_{9, \beta_{n}}^{n}(1)}{n^{1 / \alpha}}
\end{array}\right),
\end{aligned}
$$

where $\mathrm{C}$ is some deterministic constant and $O\left(\frac{1}{n^{2 / \alpha}}\right)$ is a random variable bounded by $\frac{C}{n^{2 / \alpha}}$. From (3.16), (4.25), (4.26) and Lemma 5.3, we also conclude that

$$
\left(\begin{array}{c}
\frac{\sigma_{n}^{2}}{n^{2 / \alpha-1}} V_{1}^{n, \theta_{n}} \\
\frac{\sigma_{n}^{2}}{n^{1 / \alpha}} V_{1}^{n, \sigma_{n}}
\end{array}\right) \frac{2 \mathcal{H}_{\bar{Y}_{1}^{n, \beta_{n}, x_{0}}}(1)}{\left(U_{1}^{n, \beta_{n}}\right)} \frac{n \rightarrow \infty}{\mathbf{L}^{p}, \forall p \geq 1}\left(\begin{array}{c}
0 \\
2 \mathcal{H}_{L^{\alpha}}(1)
\end{array}\right)
$$

Term 3: From (3.13) and $\frac{W_{1}^{n, \beta_{n}}}{\left(U_{1}^{n, \beta_{n}}\right)^{2}}=\frac{n^{1 / \alpha}}{\sigma_{n}} \widehat{\mathcal{H}}_{1, \beta_{n}}^{n}(1)+\mathcal{R}_{2, \beta_{n}}^{n}(1)$ where $\widehat{\mathcal{H}}_{1, \beta_{n}}^{n}(1)$ and $\mathcal{R}_{2, \beta_{n}}^{n}(1)$ are given by (3.14) and (4.7), we have

$$
\begin{aligned}
& \left(\begin{array}{c}
\frac{\sigma_{n}^{2}}{n^{2 / \alpha-1}} \partial_{\theta} \bar{Y}_{1}^{n, \beta_{n}, x_{0}} \\
\frac{\sigma_{n}^{2}}{n^{1 / \alpha}} \partial_{\sigma} \bar{Y}_{1}^{n, \beta_{n}, x_{0}}
\end{array}\right) \frac{\mathcal{H}_{\bar{Y}_{1}^{n, \beta_{n}, x_{0}}}(1) W_{1}^{n, \beta_{n}}}{\left(U_{1}^{n, \beta_{n}}\right)^{2}} \\
& =\left(\begin{array}{c}
\frac{\sigma_{n}^{2}}{n^{2 / \alpha-1}} \partial_{\theta} \bar{Y}_{1}^{n, \beta_{n}, x_{0}} \\
\frac{\sigma_{n}^{2}}{n^{1 / \alpha}} \partial_{\sigma} \bar{Y}_{1}^{n, \beta_{n}, x_{0}}
\end{array}\right)\left[\frac{n^{1 / \alpha} \widehat{\mathcal{H}}_{\beta_{n}}^{n}(1)}{\sigma_{n}}+\mathcal{R}_{1, \beta_{n}}^{n}(1)+\mathcal{R}_{\beta_{n}}^{n}(1)\right]\left[\frac{n^{1 / \alpha}}{\sigma_{n}} \widehat{\mathcal{H}}_{1, \beta_{n}}^{n}(1)+\mathcal{R}_{2, \beta_{n}}^{n}(1)\right] \\
& =\left(\begin{array}{c}
n \partial_{\theta} \bar{Y}_{1}^{n, \beta_{n}, x_{0}} \\
n^{1 / \alpha} \partial_{\sigma} \bar{Y}_{1}^{n, \beta_{n}, x_{0}}
\end{array}\right) \widehat{\mathcal{H}}_{\beta_{n}}^{n}(1) \widehat{\mathcal{H}}_{1, \beta_{n}}^{n}(1)+\left(\begin{array}{c}
\frac{\sigma_{n}}{n^{1 / \alpha-1}} \partial_{\theta} \bar{Y}_{1}^{n, \beta_{n}, x_{0}} \\
\sigma_{n} \partial_{\sigma} \bar{Y}_{1}^{n, \beta_{n}, x_{0}}
\end{array}\right) \widehat{\mathcal{H}}_{\beta_{n}}^{n}(1) \mathcal{R}_{2, \beta_{n}}^{n}(1) \\
& +\left(\begin{array}{c}
\frac{\sigma_{n}^{2}}{n^{2 / \alpha-1}} \partial_{\theta} \bar{Y}_{1}^{n, \beta_{n}, x_{0}} \\
\frac{\sigma_{n}^{2}}{n^{1 / \alpha}} \partial_{\sigma} \bar{Y}_{1}^{n, \beta_{n}, x_{0}}
\end{array}\right) \mathcal{R}_{1, \beta_{n}}^{n}(1) \mathcal{R}_{2, \beta_{n}}^{n}(1)+\left(\begin{array}{c}
\frac{\sigma_{n}}{n^{1 / \alpha-1}} \partial_{\theta} \bar{Y}_{1}^{n, \beta_{n}, x_{0}} \\
\sigma_{n} \partial_{\sigma} \bar{Y}_{1}^{n, \beta_{n}, x_{0}}
\end{array}\right) \mathcal{R}_{1, \beta_{n}}^{n}(1) \widehat{\mathcal{H}}_{1, \beta_{n}}^{n}(1) \\
& +\left(\begin{array}{c}
\frac{\sigma_{n}}{n^{1 / \alpha-1}} \partial_{\theta} \bar{Y}_{1}^{n, \beta_{n}, x_{0}} \\
\sigma_{n} \partial_{\sigma} \bar{Y}_{1}^{n, \beta_{n}, x_{0}}
\end{array}\right) \mathcal{R}_{\beta_{n}}^{n}(1) \widehat{\mathcal{H}}_{1, \beta_{n}}^{n}(1)+\left(\begin{array}{c}
\frac{\sigma_{n}^{2}}{n^{2 / \alpha-1}} \partial_{\theta} \bar{Y}_{1}^{n, \beta_{n}, x_{0}} \\
\frac{\sigma_{n}^{2}}{n^{1 / \alpha}} \partial_{\sigma} \bar{Y}_{1}^{n, \beta_{n}, x_{0}}
\end{array}\right) \mathcal{R}_{\beta_{n}}^{n}(1) \mathcal{R}_{2, \beta_{n}}^{n}(1) .
\end{aligned}
$$

From (3.16), (4.25), (4.26), Lemmas 5.1 and 5.4, we also conclude that

$$
\left(\begin{array}{c}
\frac{\sigma_{n}^{2}}{n^{2 / \alpha-1}} \partial_{\theta} \bar{Y}_{1}^{n, \beta_{n}, x_{0}} \\
\frac{\sigma_{n}^{2}}{n^{1 / \alpha}} \partial_{\sigma} \bar{Y}_{1}^{n, \beta_{n}, x_{0}}
\end{array}\right) \frac{\mathcal{H}_{\bar{Y}_{1}^{n, \beta_{n}, x_{0}}}(1) W_{1}^{n, \beta_{n}}}{\left(U_{1}^{n, \beta_{n}}\right)^{2}} \underset{\mathbf{L}^{p}, \forall p \geq 1}{\stackrel{n \rightarrow \infty}{\longrightarrow}}\left(\begin{array}{c}
\partial_{\theta} b\left(x_{0}, \theta\right) \mathcal{H}_{1, L_{\alpha}}(1) \mathcal{H}_{L_{\alpha}}(1) \\
L_{1}^{\alpha} \mathcal{H}_{1, L_{\alpha}}(1) \mathcal{H}_{L_{\alpha}}(1) .
\end{array}\right)
$$


Term 4: Using $\frac{W_{1}^{n, \beta_{n}}}{\left(U_{1}^{n, \beta_{n}}\right)^{2}}=\frac{n^{1 / \alpha}}{\sigma_{n}} \widehat{\mathcal{H}}_{1, \beta_{n}}^{n}(1)+\mathcal{R}_{2, \beta_{n}}^{n}(1)$ again, we can rewrite

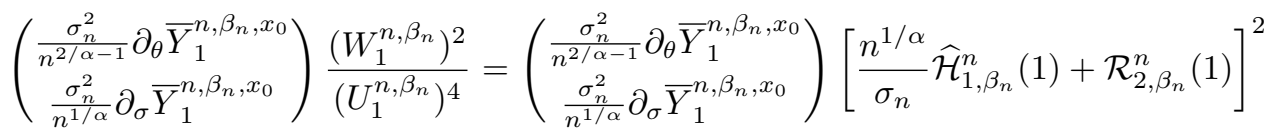

$$
\begin{aligned}
& =\left(\begin{array}{c}
n \partial_{\theta} \bar{Y}_{1}^{n, \beta_{n}, x_{0}} \\
n^{1 / \alpha} \partial_{\sigma} \bar{Y}_{1}^{n, \beta_{n}, x_{0}}
\end{array}\right)\left(\widehat{\mathcal{H}}_{1, \beta_{n}}^{n}(1)\right)^{2}+\left(\begin{array}{c}
\frac{\sigma_{n}^{2}}{n^{2 / \alpha-1}} \partial_{\theta} \bar{Y}_{1}^{n, \beta_{n}, x_{0}} \\
\frac{\sigma_{n}^{2}}{n^{1 / \alpha}} \partial_{\sigma} \bar{Y}_{1}^{n, \beta_{n}, x_{0}}
\end{array}\right)\left(\mathcal{R}_{2, \beta_{n}}^{n}(1)\right)^{2} \\
& +\left(\begin{array}{c}
\frac{2 \sigma_{n}}{n^{1 / \alpha-1}} \partial_{\theta} \bar{Y}_{1}^{n, \beta_{n}, x_{0}} \\
2 \sigma_{n} \partial_{\sigma} \bar{Y}_{1}^{n, \beta_{n}, x_{0}}
\end{array}\right) \mathcal{R}_{2, \beta_{n}}^{n}(1) \widehat{\mathcal{H}}_{1, \beta_{n}}^{n}(1) .
\end{aligned}
$$

From (3.16), (4.25), (4.26), Lemmas 5.4 and 5.1, we also conclude that

$$
\left(\begin{array}{c}
\frac{\sigma_{n}^{2}}{n^{2 / \alpha-1}} \partial_{\theta} \bar{Y}_{1}^{n, \beta_{n}, x_{0}} \\
\frac{\sigma_{n}^{2}}{n^{1 / \alpha}} \partial_{\sigma} \bar{Y}_{1}^{n, \beta_{n}, x_{0}}
\end{array}\right) \frac{\left(W_{1}^{n, \beta_{n}}\right)^{2}}{\left(U_{1}^{n, \beta_{n}}\right)^{4}} \underset{\mathbf{L}^{p}, \forall p \geq 1}{\stackrel{n \rightarrow \infty}{\longrightarrow}}\left(\begin{array}{c}
\partial_{\theta} b\left(x_{0}, \theta\right)\left(\mathcal{H}_{1, L^{\alpha}}(1)\right)^{2} \\
L_{1}^{\alpha}\left(\mathcal{H}_{1, L^{\alpha}}(1)\right)^{2}
\end{array}\right)
$$

Term 5: From Lemma 5.3 we can estimate the fifth term as

$$
\begin{aligned}
\left(\begin{array}{c}
\frac{\sigma_{n}^{2}}{n^{2 / \alpha-1}} V_{1}^{n, \theta_{n}} \\
\frac{\sigma_{n}^{2}}{n^{1 / \alpha}} V_{1}^{n, \sigma_{n}}
\end{array}\right) \frac{W_{1}^{n, \beta_{n}}}{\left(U_{1}^{n, \beta_{n}}\right)^{3}} & =\left(\begin{array}{c}
O\left(\frac{1}{n^{2 / \alpha}}\right) \\
\frac{\sigma_{n}^{2}}{n^{1 / \alpha}}\left(\mathcal{R}_{9, \beta_{n}}^{n}(1)+\frac{1}{\sigma_{n}}\right)
\end{array}\right)\left(\frac{n^{1 / \alpha}}{\sigma_{n}} \widehat{\mathcal{H}}_{1, \beta_{n}}^{n}(1)+\mathcal{R}_{2, \beta_{n}}^{n}(1)\right) \\
& =\left(\begin{array}{c}
O\left(\frac{1}{n^{1 / \alpha}}\right) \widehat{\mathcal{H}}_{1, \beta_{n}}^{n}(1)+O\left(\frac{1}{n^{2 / \alpha}}\right) \mathcal{R}_{2, \beta_{n}}^{n}(1) \\
\sigma_{n} \mathcal{R}_{9, \beta_{n}}^{n}(1) \widehat{\mathcal{H}}_{1, \beta_{n}}^{n}(1)+\frac{\sigma_{n}^{2}}{n^{1 / \alpha}} \mathcal{R}_{9, \beta_{n}}^{n}(1) \mathcal{R}_{2, \beta_{n}}^{n}(1)+\widehat{\mathcal{H}}_{1, \beta_{n}}^{n}(1)+\frac{\sigma_{n}}{n^{1 / \alpha}} \mathcal{R}_{2, \beta_{n}}^{n}(1)
\end{array}\right)
\end{aligned}
$$

where $C$ is some deterministic constant. From (3.16), (4.25), Lemma 5.3, we also conclude that

$$
\left(\begin{array}{c}
\frac{\sigma_{n}^{2}}{n^{2 / \alpha-1}} V_{1}^{n, \theta_{n}} \\
\frac{\sigma_{n}^{2}}{n^{1 / \alpha}} V_{1}^{n, \sigma_{n}}
\end{array}\right) \frac{W_{1}^{n, \beta_{n}}}{\left(U_{1}^{n, \beta_{n}}\right)^{3}} \underset{\mathbf{L}^{p}, \forall p \geq 1}{\stackrel{n \rightarrow \infty}{\longrightarrow}}\left(\begin{array}{c}
0 \\
\mathcal{H}_{1, L^{\alpha}}(1)
\end{array}\right)
$$

Term 6: Using Lemma 5.2 we write the sixth term as

$$
\begin{aligned}
\left(\begin{array}{c}
\frac{\sigma_{n}^{2}}{n^{2 / \alpha-1}} \partial_{\theta} \bar{Y}_{1}^{n, \beta_{n}, x_{0}} \\
\frac{\sigma_{n}^{2}}{n^{1 / \alpha}} \partial_{\sigma} \bar{Y}_{1}^{n, \beta_{n}, x_{0}}
\end{array}\right) \frac{D_{1}^{n, \beta_{n}}}{\left(U_{1}^{n, \beta_{n}}\right)^{2}}= & \left(\begin{array}{c}
\frac{n}{2} \partial_{\theta} \bar{Y}_{1}^{n, \beta_{n}, x_{0}} \\
\frac{n^{1 / \alpha}}{2} \partial_{\sigma} \bar{Y}_{1}^{n, \beta_{n}, x_{0}}
\end{array}\right) \widehat{\mathcal{H}}_{3, \beta_{n}}^{n}(1)+\left(\begin{array}{c}
\frac{\sigma_{n}^{2}}{n^{1 / \alpha-1}} \partial_{\theta} \bar{Y}_{1}^{n, \beta_{n}, x_{0}} \\
\sigma_{n}^{2} \partial_{\sigma} \bar{Y}_{1}^{n, \beta_{n}, x_{0}}
\end{array}\right) \\
& \times\left(\mathcal{R}_{4, \beta_{n}}^{n}(1)+\mathcal{R}_{5, \beta_{n}}^{n}(1)+\mathcal{R}_{6, \beta_{n}}^{n}(1)\right) .
\end{aligned}
$$

Applying Lemma 5.2, Lemma 5.5 and Lemma 5.1 we obtain that

$$
\left(\begin{array}{c}
\frac{\sigma_{n}^{2}}{n^{2 / \alpha-1}} \partial_{\theta} \bar{Y}_{1}^{n, \beta_{n}, x_{0}} \\
\frac{\sigma_{n}^{2}}{n^{1 / \alpha}} \partial_{\sigma} \bar{Y}_{1}^{n, \beta_{n}, x_{0}}
\end{array}\right) \frac{2 D_{1}^{n, \beta_{n}}}{\left(U_{1}^{n, \beta_{n}}\right)^{2}} \frac{n \rightarrow \infty}{\mathbf{L}^{p}, \forall p \geq 1}\left(\begin{array}{c}
\partial_{\theta} b\left(x_{0}, \theta\right) \mathcal{H}_{3, L^{\alpha}}(1) \\
L_{1}^{\alpha} \mathcal{H}_{3, L^{\alpha}}(1)
\end{array}\right)
$$

where $\mathcal{H}_{3, L^{\alpha}}(1)$ is defined in Lemma 5.2.

Term 7: From Lemma 5.2, we can rewrite the seventh term as 


$$
\left(\begin{array}{c}
\frac{\sigma_{n}^{2}}{n^{2 / \alpha-1}} \partial_{\theta} \bar{Y}_{1}^{n, \beta_{n}, x_{0}} \\
\frac{\sigma_{n}^{2}}{n^{1 / \alpha}} \partial_{\sigma} \bar{Y}_{1}^{n, \beta_{n}, x_{0}}
\end{array}\right) \frac{Q_{1}^{n, \beta_{n}}}{\left(U_{1}^{n, \beta_{n}}\right)^{3}}=\left(\begin{array}{c}
n \partial_{\theta} \bar{Y}_{1}^{n, \beta_{n}, x_{0}} \\
n^{1 / \alpha} \partial_{\sigma} \bar{Y}_{1}^{n, \beta_{n}, x_{0}}
\end{array}\right) \widehat{\mathcal{H}}_{4, \beta_{n}}^{n}(1)+\left(\begin{array}{c}
\frac{\sigma_{n}^{2}}{n^{1 / \alpha-1}} \partial_{\theta} \bar{Y}_{1}^{n, \beta_{n}, x_{0}} \\
\sigma_{n}^{2} \partial_{\sigma} \bar{Y}_{1}^{n, \beta_{n}, x_{0}}
\end{array}\right)\left(\mathcal{R}_{7, \beta_{n}}^{n}(1)+\mathcal{R}_{8, \beta_{n}}^{n}(1)\right)
$$

Applying Lemmas 5.2, 5.5 and 5.1 we obtain that

$$
\left(\begin{array}{c}
\frac{\sigma_{n}^{2}}{n^{2 / \alpha-1}} \partial_{\theta} \bar{Y}_{1}^{n, \beta_{n}, x_{0}} \\
\frac{\sigma_{n}^{2}}{n^{1 / \alpha}} \partial_{\sigma} \bar{Y}_{1}^{n, \beta_{n}, x_{0}}
\end{array}\right) \frac{Q_{1}^{n, \beta_{n}}}{\left(U_{1}^{n, \beta_{n}}\right)^{3}} \frac{n \rightarrow \infty}{\mathbf{L}^{p}, \forall p \geq 1}\left(\begin{array}{c}
\partial_{\theta} b\left(x_{0}, \theta\right) \mathcal{H}_{4, L^{\alpha}}(1) \\
L_{1}^{\alpha} \mathcal{H}_{4, L^{\alpha}}(1)
\end{array}\right)
$$

where $\mathcal{H}_{4, L^{\alpha}}(1)$ is defined in Lemma 5.2 .

Term 8: From Lemma 5.3, we have

$$
\left(\begin{array}{c}
\frac{\sigma_{n}^{2}}{n^{2 / \alpha-1}} T_{1}^{n, \theta_{n}} \\
\frac{\sigma_{n}^{2}}{n^{1 / \alpha}} T_{1}^{n, \sigma_{n}}
\end{array}\right) \frac{1}{\left(U_{1}^{n, \beta_{n}}\right)^{2}}=\left(\begin{array}{c}
\frac{\sigma_{n}^{2}}{n^{2 / \alpha-1}} \frac{T_{1}^{n, \theta_{n}}}{\left(U_{1}^{n, \beta_{n}}\right)^{2}} \\
\widehat{\mathcal{H}}_{5, \beta_{n}}^{n}(1)+\sigma_{n}^{2} \mathcal{R}_{10, \beta_{n}}^{n}(1)+\sigma_{n}^{2} \mathcal{R}_{11, \beta_{n}}^{n}(1)+\sigma_{n}^{2} \mathcal{R}_{12, \beta_{n}}^{n}(1)
\end{array}\right)
$$

Using the results of Lemma 5.3, we easily deduce that

$$
\left(\begin{array}{c}
\frac{\sigma_{n}^{2}}{n^{2 / \alpha-1}} T_{1}^{n, \theta_{n}} \\
\frac{\sigma_{n}^{2}}{n^{1 / \alpha}} T_{1}^{n, \sigma_{n}}
\end{array}\right) \frac{1}{\left(U_{1}^{n, \beta_{n}}\right)^{2}} \underset{\mathbf{L}^{p}, \forall p \geq 1}{\stackrel{n \rightarrow \infty}{\longrightarrow}}\left(\begin{array}{c}
0 \\
\mathcal{H}_{5, L^{\alpha}}(1)
\end{array}\right)
$$

where $\mathcal{H}_{5, L^{\alpha}}(1)$ is defined in Lemma 5.3 .

Finally from the above convergences, we can deduce the result of Proposition 5.7.

\subsection{Proof of Theorem 2.5}

We will first prove part $i i$ ) and then give a proof for part $i$ ).

ii) Remark that from (5.24), (5.25)

$$
\sup _{u \in \mathbb{R}} \sup _{n} \mathbb{E}\left|1_{\left\{\bar{Y}_{1}^{n, \beta_{n}, x_{0}} \geq \frac{u \sigma_{n}}{n^{1 / \alpha}}+\varsigma_{1}^{n, \theta_{n}, x_{0}}\right\}} \frac{\sigma_{n}^{2}}{n^{2 / \alpha-1}} \mathcal{H}_{\bar{Y}_{1}^{n, \beta_{n}, x_{0}}}\left(\mathcal{H}_{\bar{Y}_{1}^{n, \beta_{n}, x_{0}}}\left(\partial_{\theta} \bar{Y}_{1}^{n, \beta_{n}, x_{0}}\right)\right)\right|<\infty
$$

and

$$
\sup _{u \in \mathbb{R}} \sup _{n} \mathbb{E}\left|1_{\left\{\bar{Y}_{1}^{n, \beta_{n}, x_{0}} \geq \frac{u \sigma_{n}}{n^{1 / \alpha}}+\varsigma_{1}^{n, \theta_{n}, x_{0}}\right\}} \frac{\sigma_{n}^{2}}{n^{1 / \alpha}} \mathcal{H}_{\bar{Y}_{1}^{n, \beta_{n}, x_{0}}}\left(\mathcal{H}_{\bar{Y}_{1}^{n, \beta_{n}, x_{0}}}\left(\partial_{\sigma} \bar{Y}_{1}^{n, \beta_{n}, x_{0}}\right)\right)\right|<\infty
$$

By representation (5.1) this leads to

$$
\sup _{u \in \mathbb{R}} \sup _{n}\left|\frac{\sigma_{n}^{2}}{n^{\frac{2}{\alpha}-1}} \partial_{\theta} p_{\frac{1}{n}}^{\beta_{n}}\left(x_{0}, \frac{u \sigma_{n}}{n^{1 / \alpha}}+\varsigma_{1}^{n, \theta_{n}, x_{0}}\right)\right|<\infty \text { and } \sup _{u \in \mathbb{R}} \sup _{n}\left|\frac{\sigma_{n}^{2}}{n^{1 / \alpha}} \partial_{\sigma} p_{\frac{1}{n}}^{\beta_{n}}\left(x_{0}, \frac{u \sigma_{n}}{n^{1 / \alpha}}+\varsigma_{1}^{n, \theta_{n}, x_{0}}\right)\right|<\infty
$$

i) From (5.1), (4.27) and Proposition 5.7, we easily deduce that

$$
\frac{\sigma_{n}^{2}}{n^{1 / \alpha}} \partial_{\sigma} p_{\frac{1}{n}}^{\beta_{n}}\left(x_{0}, \frac{u \sigma_{n}}{n^{1 / \alpha}}+\varsigma_{1}^{n, \theta_{n}, x_{0}}\right)=\frac{\sigma_{n}^{2}}{n^{1 / \alpha}} \partial_{\sigma} q^{n, \beta_{n}, x_{0}}\left(\frac{u \sigma_{n}}{n^{1 / \alpha}}+\varsigma_{1}^{n, \theta_{n}, x_{0}}\right) \stackrel{n \rightarrow \infty}{\longrightarrow} \mathbb{E}\left[1_{[u, \infty)}\left(L_{1}^{\alpha}\right) \mathcal{H}^{(2)}\right],
$$




$$
\frac{\sigma_{n}^{2}}{n^{\frac{2}{\alpha}-1}} \partial_{\theta} p_{\frac{1}{n}}^{\beta_{n}}\left(x_{0}, \frac{u \sigma_{n}}{n^{1 / \alpha}}+\varsigma_{1}^{n, \theta_{n}, x_{0}}\right)=\frac{\sigma_{n}^{2}}{n^{\frac{2}{\alpha}-1}} \partial_{\theta} q^{n, \beta_{n}, x_{0}}\left(\frac{u \sigma_{n}}{n^{1 / \alpha}}+\varsigma_{1}^{n, \theta_{n}, x_{0}}\right) \stackrel{n \rightarrow \infty}{\longrightarrow} \partial_{\theta} b\left(x_{0}, \theta\right) \times \mathbb{E}\left[1_{[u, \infty)}\left(L_{1}^{\alpha}\right) \mathcal{H}_{1}^{(2)}\right],
$$

where $\mathcal{H}^{(2)}$ and $\mathcal{H}_{1}^{(2)}$ are defined in Proposition 5.7.

To finish the proof of Theorem 2.5, it remains to show that $\mathbb{E}\left[1_{[u, \infty)}\left(L_{1}^{\alpha}\right) \mathcal{H}_{1}^{(2)}\right]=-\varphi_{\alpha}^{\prime}(u)$ and $\mathbb{E}\left[1_{[u, \infty)}\left(L_{1}^{\alpha}\right) \mathcal{H}^{(2)}\right]=$ $-\left[\varphi_{\alpha}(u)+u \varphi_{\alpha}{ }^{\prime}(u)\right]$. This is done in Lemma 5.8 below.

Lemma 5.8. We have

$$
\begin{aligned}
\varphi_{\alpha}^{\prime}(u) & =-\mathbb{E}\left[1_{[u, \infty)}\left(L_{1}^{\alpha}\right) \mathcal{H}_{1}^{(2)}\right], \\
-\left[\varphi_{\alpha}(u)+u \varphi_{\alpha}^{\prime}(u)\right] & =\mathbb{E}\left[1_{[u, \infty)}\left(L_{1}^{\alpha}\right) \mathcal{H}^{(2)}\right],
\end{aligned}
$$

where $\varphi_{\alpha}$ is the density of $L_{1}^{\alpha}$ and $\mathcal{H}^{(2)}$ and $\mathcal{H}_{1}^{(2)}$ are defined in Proposition 5.7.

Proof. Let us consider the situation where $b(x, \theta)=\theta$ and $x_{0}=0$. In that case, we have ${\overline{Y_{1}}}^{n, \beta, x_{0}}=\frac{\theta}{n}+\frac{\sigma}{n^{1 / \alpha}} L_{1}^{n}$ and thus the density of ${\overline{Y_{1}}}^{n, \beta, x_{0}}$ is related to the density of $L_{1}^{n}$ by the relation,

$$
q^{n, \beta, x_{0}}(u)=\frac{n^{1 / \alpha}}{\sigma} \varphi^{n}\left(\frac{n^{1 / \alpha}}{\sigma}\left(u-\frac{\theta}{n}\right)\right)
$$

Then,

$$
\begin{aligned}
& \partial_{\theta} q^{n, \beta, x_{0}}(u)=-\frac{n^{2 / \alpha-1}}{\sigma^{2}}\left(\varphi^{n}\right)^{\prime}\left(\frac{n^{1 / \alpha}}{\sigma}\left(u-\frac{\theta}{n}\right)\right), \\
& \partial_{\sigma} q^{n, \beta, x_{0}}(u)=-\frac{n^{1 / \alpha}}{\sigma^{2}} \varphi^{n}\left(\frac{n^{1 / \alpha}}{\sigma}\left(u-\frac{\theta}{n}\right)\right)-\frac{\left(n^{1 / \alpha}\right)^{2}}{\sigma^{3}}\left(u-\frac{\theta}{n}\right)\left(\varphi^{n}\right)^{\prime}\left(\frac{n^{1 / \alpha}}{\sigma}\left(u-\frac{\theta}{n}\right)\right),
\end{aligned}
$$

By a change of variables, we get

$$
\begin{aligned}
& \partial_{\theta} q^{n, \beta, x_{0}}\left(\frac{u \sigma}{n^{1 / \alpha}}+\frac{\theta}{n}\right)=-\frac{n^{2 / \alpha-1}}{\sigma^{2}}\left(\varphi^{n}\right)^{\prime}(u) \\
& \partial_{\sigma} q^{n, \beta, x_{0}}\left(\frac{u \sigma}{n^{1 / \alpha}}+\frac{\theta}{n}\right)=-\frac{n^{1 / \alpha}}{\sigma^{2}}\left[\varphi^{n}(u)+u\left(\varphi^{n}\right)^{\prime}(u)\right] .
\end{aligned}
$$

Hence, we can apply the results of part $i$ ) of Theorem 2.5 and (5.28), (5.29) in this specific setting. This yields

$$
\begin{aligned}
& \forall u, \quad\left(\varphi^{n}\right)^{\prime}(u) \stackrel{n \rightarrow \infty}{\longrightarrow}-\mathbb{E}\left[1_{[u, \infty)}\left(L_{1}^{\alpha}\right) \mathcal{H}_{1}^{(2)}\right], \\
& \forall u, \quad\left[\varphi^{n}(u)+u\left(\varphi^{n}\right)^{\prime}(u)\right] \stackrel{n \rightarrow \infty}{\longrightarrow}-\mathbb{E}\left[1_{[u, \infty)}\left(L_{1}^{\alpha}\right) \mathcal{H}^{(2)}\right], \\
& \sup _{u, n}\left|\left(\varphi^{n}\right)^{\prime}(u)\right|<\infty, \\
& \sup _{u, n}\left|\varphi^{n}(u)+u\left(\varphi^{n}\right)^{\prime}(u)\right|<\infty .
\end{aligned}
$$

Let us denote $\mathcal{X}(u)=-\mathbb{E}\left[1_{[u, \infty)}\left(L_{1}^{\alpha}\right) \mathcal{H}_{1}^{(2)}\right]$ and assume by contradiction that $\mathcal{X} \neq \varphi_{\alpha}^{\prime}$. Using the continuity of $u \mapsto \mathcal{X}(u)$, there exists a smooth, compactly supported function $f$, such that $\int \mathcal{X}(u) f(u) \mathrm{d} u \neq \int \varphi_{\alpha}^{\prime}(u) f(u) \mathrm{d} u$. 
Now, on the one hand we have

$$
\int\left(\varphi^{n}\right)^{\prime}(u) f(u) \mathrm{d} u \stackrel{n \rightarrow \infty}{\longrightarrow} \int \mathcal{X}(u) f(u) \mathrm{d} u
$$

where we have used the dominated convergence theorem, together with (5.30), (5.32).

On the other hand, we can write,

$$
\begin{aligned}
\int\left(\varphi^{n}\right)^{\prime}(u) f(u) \mathrm{d} u & =-\int \varphi^{n}(u) f^{\prime}(u) \mathrm{d} u \\
& =-\mathbb{E}\left[f^{\prime}\left(L_{1}^{n}\right)\right] \stackrel{n \rightarrow \infty}{\longrightarrow}-\mathbb{E}\left[f^{\prime}\left(L_{1}^{\alpha}\right)\right] \\
& =-\int \varphi_{\alpha}(u) f^{\prime}(u) \mathrm{d} u=\int \varphi_{\alpha}^{\prime}(u) f(u) \mathrm{d} u,
\end{aligned}
$$

where the convergence (5.35) is obtained in the same way as (4.35). Clearly (5.36) contradicts (5.34), and we get $\mathbb{E}\left[1_{[u, \infty)}\left(L_{1}^{\alpha}\right) \mathcal{H}_{1}^{(2)}\right]=-\varphi_{\alpha}^{\prime}(u)$.

By the same method, let us denote $\mathcal{X}_{1}(u)=-\mathbb{E}\left[1_{[u, \infty)}\left(L_{1}^{\alpha}\right) \mathcal{H}^{(2)}\right]$ and assume by contradiction that $u \mapsto \mathcal{X}_{1}(u)$ is different from $u \mapsto\left[\varphi_{\alpha}(u)+u\left(\varphi_{\alpha}\right)^{\prime}(u)\right]$. Using the continuity of $u \mapsto \mathcal{X}_{1}(u)$, there exists a smooth, compactly supported function $f$, such that $\int \mathcal{X}_{1}(u) f(u) \mathrm{d} u \neq \int\left[\varphi_{\alpha}(u)+u\left(\varphi_{\alpha}\right)^{\prime}(u)\right] f(u) \mathrm{d} u$. Now, we have

$$
\int\left[\varphi^{n}(u)+u\left(\varphi^{n}\right)^{\prime}(u)\right] f(u) \mathrm{d} u \stackrel{n \rightarrow \infty}{\longrightarrow} \int \mathcal{X}_{1}(u) f(u) \mathrm{d} u,
$$

where we have used the dominated convergence theorem, together with (5.31), (5.33).

On the other hand, letting $g(u)=u f(u)$ and using the integration by parts formula, we can write,

$$
\begin{aligned}
& \int {\left[\varphi^{n}(u)+u\left(\varphi^{n}\right)^{\prime}(u)\right] f(u) \mathrm{d} u } \\
&= \int \varphi^{n}(u) f(u) \mathrm{d} u+\int\left(\varphi^{n}\right)^{\prime}(u) g(u) \mathrm{d} u \\
&= \mathbb{E}\left[f\left(L_{1}^{n}\right)\right]-\int \varphi^{n}(u) g^{\prime}(u) \mathrm{d} u=\mathbb{E}\left[f\left(L_{1}^{n}\right)\right]-\mathbb{E}\left[g^{\prime}\left(L_{1}^{n}\right)\right] \stackrel{n \rightarrow \infty}{\longrightarrow} \mathbb{E}\left[f\left(L_{1}^{\alpha}\right)\right]-\mathbb{E}\left[g^{\prime}\left(L_{1}^{\alpha}\right)\right] \\
&=\int \varphi_{\alpha}(u) f(u) \mathrm{d} u-\int \varphi_{\alpha}(u) g^{\prime}(u) \mathrm{d} u=\int \varphi_{\alpha}(u) f(u) \mathrm{d} u+\int \varphi_{\alpha}^{\prime}(u) g(u) \mathrm{d} u,
\end{aligned}
$$

where the convergence (5.38) is obtained in the same way as (4.35). Clearly (5.39) contradicts (5.37), and the lemma is proved.

\subsection{Proofs of the intermediate lemmas}

In this subsection, we give the proofs of Lemmas 5.1-5.5 of Section 5.2.

Proof of Lemma 5.1: i) Since $b$ has bounded derivatives, we obtain from (5.3)

$$
\sup _{\beta \in Q}\left|\partial_{\theta} \bar{Y}_{1}^{n, \beta, x_{0}}\right| \leq \frac{C}{n}
$$


ii) From (5.4) and Gronwall's Lemma, we get

$$
\begin{aligned}
\sup _{\beta \in Q} \sup _{s \in[0,1]}\left|\partial_{\sigma} \bar{Y}_{s}^{n, \beta, x_{0}}\right| & \leq \frac{C}{n^{1 / \alpha}} \sup _{s \in[0,1]}\left|L_{s}^{n}\right| \\
& \leq \frac{C}{n^{1 / \alpha}} \sup _{s \in[0,1]}\left|\int_{0}^{s} \int_{|z| \leq 1} z \tilde{\mu}(\mathrm{d} u, \mathrm{~d} z)\right|+\frac{C}{n^{1 / \alpha}} \int_{0}^{1} \int_{|z|>1}|z| \mu^{(n)}(\mathrm{d} u, \mathrm{~d} z)
\end{aligned}
$$

We now consider the first term of (5.40).

Using Kunita's first inequality (see Thm. 4.4.23 in [1]) $\forall p \geq 2$, we have $\mathbb{E} \sup _{s \in[0,1]}\left|\int_{0}^{s} \int_{|z| \leq 1} z \tilde{\mu}(\mathrm{d} u, \mathrm{~d} z)\right|^{p}<\infty$, and the first term of (5.40) converges to zero in $\mathbf{L}^{p}, \forall p \geq 1$.

We now consider the second term of (5.40).

From $\mu^{(n)}(\mathrm{d} s, \mathrm{~d} z)=\tilde{\mu}^{(n)}(\mathrm{d} s, \mathrm{~d} z)+v^{(n)}(\mathrm{d} s, \mathrm{~d} z)$ then for $C_{2}(p)$ a positive constant, we have

$$
\frac{1}{n^{p / \alpha}} \mathbb{E}\left(\int_{0}^{1} \int_{|z|>1}|z| \mu^{(n)}(\mathrm{d} u, \mathrm{~d} z)\right)^{p} \leq \frac{C_{2}(p)}{n^{p / \alpha}}\left[\mathbb{E}\left(\int_{0}^{1} \int_{|z|>1}|z| \tilde{\mu}^{(n)}(\mathrm{d} u, \mathrm{~d} z)\right)^{p}+\mathbb{E}\left(\int_{0}^{1} \int_{|z|>1}|z| v^{(n)}(\mathrm{d} u, \mathrm{~d} z)\right)^{p}\right] .
$$

Using again Kunita's first inequality (see Thm. 4.4.23 in [1]), for $p \geq 2$, there exists a positive constant $C_{3}(p)$ such that

$$
\begin{aligned}
& \frac{1}{n^{p / \alpha}} \mathbb{E}\left[\left|\int_{0}^{1} \int_{|z|>1}\right| z\left|\tilde{\mu}^{(n)}(\mathrm{d} s, \mathrm{~d} z)\right|\right]^{p} \\
& \leq \frac{C_{3}(p)}{n^{p / \alpha}}\left[\left(\int_{0}^{1} \int_{|z|>1} z^{2} v^{(n)}(\mathrm{d} s, \mathrm{~d} z)\right)^{p / 2}+\left(\int_{0}^{1} \int_{|z|>1}|z|^{p} v^{(n)}(\mathrm{d} s, \mathrm{~d} z)\right)\right] \\
& =\frac{C_{3}(p)}{n^{p / \alpha}}\left(\int_{0}^{1} \int_{|z|>1} z^{2} \frac{1}{|z|^{1+\alpha}} \tau\left(z / n^{1 / \alpha}\right) \mathrm{d} z \mathrm{~d} s\right)^{p / 2}+\frac{C_{3}(p)}{n^{p / \alpha}}\left[\int_{0}^{1} \int_{|z|>1}|z|^{p} \frac{1}{|z|^{1+\alpha}} \tau\left(z / n^{1 / \alpha}\right) \mathrm{d} z \mathrm{~d} s\right] \\
& \leq \frac{2 C_{3}(p)}{n^{p / \alpha}}\left[\int_{0}^{1} \int_{1}^{2 n^{1 / \alpha}} \frac{1}{z^{\alpha-1}} \mathrm{~d} z \mathrm{~d} s\right]^{p / 2}+\left[\frac{2 C_{3}(p)}{n^{p / \alpha}} \int_{0}^{1} \int_{1}^{2 n^{1 / \alpha}} \frac{1}{z^{\alpha+1-p}} \mathrm{~d} z \mathrm{~d} s\right] \stackrel{n \rightarrow \infty}{\longrightarrow} 0,
\end{aligned}
$$

where we used that $0 \leq \tau \leq 1$ and $\tau=0$ on $[-2,2]^{c}$. Hence, we get that the second term of (5.40) also converges to zero in $\mathbf{L}^{p}, \forall p \geq 1$. This finishes the proof of $\left.i i\right)$.

Proof of Lemma 5.2: Recall that $D_{1}^{n, \beta}$ and $U_{1}^{n, \beta}$ are given by (5.7) and (4.3). The part $i$ ) is proved by decomposing $\frac{D_{1}^{n, \beta}}{\left(U_{1}^{n, \beta}\right)^{2}}$, then we obtain that the main term is (5.11) and the remainder terms are

$$
\begin{aligned}
\mathcal{R}_{4, \beta}^{n}(1)= & \frac{\int_{0}^{1} \int_{|z|>2}\left(\epsilon_{s}^{n}\right)^{-2} \rho^{n}(z) \rho^{n \prime}(z) \frac{\tau^{\prime}\left(z / n^{1 / \alpha}\right)}{\tau\left(z / n^{1 / \alpha}\right)} \mu^{(n)}(\mathrm{d} s, \mathrm{~d} z)}{2 \sigma^{2} n^{1 / \alpha}\left(\epsilon_{1}^{n}\right)^{2}\left(\int_{0}^{1} \int_{\mathbb{R}}\left(\epsilon_{s}^{n}\right)^{-2} \rho^{n}(z) \mu^{(n)}(\mathrm{d} s, \mathrm{~d} z)\right)^{2}}+ \\
& +\frac{\int_{0}^{1} \int_{|z|>2}\left(\epsilon_{s}^{n}\right)^{-2}\left(\rho^{n}(z)\right)^{2}\left[\frac{\tau^{\prime \prime}\left(z / n^{1 / \alpha}\right)}{\tau\left(z / n^{1 / \alpha}\right)}-\left(\frac{\tau^{\prime}\left(z / n^{1 / \alpha}\right)}{\tau\left(z / n^{1 / \alpha}\right)}\right)^{2}\right] \mu^{(n)}(\mathrm{d} s, \mathrm{~d} z)}{2 \sigma^{2} n^{1 / \alpha}\left(\epsilon_{1}^{n}\right)^{2}\left(\int_{0}^{1} \int_{\mathbb{R}}\left(\epsilon_{s}^{n}\right)^{-2} \rho^{n}(z) \mu^{(n)}(\mathrm{d} s, \mathrm{~d} z)\right)^{2}},
\end{aligned}
$$




$$
\begin{aligned}
\mathcal{R}_{5, \beta}^{n}(1) & =\frac{\left(\epsilon_{1}^{n}\right)^{2} \int_{0}^{1}\left(\epsilon_{s}^{n}\right)^{-2} b^{\prime \prime}\left(\bar{Y}_{s}^{n, \beta, x_{0}}, \theta\right)\left(2 L \bar{Y}_{s}^{n, \beta, x_{0}} U_{s}^{n, \beta}+W_{s}^{n, \beta}\right) \mathrm{d} s}{2 n^{1+1 / \alpha}\left(U_{1}^{n, \beta}\right)^{2}}, \\
\mathcal{R}_{6, \beta}^{n}(1) & =\frac{\left(\epsilon_{1}^{n}\right)^{2} \int_{0}^{1}\left(\epsilon_{s}^{n}\right)^{-2} b^{\prime \prime \prime}\left(\bar{Y}_{s}^{n, \beta, x_{0}}, \theta\right)\left(U_{s}^{n, \beta}\right)^{2} \mathrm{~d} s}{2 n^{1+1 / \alpha}\left(U_{1}^{n, \beta}\right)^{2}} .
\end{aligned}
$$

The part $i i)$ is proved by decomposing $\frac{Q_{1}^{n, \beta}}{\left(U_{1}^{n, \beta}\right)^{3}}$, then we obtain that the main term is (5.12) and the remainder terms are

$$
\begin{aligned}
& \mathcal{R}_{7, \beta}^{n}(1)=\frac{7\left(\epsilon_{1}^{n}\right)^{4} \int_{0}^{1}\left(\epsilon_{1}^{n}\right)^{-4} b^{\prime \prime}\left(\bar{Y}_{s}^{n, \beta, x_{0}}, \theta\right) W_{s}^{n, \beta} U_{s}^{n, \beta} \mathrm{d} s}{n^{1+1 / \alpha}\left(U_{1}^{n, \beta}\right)^{3}} \\
& \mathcal{R}_{8, \beta}^{n}(1)=\frac{2\left(\epsilon_{1}^{n}\right)^{4} \int_{0}^{1}\left(\epsilon_{1}^{n}\right)^{-4} b^{\prime \prime \prime}\left(\left(\bar{Y}_{s}^{n, \beta, x_{0}}, \theta\right)\left(U_{s}^{n, \beta}\right)^{3} \mathrm{~d} s\right.}{n^{1+1 / \alpha}\left(U_{1}^{n, \beta}\right)^{3}} .
\end{aligned}
$$

We now study the convergence of the main terms.

From (3.10), the boundedness of $b^{\prime}$ and Lemma 3.1, it is clear that $\widehat{\mathcal{H}}_{3, \beta_{n}}^{n}(1)$ converges almost surely to $\mathcal{H}_{3, L^{\alpha}}(1)$. Moreover, using again the boundedness of $b^{\prime}$, the upper and lower bounds of $\left(\epsilon_{s}^{n}\right)_{s \in[0,1]}$ and the fact that $\rho^{n}(z)$ is a non negative function, we deduce the upper bound, for some constant $C>0$,

$$
\left|\widehat{\mathcal{H}}_{3, \beta}^{n}(1)\right| \leq C\left[\frac{\int_{0}^{1} \int_{\mathbb{R}}\left(\rho^{n}(z)\left|\left(\rho^{n}\right)^{\prime \prime}(z)\right|+\rho^{n}(z)\left|\rho^{n \prime}(z)\right| \frac{(1+\alpha)}{|z|}+\left(\rho^{n}(z)\right)^{2} \frac{(1+\alpha)}{z^{2}}\right) \mu^{(n)}(\mathrm{d} s, \mathrm{~d} z)}{\left(\int_{0}^{1} \int_{\mathbb{R}} \rho^{n}(z) \mu^{(n)}(\mathrm{d} s, \mathrm{~d} z)\right)^{2}}\right] .
$$

Proceeding as in Step 1 in the proof of Theorem 2.2, we show that $\sup _{n}\left|\widehat{\mathcal{H}}_{3, \beta_{n}}^{n}(1)\right|^{p}$ is integrable. Then applying the dominated convergence theorem, we get $\widehat{\mathcal{H}}_{3, \beta_{n}}^{n}(1) \underset{\mathbf{L}^{p}, \forall p \geq 1}{\stackrel{n \rightarrow \infty}{\longrightarrow}} \mathcal{H}_{3, L^{\alpha}}(1)$. In the same way we prove that $\widehat{\mathcal{H}}_{4, \beta_{n}}^{n}(1) \underset{\mathbf{L}^{p}, \forall p \geq 1}{\stackrel{n \rightarrow \infty}{\longrightarrow}} \mathcal{H}_{4, L^{\alpha}}(1)$.

Turning to the remainder terms, since $b$ has bounded derivatives and $\sup _{\beta} \sup _{s} \frac{U_{s}^{n, \beta}}{U_{1}^{n, \beta}}$ is bounded, we obtain

$$
\begin{aligned}
& \sup _{\beta \in Q}\left|\mathcal{R}_{5, \beta}^{n}(1)\right| \leq C \sup _{\beta \in Q}\left[\frac{\sup _{s \in[0,1]}\left|L \bar{Y}_{s}^{n, \beta, x_{0}}\right|}{n^{1+1 / \alpha} U_{1}^{n, \beta}}+\frac{\sup _{s \in[0,1]}\left|W_{s}^{n, \beta}\right|}{n^{1+1 / \alpha}\left(U_{1}^{n, \beta}\right)^{2}}\right], \quad \sup _{\beta \in Q}\left|\mathcal{R}_{6, \beta}^{n}(1)\right| \leq \frac{C}{2 n^{1+1 / \alpha}}, \\
& \sup _{\beta \in Q}\left|\mathcal{R}_{7, \beta}^{n}(1)\right| \leq C \sup _{\beta \in Q}\left[\frac{\sup _{s \in[0,1]}\left|W_{s}^{n, \beta}\right|}{n^{1+1 / \alpha}\left(U_{1}^{n, \beta}\right)^{2}}\right], \quad \sup _{\beta \in Q}\left|\mathcal{R}_{8, \beta}^{n}(1)\right| \leq \frac{C}{n^{1+1 / \alpha}} .
\end{aligned}
$$


Moreover from (4.3)-(4.5), we get that

$$
\begin{aligned}
& \sup _{\beta \in Q} \frac{\sup _{s \in[0,1]}\left|L \bar{Y}_{s}^{n, \beta, x_{0}}\right|}{n^{1+1 / \alpha} U_{1}^{n, \beta}} \leq C\left[\frac{1}{n^{2+1 / \alpha}}+\frac{\int_{0}^{1} \int_{\mathbb{R}}\left[\left|\rho^{n^{\prime}}(z)\right|+\frac{1+\alpha}{|z|} \rho^{n}(z)\right] \mu^{(n)}(\mathrm{d} s, \mathrm{~d} z)}{n \int_{0}^{1} \int_{\mathbb{R}} \rho^{n}(z) \mu^{(n)}(\mathrm{d} s, \mathrm{~d} z)}\right] \\
&+C\left[\frac{\left.\int_{0}^{1} \int_{|z|>2} z^{2}\left|\frac{\tau^{\prime}\left(z / n^{1 / \alpha}\right)}{\tau\left(z / n^{1 / \alpha}\right)}\right| \mu^{(n)}(\mathrm{d} s, \mathrm{~d} z)\right]}{n^{1+1 / \alpha} \int_{0}^{1} \int_{|z|>2} z^{2} \mu^{(n)}(\mathrm{d} s, \mathrm{~d} z)}\right], \\
& \sup _{\beta \in Q} \frac{\sup _{s \in[0,1]}\left|W_{s}^{n, \beta}\right|}{n^{1+1 / \alpha}\left(U_{1}^{n, \beta}\right)^{2}} \leq C\left[\frac{1}{n^{2+1 / \alpha}}+\frac{\int_{0}^{1} \int_{\mathbb{R}} \rho^{n}(z)\left|\left(\rho^{n}\right)^{\prime}(z)\right| \mu^{(n)}(\mathrm{d} s, \mathrm{~d} z)}{n\left[\int_{0}^{1} \int_{\mathbb{R}} \rho^{n}(z) \mu^{(n)}(\mathrm{d} s, \mathrm{~d} z)\right]^{2}}\right] .
\end{aligned}
$$

From the results of Step 1, Step 2 in the proof of Theorem 2.2, and the control given in the proof of Theorem 3.2 for (4.11) we can deduce that $\sup _{\beta \in Q} \frac{\sup _{s \in[0,1]}\left|L \bar{Y}_{s}^{n, \beta, x_{0}}\right|}{n^{1+1 / \alpha} U_{1}^{n, \beta}}$ and $\sup _{\beta \in Q} \frac{\sup _{s \in[0,1]}\left|W_{s}^{n, \beta}\right|}{n^{1+1 / \alpha}\left(U_{1}^{n, \beta}\right)^{2}}$ converge to zero in $\mathbf{L}^{p}, \forall p \geq 1$ and we deduce the convergence of $\sup _{\beta \in Q}\left|\mathcal{R}_{5, \beta}^{n}(1)\right|$ and $\sup _{\beta \in Q}\left|\mathcal{R}_{7, \beta}^{n}(1)\right|$.

It remains to study the convergence of $\sup _{\beta \in Q}\left|\mathcal{R}_{4, \beta}^{n}(1)\right|$.

From the boundedness of $\left(\epsilon_{t}^{n}\right)_{t \in[0,1]}$, the definition of $\rho^{n}$ (see (3.11)), and since $\mu^{(n)}$ is a positive measure, we have

$$
\begin{aligned}
\sup _{\beta \in Q}\left|\mathcal{R}_{4, \beta}^{n}(1)\right| & \leq C\left[\frac{\int_{0}^{1} \int_{|z|>2}\left[\frac{|z|^{3}}{n^{1 / \alpha}}\left|\frac{\tau^{\prime}\left(z / n^{1 / \alpha}\right)}{\tau\left(z / n^{1 / \alpha}\right)}\right|+\frac{z^{4}}{n^{1 / \alpha}}\left(\left|\frac{\tau^{\prime \prime}\left(z / n^{1 / \alpha}\right)}{\tau\left(z / n^{1 / \alpha}\right)}\right|+\left(\frac{\tau^{\prime}\left(z / n^{1 / \alpha}\right)}{\tau\left(z / n^{1 / \alpha}\right)}\right)^{2}\right)\right] \mu^{(n)}(\mathrm{d} s, \mathrm{~d} z)}{\left(\int_{0}^{1} \int_{|z|>2} z^{2} \mu^{(n)}(\mathrm{d} s, \mathrm{~d} z)\right)^{2}}\right] \\
& \leq C\left[\frac{\int_{0}^{1} \int_{|z|>2}\left[\frac{|z|^{3}}{n^{1 / \alpha}}\left|\frac{\tau^{\prime}\left(z / n^{1 / \alpha}\right)}{\tau\left(z / n^{1 / \alpha}\right)}\right|+\frac{z^{4}}{n^{1 / \alpha}}\left(\left|\frac{\tau^{\prime \prime}\left(z / n^{1 / \alpha}\right)}{\tau\left(z / n^{1 / \alpha}\right)}\right|+\left(\frac{\tau^{\prime}\left(z / n^{1 / \alpha}\right)}{\tau\left(z / n^{1 / \alpha}\right)}\right)^{2}\right)\right] \mu^{(n)}(\mathrm{d} s, \mathrm{~d} z)}{n^{4 / \alpha}}\right],
\end{aligned}
$$

where we used $\int_{0}^{1} \int_{|z|>2} z^{2} \mu^{(n)}(\mathrm{d} s, \mathrm{~d} z)>n^{2 / \alpha}$, if there exists a jump of the Lévy process in $\left[-2 n^{1 / \alpha},-n^{1 / \alpha}\right) \cup$ $\left(n^{1 / \alpha}, 2 n^{1 / \alpha}\right]$. If there are no jumps in $\left[-2 n^{1 / \alpha},-n^{1 / \alpha}\right) \cup\left(n^{1 / \alpha}, 2 n^{1 / \alpha}\right]$, since $\tau\left(z / n^{1 / \alpha}\right)=1$ if $|z| \leq n^{1 / \alpha}$, we have $\tau^{\prime}\left(z / n^{1 / \alpha}\right)=0$ and $\tau^{\prime \prime}\left(z / n^{1 / \alpha}\right)=0$. Thus for $M(p)$ a positive constant we have

$$
\begin{aligned}
\mathbb{E} \sup _{\beta \in Q}\left|\mathcal{R}_{4, \beta}^{n}(1)\right|^{p} \leq & \frac{C^{p}}{n^{p / \alpha}} \mathbb{E}\left[\int _ { 0 } ^ { 1 } \int _ { | z | > 2 } \left[\frac{1}{n^{1 / \alpha}}\left(\left|\frac{z}{n^{1 / \alpha}}\right|\right)^{3}\left|\frac{\tau^{\prime}\left(z / n^{1 / \alpha}\right)}{\tau\left(z / n^{1 / \alpha}\right)}\right|\right.\right. \\
& \left.\left.+\left(\frac{z}{n^{1 / \alpha}}\right)^{4}\left(\left|\frac{\tau^{\prime \prime}\left(z / n^{1 / \alpha}\right)}{\tau\left(z / n^{1 / \alpha}\right)}\right|+\left(\frac{\tau^{\prime}\left(z / n^{1 / \alpha}\right)}{\tau\left(z / n^{1 / \alpha}\right)}\right)^{2}\right)\right] \mu^{(n)}(\mathrm{d} s, \mathrm{~d} z)\right]^{p}
\end{aligned}
$$




$$
\begin{aligned}
& \leq \frac{M(p)}{n^{p / \alpha}}\left[\mathbb{E}\left[\int_{0}^{1} \int_{|z|>2} \frac{1}{n^{1 / \alpha}}\left(\left|\frac{z}{n^{1 / \alpha}}\right|\right)^{3}\left|\frac{\tau^{\prime}\left(z / n^{1 / \alpha}\right)}{\tau\left(z / n^{1 / \alpha}\right)}\right| \mu^{(n)}(\mathrm{d} s, \mathrm{~d} z)\right]^{p}\right. \\
&+ \mathbb{E}\left[\int_{0}^{1} \int_{|z|>2}\left(\frac{z}{n^{1 / \alpha}}\right)^{4}\left|\frac{\tau^{\prime \prime}\left(z / n^{1 / \alpha}\right)}{\tau\left(z / n^{1 / \alpha}\right)}\right| \mu^{(n)}(\mathrm{d} s, \mathrm{~d} z)\right]^{p} \\
&\left.+\mathbb{E}\left[\int_{0}^{1} \int_{|z|>2}\left(\frac{z}{n^{1 / \alpha}}\right)^{4}\left(\frac{\tau^{\prime}\left(z / n^{1 / \alpha}\right)}{\tau\left(z / n^{1 / \alpha}\right)}\right)^{2} \mu^{(n)}(\mathrm{d} s, \mathrm{~d} z)\right]^{p}\right]
\end{aligned}
$$

Similarly to the proof of Theorem 3.2 , we show that under assumption $\mathbf{H}_{1}\left(b_{i i}\right), \sup _{\beta \in Q}\left|\mathcal{R}_{4, \beta}^{n}(1)\right|$ converges to zero as $n \rightarrow \infty$ in $\mathbf{L}^{p}$ for all $p \geq 1$ and this completes the proof of $\left.i i\right)$.

The result of $i i i)$ follows from Lemma $5.1 \mathrm{ii}$ ) and the estimation (5.45).

Proof of Lemma 5.3: i) From (5.5), the fact that $b$ has bounded derivatives, $\sup _{\beta} \sup _{0 \leq s \leq 1} \frac{U_{s}^{n, \beta}}{U_{1}^{n, \beta}}$ is bounded, the upper and lower bounds of $\left(\left(\epsilon_{t}^{n}\right)\right)_{t \in[0,1]}$, we deduce $\left.i\right)$.

ii) From (4.3), (5.9) we have

$$
\begin{aligned}
\frac{T_{1}^{n, \theta}}{n^{\frac{2}{\alpha}-1}\left(U_{1}^{n, \beta}\right)^{2}=} & \frac{3\left(\epsilon_{1}^{n}\right)^{3} \int_{0}^{1}\left(\epsilon_{1}^{n}\right)^{-3} b^{\prime \prime}\left(\bar{Y}_{s}^{n, \beta, x_{0}}, \theta\right) V_{s}^{n, \theta} U_{s}^{n, \beta} \mathrm{d} s}{n^{\frac{2}{\alpha}}\left(U_{1}^{n, \beta}\right)^{2}}+\frac{\left(\epsilon_{1}^{n}\right)^{3} \int_{0}^{1}\left(\epsilon_{1}^{n}\right)^{-3}\left(\partial_{\theta} b\right)^{\prime}\left(\bar{Y}_{s}^{n, \beta, x_{0}}, \theta\right) W_{s}^{n, \beta} \mathrm{d} s}{n^{\frac{2}{\alpha}}\left(U_{1}^{n, \beta}\right)^{2}} \\
& +\frac{\left(\epsilon_{1}^{n}\right)^{3} \int_{0}^{1}\left(\epsilon_{1}^{n}\right)^{-3} b^{\prime \prime}\left(\bar{Y}_{s}^{n, \beta, x_{0}}, \theta\right) \partial_{\theta} \bar{Y}_{s}^{n, \beta, x_{0}} W_{s}^{n, \beta} \mathrm{d} s}{n^{\frac{2}{\alpha}}\left(U_{1}^{n, \beta}\right)^{2}}+\frac{\left(\epsilon_{1}^{n}\right)^{3} \int_{0}^{1}\left(\epsilon_{1}^{n}\right)^{-3}\left(\partial_{\theta} b\right)^{\prime \prime}\left(\bar{Y}_{s}^{n, \beta, x_{0}}, \theta\right)\left(U_{s}^{n, \beta}\right)^{2} \mathrm{~d} s}{n^{\frac{2}{\alpha}}\left(U_{1}^{n, \beta}\right)^{2}} \\
& +\frac{\left(\epsilon_{1}^{n}\right)^{3} \int_{0}^{1}\left(\epsilon_{1}^{n}\right)^{-3} b^{\prime \prime \prime}\left(\bar{Y}_{s}^{n, \beta, x_{0}}, \theta\right) \partial_{\theta} \bar{Y}_{s}^{n, \beta, x_{0}}\left(U_{s}^{n, \beta}\right)^{2} \mathrm{~d} s}{n^{\frac{2}{\alpha}}\left(U_{1}^{n, \beta}\right)^{2}}
\end{aligned}
$$

We deduce, using Lemma $5.1 i$ ) and Lemma $5.3 i$ ) that

$$
\sup _{\beta \in Q}\left|\frac{T_{1}^{n, \theta}}{n^{\frac{2}{\alpha}-1}\left(U_{1}^{n, \beta}\right)^{2}}\right| \leq \frac{C_{1}}{n^{\frac{2}{\alpha}}}+C_{2} \sup _{\beta \in Q} \frac{\sup _{s \in(0,1]}\left|W_{s}^{n, \beta}\right|}{n^{\frac{2}{\alpha}}\left(U_{1}^{n, \beta}\right)^{2}}
$$

where $C_{1}, C_{2}$ are some deterministic constants. Now from the estimation (5.45), we easily deduce that $\sup _{\beta \in Q} \frac{\sup _{s \in(0,1]}\left|W_{s}^{n, \beta}\right|}{n^{\frac{2}{\alpha}}\left(U_{1}^{n, \beta}\right)^{2}}$ tends to zero as $n \rightarrow \infty$ and then we get $\left.i i\right)$.

$* * * i i i)$ and $i v)$ From $(4.3),(5.6),(5.10)$, an easy computation shows the decomposition of $\frac{V_{1}^{n, \sigma}}{U_{1}^{n, \beta}}$ and $\frac{1}{n^{1 / \alpha}} \frac{T_{1}^{n, \sigma}}{\left(U_{1}^{n, \beta}\right)^{2}}$, where the leading term is (5.18) and the remainder terms are given by

$$
\mathcal{R}_{9, \beta}^{n}(1)=\frac{\left(\epsilon_{1}^{n}\right)^{2} \int_{0}^{1}\left(\epsilon_{s}^{n}\right)^{-2}\left(b^{\prime \prime}\left(\bar{Y}_{s}^{n, \beta, x_{0}}, \theta\right) \partial_{\sigma} \bar{Y}_{s}^{n, \beta, x_{0}} U_{s}^{n, \beta}\right) \mathrm{d} s}{n U_{1}^{n, \beta}},
$$




$$
\begin{aligned}
& \mathcal{R}_{10, \beta}^{n}(1)=\frac{3\left(\epsilon_{1}^{n}\right)^{3} \int_{0}^{1}\left(\epsilon_{1}^{n}\right)^{-3} b^{\prime \prime}\left(\bar{Y}_{s}^{n, \beta, x_{0}}, \theta\right) V_{s}^{n, \sigma} U_{s}^{n, \beta} \mathrm{d} s}{n^{1+1 / \alpha}\left(U_{1}^{n, \beta}\right)^{2}}, \\
& \mathcal{R}_{11, \beta}^{n}(1)=\frac{\left(\epsilon_{1}^{n}\right)^{3} \int_{0}^{1}\left(\epsilon_{1}^{n}\right)^{-3} b^{\prime \prime}\left(\bar{Y}_{s}^{n, \beta, x_{0}}, \theta\right) \partial_{\sigma} \bar{Y}_{s}^{n, \beta, x_{0}} W_{s}^{n, \beta} \mathrm{d} s}{n^{1+1 / \alpha}\left(U_{1}^{n, \beta}\right)^{2}}, \\
& \mathcal{R}_{12, \beta}^{n}(1)=\frac{\left(\epsilon_{1}^{n}\right)^{3} \int_{0}^{1}\left(\epsilon_{1}^{n}\right)^{-3} b^{\prime \prime \prime}\left(\bar{Y}_{s}^{n, \beta, x_{0}}, \theta\right) \partial_{\sigma} \bar{Y}_{s}^{n, \beta, x_{0}}\left(U_{s}^{n, \beta}\right)^{2} \mathrm{~d} s}{n^{1+1 / \alpha}\left(U_{1}^{n, \beta}\right)^{2}} .
\end{aligned}
$$

Moreover, using that $b$ has bounded derivatives and $\sup _{\beta} \sup _{0 \leq s \leq 1} \frac{U_{s}^{n, \beta}}{U_{1}^{n, \beta}}$ is bounded, the remainder terms satisfy the upper bounds

$$
\begin{aligned}
& \sup _{\beta \in Q}\left|\mathcal{R}_{9, \beta}^{n}(1)\right| \leq \frac{C}{n} \sup _{\beta \in Q} \sup _{s \in[0,1]}\left|\partial_{\sigma} \bar{Y}_{s}^{n, \beta, x_{0}}\right|, \quad \sup _{\beta \in Q}\left|\mathcal{R}_{10, \beta}^{n}(1)\right| \leq \frac{C_{1}}{n^{1+1 / \alpha}} \sup _{\beta \in Q}\left[\frac{\sup _{s \in[0,1]}\left|V_{s}^{n, \sigma}\right|}{U_{1}^{n, \beta}}\right], \\
& \sup _{\beta \in Q}\left|\mathcal{R}_{11, \beta}^{n}(1)\right| \leq \frac{C_{2}}{n^{1+1 / \alpha}} \sup _{\beta \in Q}\left[\frac{\sup _{s \in[0,1]}\left|\partial_{\sigma} \bar{Y}_{s}^{n, \beta, x_{0}} W_{s}^{n, \beta}\right|}{\left(U_{1}^{n, \beta}\right)^{2}}\right], \quad \sup _{\beta \in Q}\left|\mathcal{R}_{12, \beta}^{n}(1)\right| \leq \frac{C_{3}}{n^{1+1 / \alpha}} \sup _{\beta \in Q} \sup _{s \in[0,1]}\left|\partial_{\sigma} \bar{Y}_{s}^{n, \beta, x_{0}}\right|,
\end{aligned}
$$

where $C, C_{1}, C_{2}, C_{3}$ are deterministic constants.

We observe that from Lemmas 5.1 and 5.2 iii), we can deduce immediately the convergences to zero in $\mathbf{L}^{p}, \forall p \geq 1$ of the remainder terms $\sup _{\beta \in Q}\left|\mathcal{R}_{9, \beta}^{n}(1)\right|, \sup _{\beta \in Q}\left|\mathcal{R}_{11, \beta}^{n}(1)\right|$ and $\sup _{\beta \in Q}\left|\mathcal{R}_{12, \beta}^{n}(1)\right|$.

For $\sup _{\beta \in Q}\left|\mathcal{R}_{10, \beta}^{n}(1)\right|$, the proof follows from Lemma 5.1 ii), (5.6), (4.3), the boundedness of $\left(\epsilon_{s}^{n}\right)$, the fact that $b$ has bounded derivatives and $\sup _{\beta} \sup _{s} \frac{U_{s}^{n, \beta}}{U_{1}^{n, \beta}}$ is bounded.

The convergence of $\widehat{\mathcal{H}}_{5, \beta_{n}}^{n}(1)$ is proved as the convergence of $\widehat{\mathcal{H}}_{3, \beta_{n}}^{n}(1)$ in the proof of Lemma 3.3. This completes the proof Lemma 5.3.

Proof of Lemma 5.4: We first prove (5.20). From (5.3) we have (we omit the details)

$$
\sup _{x_{0}}\left|n \partial_{\theta} \bar{Y}_{1}^{n, \beta_{n}, x_{0}}-\partial_{\theta} b\left(x_{0}, \theta\right)\right| \underset{a . s}{\stackrel{n \rightarrow \infty}{\longrightarrow}} 0 .
$$

From the expressions (3.14), (3.15), using $\sup _{x_{0}} \sup _{s \in[0,1]}\left|\epsilon_{s}^{n}-1\right| \rightarrow 0$ and Lemma 3.1, it can be seen that

$$
\sup _{x_{0}}\left|\widehat{\mathcal{H}}_{\beta_{n}}^{n}(1)^{q}-\mathcal{H}_{L^{\alpha}}(1)^{q}\right| \underset{a . s}{\stackrel{n \rightarrow \infty}{\longrightarrow}} 0, \quad \forall q \geq 1 .
$$

We deduce that almost surely, one has the convergence

$$
\forall q \geq 1, \quad \sup _{x_{0}}\left|n \partial_{\theta} \bar{Y}_{1}^{n, \beta_{n}, x_{0}}\left(\widehat{\mathcal{H}}_{\beta_{n}}^{n}(1)\right)^{q}-\partial_{\theta} b\left(x_{0}, \theta\right)\left(\mathcal{H}_{L^{\alpha}}(1)\right)^{q}\right| \stackrel{n \rightarrow \infty}{\longrightarrow} 0 .
$$

Using (4.24) and $\sup _{\beta, x_{0}}\left|\partial_{\theta} \bar{Y}_{1}^{n, \beta, x_{0}}\right| \leq \frac{C}{n}$, we can apply the dominated convergence theorem and see that the convergence (5.50) holds in $\mathbf{L}^{p}$-norm for all $p \geq 1$.

For (5.21): the proof is similar to (5.20). 
For (5.22): using (5.4), Gronwall's Lemma and Lemma 3.1, we can prove

$$
\sup _{x_{0}}\left|n^{1 / \alpha} \partial_{\sigma} \bar{Y}_{1}^{n, \beta_{n}, x_{0}}-L_{1}^{\alpha}\right| \stackrel{n \rightarrow \infty}{a . s} 0
$$

and from (5.49) we deduce

$$
\forall q \geq 1, \quad \sup _{x_{0}}\left|n^{1 / \alpha} \partial_{\sigma} \bar{Y}_{1}^{n, \beta_{n}, x_{0}}\left(\widehat{\mathcal{H}}_{\beta_{n}}^{n}(1)\right)^{q}-L_{1}^{\alpha}\left(\mathcal{H}_{L^{\alpha}}(1)\right)^{q}\right| \stackrel{n \rightarrow \infty}{\longrightarrow} 0 .
$$

Consequently, to prove the convergence in $\mathbf{L}^{p}$-norm, it remains to check

$$
\forall p, q \geq 1, \quad \mathbb{E} \sup _{n, \beta, x_{0}}\left|n^{1 / \alpha} \partial_{\sigma} \bar{Y}_{1}^{n, \beta_{n}, x_{0}}\left(\widehat{\mathcal{H}}_{\beta}^{n}(1)\right)^{q}\right|^{p}<\infty
$$

Using again (5.4) and Gronwall's Lemma, we have

$$
\sup _{x_{0}, \beta}\left|n^{1 / \alpha} \partial_{\sigma} \bar{Y}_{1}^{n, \beta, x_{0}}\right| \leq C \sup _{t \in[0,1]}\left|L_{t}^{n}\right|
$$

and (5.51) reduces to

$$
\forall p, q \geq 1, \quad \mathbb{E} \sup _{n, \beta, x_{0}}\left|\sup _{t \in[0,1]}\right| L_{t}^{n}\left|\left(\widehat{\mathcal{H}}_{\beta}^{n}(1)\right)^{q}\right|^{p}<\infty
$$

Let us recall that $L_{t}^{n}=\int_{0}^{t} \int_{|z| \leq 1} z \tilde{\mu}(\mathrm{d} s, \mathrm{~d} z)+\int_{0}^{t} \int_{|z|>1} z \mu^{(n)}(\mathrm{d} s, \mathrm{~d} z)$. Then we have

$$
\sup _{t \in[0,1]}\left|L_{t}^{n}\right| \leq \sup _{t \in[0,1]}\left|\int_{0}^{t} \int_{|z| \leq 1} z \tilde{\mu}(\mathrm{d} s, \mathrm{~d} z)\right|+\int_{0}^{1} \int_{|z|>1}|z| \mu^{(n)}(\mathrm{d} s, \mathrm{~d} z) .
$$

From this decomposition and since $\sup _{t \in[0,1]}\left|\int_{0}^{t} \int_{|z| \leq 1} z \tilde{\mu}(\mathrm{d} s, \mathrm{~d} z)\right|^{p}$ is integrable, for all $p \geq 1$, we see that (5.52) is a consequence of (4.24) and the following bound

$$
\forall p \geq 1, \quad \mathbb{E} \sup _{n, \beta, x_{0}}\left|\widehat{\mathcal{H}}_{\beta}^{n}(1) \int_{0}^{1} \int_{|z|>1}\right| z\left|\mu^{(n)}(\mathrm{d} s, \mathrm{~d} z)\right|^{p}<\infty .
$$

To prove (5.53), we first remark from (4.17) that for $C$ a positive constant,

$$
\begin{aligned}
\left|\widehat{\mathcal{H}}_{\beta}^{n}(1) \int_{0}^{1} \int_{|z|>1}\right| z\left|\mu^{(n)}(\mathrm{d} s, \mathrm{~d} z)\right| \leq & C\left[\left(\frac{\int_{0}^{1} \int_{\mathbb{R}} \rho^{n}(z)\left|\left(\rho^{n}\right)^{\prime}(z)\right| \mu^{(n)}(\mathrm{d} s, \mathrm{~d} z)}{\left[\int_{0}^{1} \int_{\mathbb{R}} \rho^{n}(z) \mu^{(n)}(\mathrm{d} s, \mathrm{~d} z)\right]^{2}}+\frac{\int_{0}^{1} \int_{\mathbb{R}}\left[\left|\rho^{n^{\prime}}(z)\right|+\frac{1+\alpha}{|z|} \rho^{n}(z)\right] \mu^{(n)}(\mathrm{d} s, \mathrm{~d} z)}{\int_{0}^{1} \int_{\mathbb{R}} \rho^{n}(z) \mu^{(n)}(\mathrm{d} s, \mathrm{~d} z)}\right)\right. \\
& \left.\times \int_{0}^{1} \int_{2 \geq|z|>1}|z| \mu(\mathrm{d} s, \mathrm{~d} z)\right]+C\left[\left(\frac{\int_{0}^{1} \int_{\mathbb{R}} \rho^{n}(z)\left|\left(\rho^{n}\right)^{\prime}(z)\right| \mu^{(n)}(\mathrm{d} s, \mathrm{~d} z)}{\left[\int_{0}^{1} \int_{\mathbb{R}} \rho^{n}(z) \mu^{(n)}(\mathrm{d} s, \mathrm{~d} z)\right]^{2}}\right.\right. \\
& \left.+\frac{\int_{0}^{1} \int_{\mathbb{R}}\left[\left|\rho^{n}(z)\right|+\frac{1+\alpha}{|z|} \rho^{n}(z)\right] \mu^{(n)}(\mathrm{d} s, \mathrm{~d} z)}{\int_{0}^{1} \int_{\mathbb{R}} \rho^{n}(z) \mu^{(n)}(\mathrm{d} s, \mathrm{~d} z)} \int_{0}^{1} \int_{|z|>2}|z| \mu^{(n)}(\mathrm{d} s, \mathrm{~d} z)\right]
\end{aligned}
$$


Considering the first term in the right-hand side of (5.54), from the proofs of Step $\mathbf{1}$ and Step $\mathbf{2}$ in Theorem 2.2, we deduce that it is bounded by a random variable independent of $n, \beta$ and $x_{0}$ and belonging to $\cap_{p \geq 1} \mathbf{L}^{p}$.

We now consider the second term in the right-hand side of (5.54). From (3.11), we have

$$
\begin{aligned}
& \left|\left(\frac{\int_{0}^{1} \int_{\mathbb{R}} \rho^{n}(z)\left|\left(\rho^{n}\right)^{\prime}(z)\right| \mu^{(n)}(\mathrm{d} s, \mathrm{~d} z)}{\left[\int_{0}^{1} \int_{\mathbb{R}} \rho^{n}(z) \mu^{(n)}(\mathrm{d} s, \mathrm{~d} z)\right]^{2}}+\frac{\int_{0}^{1} \int_{\mathbb{R}}\left[\left|\rho^{n^{\prime}}(z)\right|+\frac{1+\alpha}{|z|} \rho^{n}(z)\right] \mu^{(n)}(\mathrm{d} s, \mathrm{~d} z)}{\int_{0}^{1} \int_{\mathbb{R}} \rho^{n}(z) \mu^{(n)}(\mathrm{d} s, \mathrm{~d} z)}\right) \int_{0}^{1} \int_{|z|>2}\right| z\left|\mu^{(n)}(\mathrm{d} s, \mathrm{~d} z)\right| \\
& \leq\left[\frac{\int_{0}^{1} \int_{|z| \leq 2} \rho(z)\left|\rho^{\prime}(z)\right| \mu(\mathrm{d} s, \mathrm{~d} z)}{\left(\int_{0}^{1} \int_{|z|>2} z^{2} \mu^{(n)}(\mathrm{d} s, \mathrm{~d} z)\right)^{2}}+\frac{\int_{0}^{1} \int_{|z| \leq 2}\left(\left|\rho^{\prime}(z)\right|+\rho(z) \frac{1+\alpha}{|z|}\right) \mu(\mathrm{d} s, \mathrm{~d} z)}{\int_{0}^{1} \int_{|z|>2} z^{2} \mu^{(n)}(\mathrm{d} s, \mathrm{~d} z)}\right] \int_{0}^{1} \int_{|z|>2}|z| \mu^{(n)}(\mathrm{d} t, \mathrm{~d} z) \\
& \quad+\left[\frac{\int_{0}^{1} \int_{|z|>2} 2|z|^{3} \mu^{(n)}(\mathrm{d} s, \mathrm{~d} z)}{\left(\int_{0}^{1} \int_{|z|>2} z^{2} \mu^{(n)}(\mathrm{d} s, \mathrm{~d} z)\right)^{2}}+\frac{\int_{0}^{1} \int_{|z|>2}(3+\alpha)|z| \mu^{(n)}(\mathrm{d} s, \mathrm{~d} z)}{\int_{0}^{1} \int_{|z|>2} z^{2} \mu^{(n)}(\mathrm{d} s, \mathrm{~d} z)}\right] \int_{0}^{1} \int_{|z|>2}|z| \mu^{(n)}(\mathrm{d} t, \mathrm{~d} z)
\end{aligned}
$$

Using the Cauchy-Schwarz inequality $\int_{0}^{1} \int_{|z|>2} \mu^{n}(\mathrm{~d} t, \mathrm{~d} z) \times \int_{0}^{1} \int_{|z|>2} z^{2} \mu^{(n)}(\mathrm{d} s, \mathrm{~d} z) \geq\left(\int_{0}^{1} \int_{|z|>2}|z| \mu^{n}(\mathrm{~d} t, \mathrm{~d} z)\right)^{2}$ we get:

$$
\begin{aligned}
\frac{\int_{0}^{1} \int_{|z|>2} 2|z|^{3} \mu^{(n)}(\mathrm{d} s, \mathrm{~d} z)}{\left(\int_{0}^{1} \int_{|z|>2} z^{2} \mu^{(n)}(\mathrm{d} s, \mathrm{~d} z)\right)^{2}} \int_{0}^{1} \int_{|z|>2}|z| \mu^{(n)}(\mathrm{d} t, \mathrm{~d} z) & \leq \frac{\int_{0}^{1} \int_{|z|>2} 2|z|^{3} \mu^{(n)}(\mathrm{d} s, \mathrm{~d} z)}{\left(\int_{0}^{1} \int_{|z|>2}|z| \mu^{(n)}(\mathrm{d} t, \mathrm{~d} z)\right)^{3}}\left(\int_{0}^{1} \int_{|z|>2} \mu^{(n)}(\mathrm{d} t, \mathrm{~d} z)\right)^{2} \\
& =\frac{\sum_{i=1}^{N_{1}} 2\left|Z_{i}\right|^{3}}{\left(\sum_{i=1}^{N_{1}}\left|Z_{i}\right|\right)^{3}}\left(\int_{0}^{1} \int_{|z|>2} \mu^{(n)}(\mathrm{d} t, \mathrm{~d} z)\right)^{2} \\
& \leq 2\left(\int_{0}^{1} \int_{|z|>2} \mu(\mathrm{d} t, \mathrm{~d} z)\right)^{2}
\end{aligned}
$$

and

$$
\frac{\int_{0}^{1} \int_{|z|>2}(3+\alpha)|z| \mu^{(n)}(\mathrm{d} s, \mathrm{~d} z)}{\int_{0}^{1} \int_{|z|>2} z^{2} \mu^{(n)}(\mathrm{d} s, \mathrm{~d} z)} \int_{0}^{1} \int_{|z|>2}|z| \mu^{n}(\mathrm{~d} t, \mathrm{~d} z) \leq(3+\alpha) \int_{0}^{1} \int_{|z|>2} \mu(\mathrm{d} t, \mathrm{~d} z) .
$$

Combining (5.56), (5.57) with (5.55), it follows that the second term in the right-hand side of (5.54) is also bounded by a random variable independent of $n, \beta$ and $x_{0}$ and belonging to $\cap_{p \geq 1} \mathbf{L}^{p}$. Consequently, we get (5.53) and this achieves the proof of (5.22).

For (5.23), the proof is similar to (5.22).

Proof of Lemma 5.5: From (5.13), we prove $i$ ) and $i i$ ) proceeding as in the proof of (5.20), and iii) and $i v$ ) proceeding as in the proof of $(5.22)$.

\section{Appendix A Representation of the transition DENSITy Via MALLIAVIN CALCULUS}

The aim of this section is to represent the density of a pure jump Lévy process as well as its derivative and its logarithm derivative as an expectation, using the Malliavin calculus for jump processes developed by Bichteler 
et al. [2] and used by Clément and Gloter [5]. We are not exactly in the context of [2], where the compensator of the Poisson measure is uniform on $[0,1] \times E$. In fact, in our context the compensator of the Poisson measure is $\mathrm{d} t \times g(z) \mathrm{d} z$, where $g$ is the density of the Lévy measure and we need to adapt slightly the definitions of Malliavin operators given in [2]. We recall here the appropriate integration by parts setting developed in [5] for the reader convenience.

\section{A.1 Integration by parts setting}

We consider a filtered probability space $\left(\Omega, \mathcal{G},\left(\mathcal{G}_{t}\right)_{t \in[0,1]}, \mathbb{P}\right)$ endowed with a Poisson random measure $\mu$ on $[0,1] \times E$, where $E$ is an open subset of $\mathbb{R}$, with compensator $v$ on $[0,1] \times E$ and with compensated measure $\tilde{\mu}=\mu-v$. We now consider the process $\left(Y_{t}^{\beta}\right)_{t \in[0,1]}$, the solution of

$$
Y_{t}^{\beta}=y_{0}+\int_{0}^{t} a\left(Y_{s}^{\beta}, \theta\right) \mathrm{d} s+c \sigma \int_{0}^{t} \int_{E} z \tilde{\mu}(\mathrm{d} s, \mathrm{~d} z)
$$

where the parameter $\beta=(\theta, \sigma)^{T}$ belongs to $\mathbb{R} \times(0, \infty), a$ is a real valued function and $c$ is a constant.

This is the framework of Clément and Gloter [5] and our aim is to give some explicit representation formulas for the density of $Y_{1}^{\beta}$ and its derivative with respect to $\beta$.

We assume that the following assumptions are fulfilled.

H: (a) The function $a$ has bounded derivatives up to order five with respect to both variables.

(b) The compensator of the Poisson random measure $\mu$ is given by $v(\mathrm{~d} t, \mathrm{~d} z)=\mathrm{d} t \times g(z) \mathrm{d} z$ with $g \geq 0$ on $E, \mathcal{C}^{1}$ on $E$ and such that

$$
\forall p \geq 2, \int_{E}|z|^{p} g(z) \mathrm{d} z<\infty
$$

We now recall the Malliavin operators $L$ and $\Gamma$ and their basic properties (see Bichteler et al. [2], Chapter IV, Section 8-9-10). For a test function $f:[0,1] \times E \mapsto \mathbb{R}\left(f\right.$ is measurable, $\mathcal{C}^{2}$ with respect to the second variable, with bounded derivative, and $\left.f \in \cap_{p \geq 1} \mathbf{L}^{p}(\nu)\right)$ we set $\mu(f)=\int_{0}^{1} \int_{E} f(t, z) \mu(\mathrm{d} t, \mathrm{~d} z)$. We introduce an auxiliary function $\rho: E \mapsto(0, \infty)$ such that $\rho$ admits a derivative and $\rho, \rho^{\prime}$ and $\rho \frac{g^{\prime}}{g}$ belong to $\cap_{p \geq 1} \mathbf{L}^{p}(g(z) \mathrm{d} z)$. With these notations, we define the Malliavin operator $L$, on a simple functional $\mu(f)$, in the same way as in [5] by the following equations :

$$
L(\mu(f))=\frac{1}{2} \mu\left(\rho^{\prime} f^{\prime}+\rho \frac{g^{\prime}}{g} f^{\prime}+\rho f^{\prime \prime}\right)
$$

where $f^{\prime}$ and $f^{\prime \prime}$ are the derivatives with respect to the second variable. For $\Phi=F\left(\mu\left(f_{1}\right), . ., \mu\left(f_{k}\right)\right)$, with $F$ of class $\mathcal{C}^{2}$, we set

$$
L \Phi=\sum_{i=1}^{k} \frac{\partial F}{\partial x_{i}}\left(\mu\left(f_{1}\right), \ldots, \mu\left(f_{k}\right)\right) L\left(\mu\left(f_{i}\right)\right)+\frac{1}{2} \sum_{i, j=1}^{k} \frac{\partial^{2} F}{\partial x_{i} \partial x_{j}}\left(\mu\left(f_{1}\right), \ldots, \mu\left(f_{k}\right)\right) \mu\left(\rho f_{i}^{\prime} f_{j}^{\prime}\right)
$$

These definitions permit to construct a linear operator $L$ on a space $D \subset \cap_{p \geq 1} \mathbf{L}^{p}$ with the same basic properties as in equations. (i)-(iii), p.2322 from [5].

We associate to $L$, the symmetric bilinear operator $\Gamma$ :

$$
\Gamma(\Phi, \Psi)=L(\Phi \Psi)-\Phi L \Psi-\Psi L \Phi .
$$


Moreover, if $f$ and $h$ are two test functions, we have:

$$
\Gamma(\mu(f), \mu(h))=\mu\left(\rho f^{\prime} h^{\prime}\right) .
$$

These operators satisfy the following properties (see [2], Eq. (8-3))

$$
\begin{aligned}
L F(\Phi) & =F^{\prime}(\Phi) L \Phi+\frac{1}{2} F^{\prime \prime}(\Phi) \Gamma(\Phi, \Phi), \\
\Gamma(F(\Phi), \Psi) & =F^{\prime}(\Phi) \Gamma(\Phi, \Psi), \\
\Gamma\left(F\left(\Phi_{1}, \Phi_{2}\right), \Psi\right) & =\partial_{\Phi_{1}} F\left(\Phi_{1}, \Phi_{2}\right) \Gamma\left(\Phi_{1}, \Psi\right)+\partial_{\Phi_{2}} F\left(\Phi_{1}, \Phi_{2}\right) \Gamma\left(\Phi_{2}, \Psi\right) .
\end{aligned}
$$

The operator $L$ and the operator $\Gamma$ permit to establish the following integration by parts formula (see [2], Props. 8-10, p.103).

Proposition A.1. For $\Phi$ and $\Psi$ in $D$, and $f$ bounded with bounded derivatives up to order two, we have

$$
\mathbb{E} f^{\prime}(\Phi) \Psi \Gamma(\Phi, \Phi)=\mathbb{E} f(\Phi)(-2 \Psi L \Phi-\Gamma(\Phi, \Psi)) .
$$

Morover, if $\Gamma(\Phi, \Phi)$ is invertible and $\Gamma^{-1}(\Phi, \Phi) \in \cap_{p \geq 1} \boldsymbol{L}^{p}$, we have

$$
\mathbb{E} f^{\prime}(\Phi) \Psi=\mathbb{E} f(\Phi) \mathcal{H}_{\Phi}(\Psi),
$$

with

$$
\begin{aligned}
\mathcal{H}_{\Phi}(\Psi) & =-2 \Psi \Gamma^{-1}(\Phi, \Phi) L \Phi-\Gamma\left(\Phi, \Psi \Gamma^{-1}(\Phi, \Phi)\right) \\
& =-2 \Psi \Gamma^{-1}(\Phi, \Phi) L \Phi-\frac{1}{\Gamma(\Phi, \Phi)} \Gamma(\Phi, \Psi)+\frac{\Psi}{\Gamma(\Phi, \Phi)^{2}} \Gamma(\Phi, \Gamma(\Phi, \Phi)) .
\end{aligned}
$$

\section{A.2 Representation of the density of $Y_{1}^{\beta}$ and its derivative}

The integration by parts setting of the preceding section permits to derive the existence of the density of $Y_{1}^{\beta}$ given by (A.1), and gives a representation of this density as an expectation. From Bichteler et al. ([2], Sect. 10, p.130) we know that $\forall t>0$, the variable $Y_{t}^{\beta}$, the solution of (A.1), belongs to the domain of the operator $L$, and we can compute $L Y_{t}^{\beta}$ and $\Gamma\left(Y_{t}^{\beta}, Y_{t}^{\beta}\right)$ as in [5]. We recall the representation formula for the density of $Y_{1}^{\beta}$ (see [5]).

Theorem A.2. [Clément and Gloter [5]]: Let us denote by $q^{\beta}$ the density of $Y_{1}^{\beta}$. We assume that $\boldsymbol{H}$ holds and that the auxiliary function $\rho$ satisfies:

$$
\liminf _{u \rightarrow \infty} \frac{1}{\ln u} \int_{E} 1_{\{\rho(z) \geq 1 / u\}} g(z) \mathrm{d} z=+\infty
$$

Then,

$$
q^{\beta}(u)=\mathbb{E}\left(1_{\left\{Y_{1}^{\beta} \geq u\right\}} \mathcal{H}_{Y_{1}^{\beta}}(1)\right)
$$

with,

$$
\mathcal{H}_{Y_{1}^{\beta}}(1)=\frac{\Gamma\left(Y_{1}^{\beta}, \Gamma\left(Y_{1}^{\beta}, Y_{1}^{\beta}\right)\right)}{\Gamma\left(Y_{1}^{\beta}, Y_{1}^{\beta}\right)^{2}}-2 \frac{L Y_{1}^{\beta}}{\Gamma\left(Y_{1}^{\beta}, Y_{1}^{\beta}\right)}=\frac{W_{1}^{\beta}}{\left(U_{1}^{\beta}\right)^{2}}-2 \frac{L Y_{1}^{\beta}}{U_{1}^{\beta}},
$$


where the processes $\left(L Y_{t}^{\beta}\right)$ and $\left(U_{t}^{\beta}\right)=\Gamma\left(Y_{t}^{\beta}, Y_{t}^{\beta}\right)$ are solutions of the linear equations:

$$
\begin{aligned}
L Y_{t}^{\beta} & =\int_{0}^{t} a^{\prime}\left(Y_{s}^{\beta}, \theta\right) L Y_{s}^{\beta} \mathrm{d} s+\frac{1}{2} \int_{0}^{t} a^{\prime \prime}\left(Y_{s}^{\beta}, \theta\right) U_{s}^{\beta} \mathrm{d} s+\frac{c \sigma}{2} \int_{0}^{t} \int_{E}\left(\rho^{\prime}(z)+\rho(z) \frac{g^{\prime}(z)}{g(z)}\right) \mu(\mathrm{d} s, \mathrm{~d} z) \\
U_{t}^{\beta} & =2 \int_{0}^{t} a^{\prime}\left(Y_{s}^{\beta}, \theta\right) U_{s}^{\beta} \mathrm{d} s+c^{2} \sigma^{2} \int_{0}^{1} \int_{E} \rho(z) \mu(\mathrm{d} s, \mathrm{~d} z) .
\end{aligned}
$$

The process $\left(W_{t}^{\beta}\right)=\Gamma\left(Y_{t}^{\beta}, U_{t}^{\beta}\right)$ is the solution of the linear equation:

$$
W_{t}^{\beta}=3 \int_{0}^{t} a^{\prime}\left(Y_{s}^{\beta}, \theta\right) W_{s}^{\beta} \mathrm{d} s+2 \int_{0}^{t} a^{\prime \prime}\left(Y_{s}^{\beta}, \theta\right)\left(U_{s}^{\beta}\right)^{2} \mathrm{~d} s+c^{3} \sigma^{3} \int_{0}^{t} \int_{E} \rho(z) \rho^{\prime}(z) \mu(\mathrm{d} s, \mathrm{~d} z)
$$

In [5], the authors studied the derivative of $q^{\beta}$ with respect to the drift parameter $\theta$ only. Here, we intend to study the derivative of $q^{\beta}$ with respect to both parameters $\theta$ and $\sigma$. We first remark that $\left(Y_{t}^{\beta}\right)_{t}$ admits derivatives with respect to $\theta$ and $\sigma$ (see [2], Thm. 5.24 p.51), denoted by $\left(\partial_{\theta} Y_{t}^{\beta}\right)_{t}$ and $\left(\partial_{\sigma} Y_{t}^{\beta}\right)_{t}$ respectively. Moreover, $\left(\partial_{\theta} Y_{t}^{\beta}\right)_{t},\left(\partial_{\sigma} Y_{t}^{\beta}\right)_{t}$ are respectively the unique solutions of

$$
\begin{gathered}
\partial_{\theta} Y_{t}^{\beta}=\int_{0}^{t} a^{\prime}\left(Y_{s}^{\beta}, \theta\right) \partial_{\theta} Y_{s}^{\beta} \mathrm{d} s+\int_{0}^{t} \partial_{\theta} a\left(Y_{s}^{\beta}, \theta\right) \mathrm{d} s, \\
\partial_{\sigma} Y_{t}^{\beta}=\int_{0}^{t} a^{\prime}\left(Y_{s}^{\beta}, \theta\right) \partial_{\sigma} Y_{s}^{\beta} \mathrm{d} s+c \int_{0}^{t} \int_{E} z \tilde{\mu}(\mathrm{d} s, \mathrm{~d} z) .
\end{gathered}
$$

By iterating the integration by parts formula, since $Y_{1}^{\beta}$ admits derivatives with respect to $\theta$ and $\sigma$, one can prove, under the assumption $\mathbf{H}$, the existence and the continuity in $\beta$ of $\nabla_{\beta} q^{\beta}$ (see Thm. 4-21 in [2]), moreover, we will represent it as an expectation in Theorem A.4. The next result extends the result of Theorem 5 in [5], by giving an expression for the logarithm derivatives of the density w.r.t. $(\theta, \sigma)$ in terms of a conditional expectation.

Theorem A.3. Under the assumptions of Theorem A.2,

$$
\frac{\nabla_{\beta} q^{\beta}}{q^{\beta}}(u)=\left(\begin{array}{c}
\frac{\partial_{\theta} q^{\beta}}{q^{\beta}}(u) \\
\frac{\partial_{\sigma} q^{\beta}}{q^{\beta}}(u)
\end{array}\right)=\mathbb{E}\left(\mathcal{H}_{Y_{1}^{\beta}}\left(\nabla_{\beta} Y_{1}^{\beta}\right) \mid Y_{1}^{\beta}=u\right)
$$

where

$$
\mathcal{H}_{Y_{1}^{\beta}}\left(\nabla_{\beta} Y_{1}^{\beta}\right):=\left(\begin{array}{l}
\mathcal{H}_{Y_{1}^{\beta}}\left(\partial_{\theta} Y_{1}^{\beta}\right. \\
\mathcal{H}_{Y_{1}^{\beta}}\left(\partial_{\sigma} Y_{1}^{\beta}\right.
\end{array}\right)=-2\left(\begin{array}{c}
\partial_{\theta} Y_{1}^{\beta} \\
\partial_{\sigma} Y_{1}^{\beta}
\end{array}\right) \frac{L Y_{1}^{\beta}}{U_{1}^{\beta}}+\left(\begin{array}{c}
\partial_{\theta} Y_{1}^{\beta} \\
\partial_{\sigma} Y_{1}^{\beta}
\end{array}\right) \frac{W_{1}^{\beta}}{\left(U_{1}^{\beta}\right)^{2}}-\frac{1}{U_{1}^{\beta}}\left(\begin{array}{c}
\Gamma\left(Y_{1}^{\beta}, \partial_{\theta} Y_{1}^{\beta}\right) \\
\Gamma\left(Y_{1}^{\beta}, \partial_{\sigma} Y_{1}^{\beta}\right)
\end{array}\right),
$$

$L Y_{1}^{\beta}, U_{1}^{\beta}$ and $W_{1}^{\beta}$ are given in Theorem A.2, the process $\left(V_{t}^{\theta}\right)=\Gamma\left(Y_{t}^{\beta}, \partial_{\theta} Y_{t}^{\beta}\right)$ is the solution of

$$
V_{t}^{\theta}=2 \int_{0}^{t} a^{\prime}\left(Y_{s}^{\beta}, \theta\right) V_{s}^{\theta} \mathrm{d} s+\int_{0}^{t} U_{s}^{\beta}\left[\left(\partial_{\theta} a\right)^{\prime}\left(Y_{s}^{\beta}, \theta\right)+a^{\prime \prime}\left(Y_{s}^{\beta}, \theta\right) \partial_{\theta} Y_{s}^{\beta}\right] \mathrm{d} s,
$$


and the process $\left(V_{t}^{\sigma}\right)=\Gamma\left(Y_{t}^{\beta}, \partial_{\sigma} Y_{t}^{\beta}\right)$ is the solution of

$$
V_{t}^{\sigma}=2 \int_{0}^{t} a^{\prime}\left(Y_{s}^{\beta}, \theta\right) V_{s}^{\sigma} \mathrm{d} s+\int_{0}^{t} a^{\prime \prime}\left(Y_{s}^{\beta}, \theta\right) \partial_{\sigma} Y_{s}^{\beta} U_{s}^{\beta} \mathrm{d} s+c^{2} \sigma \int_{0}^{t} \int_{E} \rho(z) \mu(\mathrm{d} s, \mathrm{~d} z) .
$$

Proof. Theorem A.3 is an extension of Theorem 5 in [5] where the main novelty is the expression for $\frac{\partial_{\sigma} q^{\beta}}{q^{\beta}}$. For the computation of the new term $\mathcal{H}_{Y_{1}^{\beta}}\left(\partial_{\sigma} Y_{1}^{\beta}\right)$, we apply Theorem 10-3 in [2] to the stochastic differential equation satisfied by the vector $\left(Y_{t}^{\beta}, U_{t}^{\beta}, \partial_{\sigma} Y_{t}^{\beta}\right)^{T}$, this gives the above expression for $\left(V_{t}^{\sigma}\right)$.

We end this subsection with an explicit representation of $\nabla_{\beta} q^{\beta}(u)$ which gives a computation of the iterated Malliavin weight $\mathcal{H}_{Y_{1}^{\beta}}\left(\mathcal{H}_{Y_{1}^{\beta}}\left(\nabla_{\beta} Y_{1}^{\beta}\right)\right)$.

Theorem A.4. Under the assumptions of Theorem A.2,

$$
\nabla_{\beta} q^{\beta}(u)=\left(\begin{array}{c}
\partial_{\theta} q^{\beta}(u) \\
\partial_{\sigma} q^{\beta}(u)
\end{array}\right)=\mathbb{E}\left[1_{\left\{Y_{1}^{\beta} \geq u\right\}} \mathcal{H}_{Y_{1}^{\beta}}\left(\mathcal{H}_{Y_{1}^{\beta}}\left(\nabla_{\beta} Y_{1}^{\beta}\right)\right)\right]
$$

where

$$
\mathcal{H}_{Y_{1}^{\beta}}\left(\mathcal{H}_{Y_{1}^{\beta}}\left(\nabla_{\beta} Y_{1}^{\beta}\right)\right)=-2 \mathcal{H}_{Y_{1}^{\beta}}\left(\nabla_{\beta} Y_{1}^{\beta}\right) \frac{L Y_{1}^{\beta}}{U_{1}^{\beta}}+\mathcal{H}_{Y_{1}^{\beta}}\left(\nabla_{\beta} Y_{1}^{\beta}\right) \frac{W_{1}^{\beta}}{\left(U_{1}^{\beta}\right)^{2}}-\left(\begin{array}{c}
\Gamma\left(Y_{1}^{\beta}, \mathcal{H}_{Y_{1}^{\beta}}\left(\partial_{\theta} Y_{1}^{\beta}\right)\right) \\
\Gamma\left(Y_{1}^{\beta}, \mathcal{H}_{Y_{1}^{\beta}}\left(\partial_{\sigma} Y_{1}^{\beta}\right)\right)
\end{array}\right) \frac{1}{U_{1}^{\beta}},
$$

where $\partial_{\theta} Y_{1}^{\beta}, \partial_{\sigma} Y_{1}^{\beta}$ are respectively given by equations (A.11), (A.12) and $U_{1}^{\beta}, W_{1}^{\beta}$ are computed in Theorem A.2, $\mathcal{H}_{Y_{1}^{\beta}}\left(\nabla_{\beta} Y_{1}^{\beta}\right)$ is given in Theorem A.3.

Proof. Let $f$ be a smooth functions with compact support. Then,

$$
\nabla_{\beta} \mathbb{E}\left[f\left(Y_{1}^{\beta}\right)\right]=\int_{\mathbb{R}} \mathrm{d} u \nabla_{\beta} q^{\beta}(u) f(u)
$$

On the other hand, using the integration by parts formula of the Malliavin calculus, we have

$$
\begin{aligned}
\nabla_{\beta} \mathbb{E}\left[f\left(Y_{1}^{\beta}\right)\right] & =\mathbb{E}\left[f^{\prime}\left(Y_{1}^{\beta}\right) \nabla_{\beta} Y_{1}^{\beta}\right] \\
& =\mathbb{E}\left[f\left(Y_{1}^{\beta}\right) \mathcal{H}_{Y_{1}^{\beta}}\left(\nabla_{\beta} Y_{1}^{\beta}\right)\right] \\
& =\mathbb{E}\left[F\left(Y_{1}^{\beta}\right) \mathcal{H}_{Y_{1}^{\beta}}\left(\mathcal{H}_{Y_{1}^{\beta}}\left(\nabla_{\beta} Y_{1}^{\beta}\right)\right)\right],
\end{aligned}
$$

where $F$ denotes a primitive function of $f$. If $f$ converges to Dirac mass at some point $u$, from the estimates above, we can deduce (A.17). Moreover, from (A.5) we also get (A.18).

To complete the result of Theorem A.4, we give the expressions for $\Gamma\left(Y_{1}^{\beta}, \mathcal{H}_{Y_{1}^{\beta}}\left(\partial_{\theta} Y_{1}^{\beta}\right)\right)$ and $\Gamma\left(Y_{1}^{\beta}, \mathcal{H}_{Y_{1}^{\beta}}\left(\partial_{\sigma} Y_{1}^{\beta}\right)\right)$. 
Lemma A.5. Under the assumptions of Theorem A.2,

$$
\begin{aligned}
\left(\begin{array}{c}
\Gamma\left(Y_{1}^{\beta}, \mathcal{H}_{Y_{1}^{\beta}}\left(\partial_{\theta} Y_{1}^{\beta}\right)\right) \\
\Gamma\left(Y_{1}^{\beta}, \mathcal{H}_{Y_{1}^{\beta}}\left(\partial_{\sigma} Y_{1}^{\beta}\right)\right)
\end{array}\right)= & \left(\begin{array}{c}
V_{1}^{\theta} \\
V_{1}^{\sigma}
\end{array}\right) \mathcal{H}_{Y_{1}^{\beta}}(1)-\left(\begin{array}{c}
\partial_{\theta} Y_{1}^{\beta} \\
\partial_{\sigma} Y_{1}^{\beta}
\end{array}\right) \frac{2 D_{1}^{\beta}}{U_{1}^{\beta}}-\left(\begin{array}{c}
\partial_{\theta} Y_{1}^{\beta} \\
\partial_{\sigma} Y_{1}^{\beta}
\end{array}\right) \frac{\mathcal{H}_{Y_{1}^{\beta}}(1) W_{1}^{\beta}}{U_{1}^{\beta}}+\left(\begin{array}{c}
\partial_{\theta} Y_{1}^{\beta} \\
\partial_{\sigma} Y_{1}^{\beta}
\end{array}\right) \frac{Q_{1}^{\beta}}{\left(U_{1}^{\beta}\right)^{2}} \\
& -\left(\begin{array}{c}
T_{1}^{\theta} \\
T_{1}^{\sigma}
\end{array}\right) \frac{1}{U_{1}^{\beta}}+\left(\begin{array}{c}
V_{1}^{\theta} \\
V_{1}^{\sigma}
\end{array}\right) \frac{W_{1}^{\beta}}{\left(U_{1}^{\beta}\right)^{2}}
\end{aligned}
$$

where $\partial_{\theta} Y_{1}^{\beta}, \partial_{\sigma} Y_{1}^{\beta}$ are respectively given in (A.11), (A.12), $U_{1}^{\beta}, W_{1}^{\beta}$ are computed in Theorem A.2, $V_{1}^{\theta}, V_{1}^{\sigma}$ are computed in Theorem A.3, $\mathcal{H}_{Y_{1}^{\beta}}(1)$ is given in (A.7) and $D_{1}^{\beta}=\Gamma\left(Y_{1}^{\beta}, L Y_{1}^{\beta}\right), Q_{1}^{\beta}=\Gamma\left(Y_{1}^{\beta}, W_{1}^{\beta}\right), T_{1}^{\theta}=$ $\Gamma\left(Y_{1}^{\beta}, V_{1}^{\theta}\right)$ and $T_{1}^{\sigma}=\Gamma\left(Y_{1}^{\beta}, V_{1}^{\sigma}\right)$.

Proof. From the basic properties of the operators $L$ and $\Gamma$ (linearity and the chain rule property) stated in Section A.1, we get that

$$
\begin{aligned}
& \Gamma\left(Y_{1}^{\beta}, \mathcal{H}_{Y_{1}^{\beta}}\left(\partial_{\theta} Y_{1}^{\beta}\right)\right)=\Gamma\left[Y_{1}^{\beta},-2 \partial_{\theta} Y_{1}^{\beta} \frac{L Y_{1}^{\beta}}{U_{1}^{\beta}}\right]+\Gamma\left[Y_{1}^{\beta}, \partial_{\theta} Y_{1}^{\beta} \frac{W_{1}^{\beta}}{\left(U_{1}^{\beta}\right)^{2}}\right]+\Gamma\left[Y_{1}^{\beta},-\frac{\Gamma\left(Y_{1}^{\beta}, \partial_{\theta} Y_{1}^{\beta}\right)}{U_{1}^{\beta}}\right], \\
& \Gamma\left(Y_{1}^{\beta}, \mathcal{H}_{Y_{1}^{\beta}}\left(\partial_{\sigma} Y_{1}^{\beta}\right)\right)=\Gamma\left[Y_{1}^{\beta},-2 \partial_{\sigma} Y_{1}^{\beta} \frac{L Y_{1}^{\beta}}{U_{1}^{\beta}}\right]+\Gamma\left[Y_{1}^{\beta}, \partial_{\sigma} Y_{1}^{\beta} \frac{W_{1}^{\beta}}{\left(U_{1}^{\beta}\right)^{2}}\right]+\Gamma\left[Y_{1}^{\beta},-\frac{\Gamma\left(Y_{1}^{\beta}, \partial_{\sigma} Y_{1}^{\beta}\right)}{U_{1}^{\beta}}\right],
\end{aligned}
$$

where

$$
\begin{gathered}
\Gamma\left[Y_{1}^{\beta},-2 \partial_{\theta} Y_{1}^{\beta} \frac{L Y_{1}^{\beta}}{U_{1}^{\beta}}\right]=-2 \frac{L Y_{1}^{\beta}}{U_{1}^{\beta}} \Gamma\left(Y_{1}^{\beta}, \partial_{\theta} Y_{1}^{\beta}\right)-2 \frac{\partial_{\theta} Y_{1}^{\beta}}{U_{1}^{\beta}} \Gamma\left(Y_{1}^{\beta}, L Y_{1}^{\beta}\right)+2 \partial_{\theta} Y_{1}^{\beta} \frac{L Y_{1}^{\beta}}{\left(U_{1}^{\beta}\right)^{2}} \Gamma\left(Y_{1}^{\beta}, U_{1}^{\beta}\right) \\
=-2 \frac{L Y_{1}^{\beta}}{U_{1}^{\beta}} V_{1}^{\theta}-2 \frac{\partial_{\theta} Y_{1}^{\beta}}{U_{1}^{\beta}} D_{1}^{\beta}+2 \partial_{\theta} Y_{1}^{\beta} \frac{L Y_{1}^{\beta}}{\left(U_{1}^{\beta}\right)^{2}} W_{1}^{\beta} . \\
\Gamma\left[Y_{1}^{\beta}, \partial_{\theta} Y_{1}^{\beta} \frac{W_{1}^{\beta}}{\left(U_{1}^{\beta}\right)^{2}}\right]=\frac{W_{1}^{\beta}}{\left(U_{1}^{\beta}\right)^{2}} \Gamma\left(Y_{1}^{\beta}, \partial_{\theta} Y_{1}^{\beta}\right)+\frac{\partial_{\theta} Y_{1}^{\beta}}{\left(U_{1}^{\beta}\right)^{2}} \Gamma\left(Y_{1}^{\beta}, W_{1}^{\beta}\right)-\frac{2 \partial_{\theta} Y_{1}^{\beta} W_{1}^{\beta}}{\left(U_{1}^{\beta}\right)^{3}} \Gamma\left(Y_{1}^{\beta}, U_{1}^{\beta}\right) \\
=\frac{W_{1}^{\beta}}{\left(U_{1}^{\beta}\right)^{2}} V_{1}^{\theta}+\frac{\partial_{\theta} Y_{1}^{\beta}}{\left(U_{1}^{\beta}\right)^{2}} Q_{1}^{\beta}-\frac{2 \partial_{\theta} Y_{1}^{\beta} W_{1}^{\beta}}{\left(U_{1}^{\beta}\right)^{3}} W_{1}^{\beta} . \\
\Gamma\left[Y_{1}^{\beta},-\frac{\Gamma\left(Y_{1}^{\beta}, \partial_{\theta} Y_{1}^{\beta}\right)}{U_{1}^{\beta}}\right]=-\frac{\Gamma\left(Y_{1}^{\beta}, \Gamma\left(Y_{1}^{\beta}, \partial_{\theta} Y_{1}^{\beta}\right)\right)}{U_{1}^{\beta}}+\frac{\Gamma\left(Y_{1}^{\beta}, \partial_{\theta} Y_{1}^{\beta}\right)}{\left(U_{1}^{\beta}\right)^{2}} \Gamma\left(Y_{1}^{\beta}, U_{1}^{\beta}\right)=-\frac{T_{1}^{\theta}}{U_{1}^{\beta}}+\frac{V_{1}^{\theta}}{\left(U_{1}^{\beta}\right)^{2}} W_{1}^{\beta} .
\end{gathered}
$$


Similarly, we have

$$
\begin{aligned}
\Gamma\left[Y_{1}^{\beta},-2 \partial_{\sigma} Y_{1}^{\beta} \frac{L Y_{1}^{\beta}}{U_{1}^{\beta}}\right] & =-2 \frac{L Y_{1}^{\beta}}{U_{1}^{\beta}} V_{1}^{\sigma}-2 \frac{\partial_{\sigma} Y_{1}^{\beta}}{U_{1}^{\beta}} D_{1}^{\beta}+2 \partial_{\sigma} Y_{1}^{\beta} \frac{L Y_{1}^{\beta}}{\left(U_{1}^{\beta}\right)^{2}} W_{1}^{\beta} . \\
\Gamma\left[Y_{1}^{\beta}, \partial_{\sigma} Y_{1}^{\beta} \frac{W_{1}^{\beta}}{\left(U_{1}^{\beta}\right)^{2}}\right] & =\frac{W_{1}^{\beta}}{\left(U_{1}^{\beta}\right)^{2}} V_{1}^{\sigma}+\frac{\partial_{\sigma} Y_{1}^{\beta}}{\left(U_{1}^{\beta}\right)^{2}} Q_{1}^{\beta}-\frac{2 \partial_{\sigma} Y_{1}^{\beta} W_{1}^{\beta}}{\left(U_{1}^{\beta}\right)^{3}} W_{1}^{\beta} . \\
\Gamma\left[Y_{1}^{\beta},-\frac{\Gamma\left(Y_{1}^{\beta}, \partial_{\sigma} Y_{1}^{\beta}\right)}{U_{1}^{\beta}}\right] & =-\frac{T_{1}^{\sigma}}{U_{1}^{\beta}}+\frac{V_{1}^{\sigma}}{\left(U_{1}^{\beta}\right)^{2}} W_{1}^{\beta} .
\end{aligned}
$$

Then, from (A.7) and the above estimates, we get the formula (A.19), after some calculus and the proof is complete.

Lemma A.6. Under the assumptions of Theorem A.2, there are versions of the processes $\left(D_{t}^{\beta}\right)=$ $\left(\Gamma\left(Y_{t}^{\beta}, L Y_{t}^{\beta}\right)\right)_{t},\left(Q_{t}^{\beta}\right)=\Gamma\left(Y_{t}^{\beta}, W_{t}^{\beta}\right)_{t},\left(T_{t}^{\theta}\right)_{t}=\left(\Gamma\left(Y_{t}^{\beta}, V_{t}^{\theta}\right)\right)_{t}$ and $\left(T_{t}^{\sigma}\right)_{t}=\left(\Gamma\left(Y_{t}^{\beta}, V_{t}^{\sigma}\right)\right)_{t}$ that are solutions of the linear equations:

$$
\begin{aligned}
D_{t}^{\beta}= & 2 \int_{0}^{t} a^{\prime}\left(Y_{s}^{\beta}, \theta\right) D_{s}^{\beta} \mathrm{d} s+\int_{0}^{t} a^{\prime \prime}\left(Y_{s}^{\beta}, \theta\right) L Y_{s}^{\beta} U_{s}^{\beta} \mathrm{d} s+\frac{1}{2} \int_{0}^{t} a^{\prime \prime}\left(Y_{s}^{\beta}, \theta\right) W_{s}^{\beta} \mathrm{d} s+\frac{1}{2} \int_{0}^{t} a^{\prime \prime \prime}\left(Y_{s}^{\beta}, \theta\right)\left(U_{s}^{\beta}\right)^{2} \mathrm{~d} s \\
& +\frac{c^{2} \sigma^{2}}{2} \int_{0}^{t} \int_{E} \rho(z)\left(\rho^{\prime}(z)+\rho(z) \frac{g^{\prime}(z)}{g(z)}\right)^{\prime} \mu(\mathrm{d} s, \mathrm{~d} z) \\
Q_{t}^{\beta}= & 4 \int_{0}^{t} a^{\prime}\left(Y_{s}^{\beta}, \theta\right) Q_{s}^{\beta} \mathrm{d} s+7 \int_{0}^{t} a^{\prime \prime}\left(Y_{s}^{\beta}, \theta\right) W_{s}^{\beta} U_{s}^{\beta} \mathrm{d} s+2 \int_{0}^{t} a^{\prime \prime \prime}\left(Y_{s}^{\beta}, \theta\right)\left(U_{s}^{\beta}\right)^{3} \mathrm{~d} s \\
& +c^{4} \sigma^{4} \int_{0}^{t} \int_{E} \rho(z)\left[\left(\rho(z)^{\prime}\right)^{2}+\rho(z) \rho(z)^{\prime \prime}\right] \mu(\mathrm{d} s, \mathrm{~d} z) \\
T_{t}^{\theta}= & 3 \int_{0}^{t} a^{\prime}\left(Y_{s}^{\beta}, \theta\right) T_{s}^{\theta} \mathrm{d} s+3 \int_{0}^{t} a^{\prime \prime}\left(Y_{s}^{\beta}, \theta\right) V_{s}^{\theta} U_{s}^{\beta} \mathrm{d} s+\int_{0}^{t}\left(\partial_{\theta} a\right)^{\prime}\left(Y_{s}^{\beta}, \theta\right) W_{s}^{\beta} \mathrm{d} s+\int_{0}^{t} a^{\prime \prime}\left(Y_{s}^{\beta}, \theta\right) \partial_{\theta} Y_{s}^{\beta} W_{s}^{\beta} \mathrm{d} s \\
& +\int_{0}^{t}\left(\partial_{\theta} a\right)^{\prime \prime}\left(Y_{s}^{\beta}, \theta\right)\left(U_{s}^{\beta}\right)^{2}+\int_{0}^{t} a^{\prime \prime \prime}\left(Y_{s}^{\beta}, \theta\right) \partial_{\theta} Y_{s}^{\beta}\left(U_{s}^{\beta}\right)^{2} \mathrm{~d} s, \\
T_{t}^{\sigma}= & 3 \int_{0}^{t} a^{\prime}\left(Y_{s}^{\beta}, \theta\right) T_{s}^{\beta} \mathrm{d} s+3 \int_{0}^{t} a^{\prime \prime}\left(Y_{s}^{\beta}, \theta\right) V_{s}^{\sigma} U_{s}^{\beta} \mathrm{d} s+\int_{0}^{t} a^{\prime \prime}\left(Y_{s}^{\beta}, \theta\right) \partial_{\sigma} Y_{s}^{\beta} W_{s}^{\beta} \mathrm{d} s \\
& +\int_{0}^{t} a^{\prime \prime \prime}\left(Y_{s}^{\beta}, \theta\right) \partial_{\sigma} Y_{s}^{\beta}\left(U_{s}^{\beta}\right)^{2} \mathrm{~d} s+c^{3} \sigma^{2} \int_{0}^{t} \int_{E} \rho(z) \rho(z)^{\prime} \mu(\mathrm{d} s, \mathrm{~d} z) .
\end{aligned}
$$

Proof. The proof of Lemma A.6 is a direct consequence of Theorem 10-3 in [2]. Indeed, considering the stochastic differential equation satisfied by the vector $\left(Y_{t}^{\beta}, L Y_{t}^{\beta}, U_{t}^{\beta}, W_{t}^{\beta}, V_{t}^{\theta}, V_{t}^{\sigma}, \partial_{\theta} Y_{t}^{\beta}, \partial_{\sigma} Y_{t}^{\beta}\right)^{T}$ and using Theorem 10-3 in [2], we prove that the processes $\left(D_{t}^{\beta}\right)=\left(\Gamma\left(Y_{t}^{\beta}, L Y_{t}^{\beta}\right)\right)_{t},\left(Q_{t}^{\beta}\right)=\Gamma\left(Y_{t}^{\beta}, W_{t}^{\beta}\right)_{t},\left(T_{t}^{\theta}\right)_{t}=\left(\Gamma\left(Y_{t}^{\beta}, V_{t}^{\theta}\right)\right)_{t}$ and $\left(T_{t}^{\sigma}\right)_{t}=\left(\Gamma\left(Y_{t}^{\beta}, V_{t}^{\sigma}\right)\right)_{t}$ are solutions of linear equations, respectively, given by (A.20)-(A.23). 


\section{REFERENCES}

[1] D. Applebaum, Lévy Processes and Stochastic Calculus, 2nd edn. Vol. 116 of Cambridge Studies in Advanced Mathematics. Cambridge University Press, Cambridge (2009).

[2] K. Bichteler, J.-B. Gravereaux and J. Jacod, Malliavin Calculus for Processes with Jumps. Vol. 2 of Stochastics Monographs. Gordon and Breach Science Publishers, New York (1987).

[3] N. Bouleau and L. Denis, Dirichlet Forms Methods for Poisson Point Measures and Lévy Processes. Vol. 76 of Probability Theory and Stochastic Modelling. Springer, Cham (2015). With emphasis on the creation-annihilation techniques.

[4] E. Çınlar, Probability and Stochastics. Vol. 261 of Graduate Texts in Mathematics. Springer, New York (2011).

[5] E. Clément and A. Gloter, Local asymptotic mixed normality property for discretely observed stochastic differential equations driven by stable Lévy processes. Stochastic Process. Appl. 125 (2015) 2316-2352.

[6] E. Clément, A. Gloter and H. Nguyen, LAMN property for the drift and volatility parameters of a SDE driven by a stable Lévy process. Preprint hal-01472749v2 (2017).

[7] A. Debussche and N. Fournier, Existence of densities for stable-like driven SDE's with Hölder continuous coefficients. J. Funct. Anal. 264 (2013) 1757-1778.

[8] L. Denis, A criterion of density for solutions of Poisson-driven SDEs. Probab. Theory Related Fields 118 (2000) $406-426$.

[9] N. Fournier and J. Printems, Absolute continuity for some one-dimensional processes. Bernoulli 16 (2010) 343-360.

[10] Y. Ishikawa and H. Kunita, Malliavin calculus on the Wiener-Poisson space and its application to canonical SDE with jumps. Stochastic Process. Appl. 116 (2006) 1743-1769.

[11] D. Ivanenko and A. Kulik, Malliavin calculus approach to statistical inference for Lévy driven SDE's. Methodol. Comput. Appl. Probab. 17 (2015) 107-123.

[12] P. Jeganathan, On the asymptotic theory of estimation when the limit of the log-likelihood ratios is mixed normal. Sankhy 44 (1982) 173-212.

[13] A. Kulik, On weak uniqueness and distributional properties of a solution to an SDE with $\alpha$-stable noise. Preprint arXiv: 1511.00106.v1 (2015).

[14] J. Picard, On the existence of smooth densities for jump processes. Probab. Theory Related Fields 105 (1996) $481-511$. 